\title{
Cubic interaction vertices for massive and massless higher spin fields
}

\author{
R.R. Metsaev* \\ Department of Theoretical Physics, P.N. Lebedev Physical Institute, \\ Leninsky prospect 53, Moscow 119991, Russia
}

\begin{abstract}
Using the light-cone formulation of relativistic dynamics, we develop various methods for constructing cubic interaction vertices and apply these methods to the study of higher spin fields propagating in flat space of dimension greater than or equal to four. Generating functions of parity invariant cubic interaction vertices for massive and massless higher spin fields of arbitrary symmetry are obtained. We derive restrictions on the allowed values of spins and the number of derivatives, which provide a classification of cubic interaction vertices for totally symmetric fields. As an example of application of the light-cone formalism, we obtain simple expressions for the minimal Yang-Mills and gravitational interactions of massive totally symmetric arbitrary spin fields. We give the complete list of parity invariant and parity violating cubic interaction vertices that can be constructed for massless fields in five and six-dimensional spaces.
\end{abstract}

Keywords: Light-cone formalism, interaction vertices, higher spin fields.

PACS-2006: 11.10.-z; 11.30-j; 11.30.Cp

\footnotetext{
*E-mail: metsaev@lpi.ru
} 


\section{Introduction}

The light-cone formalism [1]-[4] offers conceptual and technical simplifications of approaches to various problems of modern quantum field and string theories. This formalism hides some of the symmetries and makes the notation somewhat cumbersome but eventually turns out to be rather effective. A number of important problems have been solved in the framework of this formalism. For example, we mention the solution to the light-cone gauge string field theory [5][9] and the construction of a superfield formulation for some versions of supersymmetric theories [10]-[14]. Theories formulated within this formalism may sometimes be a good starting point for deriving a Lorentz covariant formulation [15]-[19]. Another attractive application of the lightcone formalism is the construction of interaction vertices in the theory of massless higher spin fields [20]-[23]. Some interesting applications of the light-cone formalism to field theory such as QCD are reviewed in [24]. Discussions of super $p$-branes and string bit models in the light-cone gauge is given in [25, 26] and [27] respectively.

In this paper, we apply the light-cone formalism to study interaction vertices for higher spin fields. Considerable progress has been achieved in the problem of constructing the theory describing the interaction of massless higher spin fields with gravity. In Ref.[28], cubic interaction vertices for massless higher spin fields propagating in $A d S_{4}$ space were constructed; in Ref.[29], nonlinear equations of motion to all orders in the coupling constant for massless higher spin fields in $A d S_{4}$ were found. Nonlinear equations of motion for massless totally symmetric higher spin fields in $A d S_{d}$ space ( $d \geq 4$ ) were found in Ref.[30] (see [31],[32] for a recent review). It now becomes apparent that constructing a self-consistent theory of massless higher spin fields interacting with gravity requires formulating the theory in $A d S$ space. Unfortunately, despite the efforts, an action that leads to the above-mentioned nonlinear equations of motion has not yet been obtained. To quantize these theories and investigate their ultraviolet behavior, it would be important to find an appropriate action. Since the massless higher spin field theories correspond quantum mechanically to non-local point particles in a space of certain auxiliary variables, it is conjectured that such theories may be ultraviolet finite [33]. We believe that the light-cone formulation may be helpful in understanding these theories better. The situation here may be analogous to that in string theory; a covariant formulation of the closed string field theories is non-polynomial and is not useful for practical calculations, while the light-cone formulation restricts the string action to the cubic order in string fields.

In this paper, keeping these extremely important applications in mind, we develop various methods for constructing cubic interaction vertices and use these methods to find cubic vertices for massive and massless arbitrary spin fields propagating in flat space. We believe that most of our approach to massless higher spin fields can be relatively straightforwardly generalized to the case of massless higher spin fields in $A d S$ space. The light-cone gauge approach to dynamics of free fields in $A d S$ space was developed in [34] (see also [35],[36]). Although the light-cone approach in $A d S$ space is complicated compared to that in flat space, it turns out that these approaches share many properties. We therefore believe that methods developed in flat space might be helpful in analyzing dynamics of interacting massless higher spin fields in $A d S$ space. As regards our study of massive fields, we note that our interest in light-cone gauge vertices for massive higher spin fields in flat space is motivated, among other things, by the potential of our approach for in-depth studies of the interaction vertices of the light-cone gauge (super)string field theory.

At present, a wide class of cubic interaction vertices for fields propagating in flat space is known. In particular, the self-interaction cubic vertices for the massless spin 3 field were found in [37]-[40] and the higher-derivative cubic vertex for massless spin 2 and spin 4 fields was studied 
in [41]. More general examples of the cubic interaction vertices for massless higher spin fields were discovered in [20, 21, 42] and the full list of cubic interaction vertices for massless higher spin fields was given in [22]. A wide list of cubic interaction vertices for massive higher spin fields was obtained in [43] (see also [44],[45]). With the exception of Refs.[39, 40] (devoted to spin 3 field self-interactions) all the above-mentioned works were devoted to the analysis of interaction vertices for higher spin fields in $4 d$ flat space. In view of possible applications of the higher spin field theory to string theory, it is instructive to study cubic interaction vertices for higher spin fields in space of dimension $d \geq 4$. We do this in the present paper.

This paper is organized as follows. In Section 2, we introduce the notation and describe the standard manifestly $s o(d-2)$ covariant light-cone formulation of free massless and massive fields.

In Section 3, we discuss arbitrary $n$-point interaction vertices and find restrictions imposed by kinematical symmetries of the Poincaré algebra on these vertices.

In Section 4, we study restrictions imposed by kinematical and dynamical symmetries of the Poincaré algebra on cubic interaction vertices for massless and massive fields. We find various forms of closed equations on cubic interaction vertices.

In Section 5, we present solution to equations for parity invariant cubic interaction vertices of massless fields. Section 6 is devoted to parity invariant cubic interaction vertices for massless and massive fields. We apply our general results to derive the minimal Yang-Mills and gravitational interactions of massive arbitrary spin fields. Our approach allows us to obtain simple expressions for vertices of these interactions. In Section 7, we discuss parity invariant cubic interaction vertices for massive fields. In Sections 5,7, we also derive restrictions on the allowed values of spins and the number of derivatives for cubic interaction vertices of the totally symmetric fields.

In Section 8, we develop the light-cone formalism with manifestly realized $s o(d-4)$ symmetries that allows us to study both the parity invariant and parity violating interaction vertices on an equal footing. To illustrate this formalism, we construct cubic interaction vertices for massless totally symmetric fields in $5 d$ space and for massless totally symmetric and mixed-symmetry fields in $6 d$ space. We present the complete list of cubic interaction vertices that can be constructed for massless fields in $d=5,6$ dimensions.

Section 9 summarizes our conclusions and suggests directions for future research. Appendices contain some mathematical details and useful formulas.

\section{Free light-cone gauge massive and massless fields}

The method suggested in Ref.[1] reduces the problem of finding a new (light-cone gauge) dynamical system to the problem of finding a new solution of defining symmetry algebra commutators 1 . Since in our case the defining symmetries are generated by the Poincaré algebra, we begin with a discussion of the realization of the Poincaré algebra on the space of massive and massless fields. We focus on free fields in this section.

The Poincaré algebra of $d$-dimensional Minkowski space is spanned by translation generators $P^{A}$ and rotation generators $J^{A B}$ (the latter span the $s o(d-1,1)$ Lorentz algebra). The Lorentz covariant form of the non-trivial Poincaré algebra commutators is

$$
\left[P^{A}, J^{B C}\right]=\eta^{A B} P^{C}-\eta^{A C} P^{B}, \quad\left[J^{A B}, J^{C D}\right]=\eta^{B C} J^{A D}+3 \text { terms }
$$

${ }^{1}$ This method is the Hamiltonian version of the Noether method for finding a new dynamical system. An interesting up-to date discussion of the Noether method may be found in [46]. 
where $\eta^{A B}$ stands for the mostly positive flat metric tensor. The generators $P^{A}$ are chosen to be hermitian, and the $J^{A B}$ to be antihermitian. To develop the light-cone formulation, in place of the Lorentz basis coordinates $x^{A}$ we introduce the light-cone basis coordinates $x^{ \pm}, x^{I}$ defined by 2

$$
x^{ \pm} \equiv \frac{1}{\sqrt{2}}\left(x^{d-1} \pm x^{0}\right), \quad x^{I}, \quad I=1, \ldots, d-2,
$$

and treat $x^{+}$as an evolution parameter. In this notation, the Lorentz basis vector $X^{A}$ is decomposed as $\left(X^{+}, X^{-}, X^{I}\right)$ and a scalar product of two vectors is then decomposed as

$$
\eta_{A B} X^{A} Y^{B}=X^{+} Y^{-}+X^{-} Y^{+}+X^{I} Y^{I}
$$

where the covariant and contravariant components of vectors are related as $X^{+}=X_{-}, X^{-}=X_{+}$, $X^{I}=X_{I}$. Here and henceforth, a summation over repeated transverse indices is understood. In the light-cone formalism, the Poincaré algebra generators can be separated into two groups:

$$
\begin{array}{lllll}
P^{+}, \quad P^{I}, \quad J^{+I}, \quad J^{+-}, & J^{I J}, & \text { kinematical generators } ; \\
P^{-}, \quad J^{-I}, & & \text { dynamical generators } .
\end{array}
$$

For $x^{+}=0$, the kinematical generators in the field realization are quadratic in the physical fields 3 , while the dynamical generators receive higher-order interaction-dependent corrections.

Commutators of the Poincaré algebra in light-cone basis can be obtained from (2.1) by using the light-cone metric having the following non vanishing elements: $\eta^{+-}=\eta^{-+}=1, \eta^{I J}=\delta^{I J}$. Hermitian conjugation rules of the Poincaré algebra generators in light-cone basis take the form

$$
P^{ \pm \dagger}=P^{ \pm}, \quad P^{I \dagger}=P^{I}, \quad J^{I J \dagger}=-J^{I J}, \quad J^{+-\dagger}=-J^{+-}, \quad J^{ \pm I \dagger}=-J^{ \pm I} .
$$

To find a realization of the Poincaré algebra on the space of massive and massless fields we use the light-cone gauge description of those fields. We discuss massive and massless fields in turn.

Mixed-symmetry massive fields. In order to obtain the light-cone gauge description of a massive mixed-symmetry field in an easy-to-use form, let us introduce a finite set of the creation and annihilation operators $\alpha_{n}^{I}, \alpha_{n}$ and $\bar{\alpha}_{n}^{I}, \bar{\alpha}_{n}(n=1,2, \ldots, \nu)$ defined by the relations

$$
\begin{gathered}
{\left[\bar{\alpha}_{n}^{I}, \alpha_{m}^{J}\right]=\delta_{n m} \delta^{I J}, \quad\left[\bar{\alpha}_{n}, \alpha_{m}\right]=\delta_{n m},} \\
\bar{\alpha}_{n}^{I}|0\rangle=0, \quad \bar{\alpha}_{n}|0\rangle=0 .
\end{gathered}
$$

The oscillators $\alpha_{n}^{I}, \bar{\alpha}_{n}^{I}$ and $\alpha_{n}, \bar{\alpha}_{n}$ transform in the respective vector and scalar representations of the $s o(d-2)$ algebra. In $d$-dimensional Minkowski space, the massive arbitrary spin field is labeled by the mass parameter $\mathrm{m}$ and spin labels $\left.s_{1}, \ldots, s_{\nu}, \nu=\left[\frac{d-1}{2}\right]\right]^{4}$. Physical D.o.F of the massive field labeled by spin labels $s_{1}, \ldots, s_{\nu}$ can be collected into a ket-vector defined by

$$
\left|\phi_{s_{1} \ldots s_{\nu}}(p, \alpha)\right\rangle \equiv \prod_{n=1}^{\nu} \sum_{t_{n}=0}^{s_{n}} \alpha_{n}^{I_{1}^{n}} \ldots \alpha_{n}^{I_{s_{n}-t_{n}}^{n}} \alpha_{n}^{t_{n}} \phi_{s_{1} \ldots s_{\nu}}^{I_{1}^{1} \ldots I_{s_{1}-t_{1}}^{1} \ldots I_{1}^{\nu} \ldots I_{s_{\nu}-t_{\nu}}^{\nu}}(p)|0\rangle .
$$

\footnotetext{
${ }^{2} A, B, C, D=0,1, \ldots, d-1$ are $s o(d-1,1)$ vector indices; 'transverse' indices $I, J, K=1, \ldots, d-2$ are so $(d-2)$ vector indices; $i, j=1, \ldots, d-4$ are $s o(d-4)$ vector indices.

${ }^{3}$ Namely, for $x^{+} \neq 0$ they have a structure $G=G_{1}+x^{+} G_{2}$, where $G_{1}$ is quadratic in fields, while $G_{2}$ contains higher order terms in fields.

${ }^{4}$ In $4 d$ flat space, massive arbitrary spin field is labeled by the mass parameter $\mathrm{m}$ and by one spin label $s=s_{1}$. Appearance of the spin labels $s_{1}, \ldots, s_{\nu}$ is related to the fact that physical spin D.o.F of massive field in $d$-dimensional flat space are described by the $s o(d-1)$ algebra irreps labeled by $\left[\frac{d-1}{2}\right]$ Gelfand-Zetlin (or Dynkin) labels.
} 
We note that the superscripts like $I_{s_{n}-t_{n}}^{n}$ in (2.9) denote the transverse indices, while $t_{n}$ is the degree of the oscillator $\alpha_{n}$. In (2.9) and the subsequent expressions, $\alpha$ occurring in the argument of ket-vectors $|\phi(p, \alpha)\rangle$ denotes a set of the oscillators $\left\{\alpha_{n}^{I}, \alpha_{n}\right\}$, while $p$ occurring in the argument of ket-vectors $|\phi(p, \alpha)\rangle$ and $\delta$-functions denotes a set of the momenta $\left\{p^{I}, \beta \equiv p^{+}\right\}$. Also, we do not explicitly show the dependence of the ket-vectors $|\phi(p, \alpha)\rangle$ on the evolution parameter $x^{+}$. The ket-vector (2.9) is a degree $s_{n}$ homogeneous polynomial in the oscillators $\alpha_{n}^{I}, \alpha_{n}$ :

$$
\left(\alpha_{n}^{I} \bar{\alpha}_{n}^{I}+\alpha_{n} \bar{\alpha}_{n}-s_{n}\right)\left|\phi_{s_{1} \ldots s_{\nu}}(p, \alpha)\right\rangle=0, \quad n=1, \ldots, \nu .
$$

As noted above, physical D.o.F of a massive field in $d$-dimensional Minkowski space are described by irreps of the $s o(d-1)$ algebra. For the ket-vector (2.9) to be a carrier of $s o(d-1)$ algebra irreps, some constraints must be imposed on the ket-vector (2.9). But to avoid unnecessary complications, we do not impose any constraints on the tensor fields (2.9), which single out irreps of the $s o(d-1)$ algebra from these fields 5 . This implies that the ket-vector (2.9) actually describes a finite set of massive fields. To develop the light-cone gauge description of massive arbitrary spin fields on an equal footing we use a ket-vector defined by

$$
|\phi(p, \alpha)\rangle \equiv \sum_{s_{1}, \ldots, s_{\nu}=0}^{\infty}\left|\phi_{s_{1} \ldots s_{\nu}}(p, \alpha)\right\rangle
$$

Mixed-symmetry massless fields. The light-cone gauge description of a massless mixed-symmetry field can be realized by using a finite set of the creation and annihilation operators $\alpha_{n}^{I}$ and $\bar{\alpha}_{n}^{I}$ $(n=1,2, \ldots, \nu)$. In $d$-dimensional Minkowski space, the massless arbitrary spin field is labeled by spin labels $s_{1}, \ldots, s_{\nu}, \nu=\left[\frac{d-2}{2}\right]$ 6. Physical D.o.F of the massless field labeled by spin labels $s_{1}, \ldots, s_{\nu}$ can be collected into a ket-vector defined by

$$
\left|\phi_{s_{1} \ldots s_{\nu}}^{\mathrm{m}=0}(p, \alpha)\right\rangle \equiv \prod_{n=1}^{\nu} \alpha_{n}^{I_{1}^{n}} \ldots \alpha_{n}^{I_{s_{n}}^{n}} \phi_{s_{1} \ldots s_{\nu}}^{I_{1}^{1} \ldots I_{s_{1}}^{1} \ldots I_{1}^{\nu} \ldots I_{s_{\nu}}^{\nu}}(p)|0\rangle
$$

which is degree $s_{n}$ homogeneous polynomial in the oscillators $\alpha_{n}^{I}$ :

$$
\left(\alpha_{n}^{I} \bar{\alpha}_{n}^{I}-s_{n}\right)\left|\phi_{s_{1} \ldots s_{\nu}}^{\mathrm{m}=0}(p, \alpha)\right\rangle=0, \quad n=1, \ldots, \nu
$$

In $d$-dimensional Minkowski space, physical D.o.F of massless field are described by irreps of the $s o(d-2)$ algebra. For the ket-vector (2.12) to be a carrier of $s o(d-2)$ algebra irreps some additional constraints must be imposed on this ket-vector 7 . But, as in the case of a massive field, to avoid unnecessary complications we do not impose any constraints on the tensor fields (2.12);

\footnotetext{
${ }^{5}$ For even $d$, these constraints are: a) $\left(\alpha_{m}^{I} \bar{\alpha}_{n}^{I}+\alpha_{m} \bar{\alpha}_{n}-s_{n} \delta_{m n}\right)\left|\phi_{s_{1} \ldots s_{\nu}}\right\rangle=0, m \leq n$; b) $\left(\bar{\alpha}_{m}^{I} \bar{\alpha}_{n}^{I}+\right.$ $\left.\bar{\alpha}_{m} \bar{\alpha}_{n}\right)\left|\phi_{s_{1} \ldots s_{\nu}}\right\rangle=0$; c) $s_{1} \geq \ldots \geq s_{\nu-1} \geq s_{\nu} \geq 0, \nu=[(d-1) / 2]$. For odd $d$ and $s_{\nu}=0$, one can use the constraints a),b),c), while for $s_{\nu} \neq 0$ the label $s_{\nu}$ in a),c) should be replaced by $\left|s_{\nu}\right|$ and constraints a),b),c) should be supplemented by appropriate self-duality constraints. After imposing the constraints a),b),c) the labels $s_{1}, \ldots, s_{\nu}$ become Gelfand-Zetlin labels. In sections 2-7 we assume $s_{\nu} \geq 0$ and the constraints (2.10).

${ }^{6}$ In $4 d$ flat space, massless arbitrary spin field is labeled by one spin label $s=s_{1}$. Appearance of the spin labels $s_{1}, \ldots, s_{\nu}$ is related to the fact that physical D.o.F of massless field in $d$-dimensional flat space are described by $s o(d-2)$ algebra irreps labeled by $\left[\frac{d-2}{2}\right]$ Gelfand-Zetlin (or Dynkin) labels.

${ }^{7}$ For odd $d$, these constraints are: a) $\left(\alpha_{m}^{I} \bar{\alpha}_{n}^{I}-s_{n} \delta_{m n}\right)\left|\phi_{s_{1} \ldots s_{\nu}}\right\rangle=0, m \leq n$; b) $\bar{\alpha}_{m}^{I} \bar{\alpha}_{n}^{I}\left|\phi_{s_{1} \ldots s_{\nu}}\right\rangle=0$; c) $s_{1} \geq \ldots \geq s_{\nu-1} \geq s_{\nu} \geq 0, \nu=[(d-2) / 2]$. For even $d$ and $s_{\nu}=0$, one can use the constraints a),b),c), while for $s_{\nu} \neq 0$ the label $s_{\nu}$ in a),c) should be replaced by $\left|s_{\nu}\right|$ and constraints a),b),c) should be supplemented by appropriate self-duality constraints. In sections 2-7 we assume $s_{\nu} \geq 0$ and the constraints (2.13).
} 
therefore the ket-vector (2.12) describes a finite set of massless fields. By analogy with (2.11), the ket-vectors of massless fields (2.12) can be collected into a ket-vector $\left|\phi^{\mathrm{m}=0}(p, \alpha)\right\rangle$. We note that in (2.12) and the subsequent expressions, the letter $\alpha$ occurring in the argument of the ket-vectors of massless fields $\left|\phi^{\mathrm{m}=0}(p, \alpha)\right\rangle$ denotes a set of the oscillators $\alpha_{n}^{I}$.

Below, unless otherwise specified, we keep the integer $\nu$ to be arbitrary for flexibility.

Totally symmetric massive and massless fields. Totally symmetric fields are popular in various studies because these fields, being simpler than the mixed-symmetry fields, allow illustrating many characteristic features of higher spin fields in a relatively straightforward way. In order to obtain a description of massive and massless totally symmetric fields it is sufficient to introduce one sort of oscillators, i.e. we set $\nu=1$ in (2.9) and (2.12) respectively. This is to say that physical D.o.F. of massive and massless totally symmetric spin $s$ fields can be collected into the respective ket-vectors

$$
\begin{aligned}
& \left|\phi_{s}(p, \alpha)\right\rangle=\sum_{t=0}^{s} \alpha^{I_{1}} \ldots \alpha^{I_{s-t}} \alpha^{t} \phi^{I_{1} \ldots I_{s-t}}(p)|0\rangle, \\
& \left|\phi_{s}^{\mathrm{m}=0}(p, \alpha)\right\rangle=\alpha^{I_{1}} \ldots \alpha^{I_{s}} \phi^{I_{1} \ldots I_{s}}(p)|0\rangle .
\end{aligned}
$$

The ket-vector of massive field (2.14) is degree $s$ homogeneous polynomial in oscillators $\alpha^{I}, \alpha$, while the ket-vector of massless field (2.15) is degree $s$ homogeneous polynomial in oscillator $\alpha^{I}$ :

$$
\begin{aligned}
& \left(\alpha^{I} \bar{\alpha}^{I}+\alpha \bar{\alpha}-s\right)\left|\phi_{s}(p, \alpha)\right\rangle=0, \\
& \left(\alpha^{I} \bar{\alpha}^{I}-s\right) \mid \phi_{s}^{\mathrm{m}=0}(p, \alpha\rangle=0 .
\end{aligned}
$$

As was said in $d$-dimensional Minkowski space physical D.o.F of massive and massless fields are described by irreps of the $s o(d-1)$ and $s o(d-2)$ algebras respectively. In order for the fields (2.14) and (2.15) to realize irreps of the $s o(d-1)$ and $s o(d-2)$ algebras respectively we should impose the respective tracelessness constraints

$$
\left(\bar{\alpha}^{I} \bar{\alpha}^{I}+\bar{\alpha} \bar{\alpha}\right)\left|\phi_{s}(p, \alpha)\right\rangle=0, \quad \bar{\alpha}^{I} \bar{\alpha}^{I}\left|\phi_{s}^{\mathrm{m}=0}(p, \alpha)\right\rangle=0 .
$$

As in the case of mixed-symmetry fields in order to treat the totally symmetric arbitrary spin fields on an equal footing it is convenient to introduce ket-vectors for the respective towers of massive and massless fields

$$
\begin{aligned}
& |\phi(p, \alpha)\rangle \equiv \sum_{s=0}^{\infty}\left|\phi_{s}(p, \alpha)\right\rangle, \\
& \left|\phi^{\mathrm{m}=0}(p, \alpha)\right\rangle \equiv \sum_{s=0}^{\infty}\left|\phi_{s}^{\mathrm{m}=0}(p, \alpha)\right\rangle .
\end{aligned}
$$

We proceed with the discussion of a realization of the Poincare algebra on the space of massive and massless fields. A representation of the kinematical generators in terms of differential operators acting on the ket-vector $|\phi\rangle$ is given by 8

$$
\begin{array}{ll}
P^{I}=p^{I}, & P^{+}=\beta, \\
J^{+I}=\partial_{p^{I}} \beta, & J^{+-}=\partial_{\beta} \beta, \\
J^{I J}=p^{I} \partial_{p^{J}}-p^{J} \partial_{p^{I}}+M^{I J},
\end{array}
$$

\footnotetext{
${ }^{8}$ Throughout this paper, without loss of generality, we analyze generators of the Poincaré algebra and their commutators for $x^{+}=0$.
} 
where a spin operator $M^{I J}$ satisfies commutators of the $s o(d-2)$ algebra

$$
\left[M^{I J}, M^{K L}\right]=\delta^{J K} M^{I L}+3 \text { terms }
$$

and we use the notation

$$
\beta \equiv p^{+}, \quad \partial_{\beta} \equiv \partial / \partial \beta, \quad \partial_{p^{I}} \equiv \partial / \partial p^{I} .
$$

The representation of the dynamical generators in terms of differential operators acting on the ket-vector $|\phi\rangle$ is given by

$$
\begin{aligned}
& P^{-}=p^{-}, \quad p^{-} \equiv-\frac{p^{I} p^{I}+\mathrm{m}^{2}}{2 \beta}, \\
& J^{-I}=-\partial_{\beta} p^{I}+\partial_{p^{I}} P^{-}+\frac{1}{\beta}\left(M^{I J} p^{J}+\mathrm{m} M^{I}\right),
\end{aligned}
$$

where $\mathrm{m}$ is the mass parameter and $M^{I}$ is a spin operator transforming in the vector representation of the $s o(d-2)$ algebra. This operator satisfies the commutators

$$
\left[M^{I}, M^{J K}\right]=\delta^{I J} M^{K}-\delta^{I K} M^{J}, \quad\left[M^{I}, M^{J}\right]=-M^{I J} .
$$

The spin operators $M^{I J}$ and $M^{I}$ form commutators of the $s o(d-1)$ algebra (as it should be for the case of massive fields). The particular form of $M^{I J}$ and $M^{I}$ depends on the choice of the realization of spin D.o.F of physical fields. For example, a representation of the spin operators $M^{I J}$ and $M^{I}$ for the realization of the physical fields given in (2.11) takes the form

$$
M^{I J}=\sum_{n=1}^{\nu}\left(\alpha_{n}^{I} \bar{\alpha}_{n}^{J}-\alpha_{n}^{J} \bar{\alpha}_{n}^{I}\right), \quad M^{I}=\sum_{n=1}^{\nu}\left(\alpha_{n}^{I} \bar{\alpha}_{n}-\alpha_{n} \bar{\alpha}_{n}^{I}\right) .
$$

As seen from (2.27), in the limit as $\mathrm{m} \rightarrow 0$, the Poincaré algebra generators are independent of the spin operator $M^{I}$, i.e. the free light-cone gauge dynamics of massive fields have a smooth limit, given by the dynamics of massless fields.

The above expressions provide a realization of the Poincaré algebra in terms of differential operators acting on the physical field $|\phi\rangle$. We now write a field theoretical realization of this algebra in terms of the physical field $|\phi\rangle$. As mentioned above the kinematical generators $G^{k i n}$ are realized quadratically in $|\phi\rangle$, while the dynamical generators $G^{d y n}$ are realized non-linearly. At the quadratic level, both $G^{k i n}$ and $G^{d y n}$ admit the representation

$$
G_{[2]}=\int \beta d^{d-1} p\langle\phi(-p)|G| \phi(p)\rangle, \quad d^{d-1} p \equiv d \beta d^{d-2} p,
$$

where $G$ are the differential operators given in (2.21)-(2.23), (2.26), (2.27) and the notation $G_{[2]}$ is used for the field theoretical free generators. The field $|\phi\rangle$ satisfies the Poisson-Dirac commutator

$$
\begin{gathered}
{\left.\left[|\phi(p, \alpha)\rangle,\left|\phi\left(p^{\prime}, \alpha^{\prime}\right)\right\rangle\right]\right|_{\text {equal } x^{+}}=\frac{\delta^{d-1}\left(p+p^{\prime}\right)}{2 \beta}|\rangle|\rangle^{\prime}} \\
|\rangle|\rangle^{\prime} \equiv \exp \left(\sum_{n=1}^{\nu}\left(\alpha_{n}^{I} \alpha_{n}^{\prime I}+\alpha_{n} \alpha_{n}^{\prime}\right)\right)|0\rangle\left|0^{\prime}\right\rangle .
\end{gathered}
$$


With these definitions, we have the standard commutator

$$
\left[|\phi\rangle, G_{[2]}\right]=G|\phi\rangle .
$$

In the framework of the Lagrangian approach the light-cone gauge action takes the standard form

$$
S=\int d x^{+} d^{d-1} p\left\langle\phi(-p)\left|\mathrm{i} \beta \partial^{-}\right| \phi(p)\right\rangle+\int d x^{+} P^{-},
$$

where $P^{-}$is the Hamiltonian. This representation for the light-cone action is valid for the free and for the interacting theory. The free theory Hamiltonian can be obtained from relations (2.26), (2.30).

Incorporation of the internal symmetry into the theory under consideration resembles the ChanPaton method in string theory [47], and could be performed as in [48].

\section{General structure of $n$-point interaction vertices and light- cone dynamical principle}

We begin with discussing the general structure of the Poincaré algebra dynamical generators (2.5). In theories of interacting fields, the dynamical generators receive corrections involving higher powers of physical fields, and we have the following expansion for them:

$$
G^{d y n}=\sum_{n=2}^{\infty} G_{[n]}^{d y n}
$$

where $G_{[n]}^{d y n}$ stands for the $n$ - point contribution (i.e. the functional that has $n$ powers of physical fields) to the dynamical generator $G^{d y n}$. The generators $G^{d y n}$ of classical supersymmetric YangMills theories do not receive corrections of the order higher than four in fields [10, 11, 12], while the generators $G_{[n]}^{d y n}$ of (super)gravity theories are nontrivial for all $n \geq 2$ [49, 50, 51] 9 .

The 'free' generators $G_{[2]}^{d y n}($ 3.1), which are quadratic in the fields, were discussed in Section 2. Here we discuss the general structure of the 'interacting' dynamical generators $G_{[n]}^{d y n}, n \geq$ 3. Namely, we describe those properties of the dynamical generators $G_{[n]}^{d y n}, n \geq 3$, that can be obtained from commutators between $G^{k i n}$ and $G^{d y n}$. In other words, we find restrictions imposed by kinematical symmetries on the dynamical 'interacting' generators. We proceed in the following way.

(i) We first consider restrictions imposed by kinematical symmetries on the dynamical generator $P^{-}$. As seen from (2.1), the kinematical generators $P^{I}, P^{+}, J^{+I}$ have the following commutators with $P^{-}:\left[P^{-}, G_{[2]}^{k i n}\right]=G_{[2]}^{k i n}$. Since $G_{[2]}^{k i n}$ are quadratic in the fields, these commutators imply

$$
\left[P_{[n]}^{-}, G_{[2]}^{k i n}\right]=0, \quad n \geq 3 .
$$

\footnotetext{
${ }^{9}$ Generators of the closed string field theories, which involve the graviton field, terminate at cubic correction $G_{[3]}^{d y n}$ [8, 9]. It is natural to expect that generators of a general covariant theory should involve all powers of the graviton field $h_{\mu \nu}$. The fact that the closed string field theories do not involve vertices of the order higher than tree in $h_{\mu \nu}$ implies that the general covariance in these theories is realized in a nontrivial way. In string theory, the general covariance manifests itself upon integrating over massive string modes and going to the low energy expansion (see [52] for a discussion of this theme).
} 
Commutators (3.2) for $G_{[2]}^{k i n}=\left(P^{I}, P^{+}\right)$lead to the representation for $P_{[n]}^{-}$as

$$
P_{[n]}^{-}=\int d \Gamma_{n}\left\langle\Phi_{[n]} \mid p_{[n]}^{-}\right\rangle, \quad n \geq 3
$$

where we use the notation

$$
\begin{gathered}
\left\langle\Phi_{[n]}\left|\equiv \prod_{a=1}^{n}\left\langle\phi\left(p_{a}, \alpha_{a}\right)|, \quad| p_{[n]}^{-}\right\rangle \equiv p_{[n]}^{-} \prod_{a=1}^{n}\right| 0\right\rangle_{a}, \\
d \Gamma_{n} \equiv(2 \pi)^{d-1} \delta^{d-1}\left(\sum_{a=1}^{n} p_{a}\right) \prod_{a=1}^{n} \frac{d^{d-1} p_{a}}{(2 \pi)^{(d-1) / 2}} .
\end{gathered}
$$

Here and below, the indices $a, b=1, \ldots, n$ label $n$ interacting fields and the $\delta$ - functions in $d \Gamma_{n}$ (3.5) respect conservation laws for the transverse momenta $p_{a}^{I}$ and light-cone momenta $\beta_{a}$. Generic densities $p_{[n]}^{-}(3.4)$ depend on the momenta $p_{a}^{I}, \beta_{a}$, and variables related to the spin D.o.F, which we denote by $\alpha$ :

$$
p_{[n]}^{-}=p_{[n]}^{-}\left(p_{a}, \beta_{a} ; \alpha\right) .
$$

(ii) Commutators (3.2) for $G_{[2]}^{k i n}=J^{+I}$ tell us that the generic densities $p_{[n]}^{-}$in (3.4) depend on the momenta $p_{a}^{I}$ through the new momentum variables $\mathbb{P}_{a b}^{I}$ defined by

$$
\mathbb{P}_{a b}^{I} \equiv p_{a}^{I} \beta_{b}-p_{b}^{I} \beta_{a},
$$

i.e. the densities $p_{[n]}^{-}$turn out to be functions of $\mathbb{P}_{a b}^{I}$ in place of $p_{a}^{I} 10$ :

$$
p_{[n]}^{-}=p_{[n]}^{-}\left(\mathbb{P}_{a b}, \beta_{a} ; \alpha\right) .
$$

(iii) Commutators between $P^{-}$and the remaining kinematical generators $J^{I J}, J^{+-}$have the form $\left[P^{-}, J^{I J}\right]=0,\left[P^{-}, J^{+-}\right]=P^{-}$. Since $J^{I J}, J^{+-}$are quadratic in physical fields, these commutators lead to

$$
\left[P_{[n]}^{-}, J^{I J}\right]=0, \quad\left[P_{[n]}^{-}, J^{+-}\right]=P_{[n]}^{-}, \quad n \geq 3 .
$$

It is straightforward to check that commutators (3.9) lead to the respective equations for the generic densities $p_{[n]}^{-}=p_{[n]}^{-}\left(p_{a}, \beta_{a} ; \alpha\right)$ in (3.6):

$$
\begin{aligned}
& \sum_{a=1}^{n}\left(p_{a}^{I} \partial_{p_{a}^{J}}-p_{a}^{J} \partial_{p_{a}^{I}}+M^{(a) I J}\right)\left|p_{[n]}^{-}\right\rangle=0, \\
& \sum_{a=1}^{n} \beta_{a} \partial_{\beta_{a}}\left|p_{[n]}^{-}\right\rangle=0 .
\end{aligned}
$$

Using (3.7), we rewrite Eqs.(3.10), (3.11) in terms of $p_{[n]}^{-}=p_{[n]}^{-}\left(\mathbb{P}_{a b}, \beta_{a} ; \alpha\right)$ in (3.8) as

$$
\begin{aligned}
& \left(\sum_{\{a b\}} \mathbb{P}_{a b}^{I} \partial_{\mathbb{P}_{a b}^{J}}-\mathbb{P}_{a b}^{J} \partial_{\mathbb{P}_{a b}^{I}}+\sum_{a=1}^{n} M^{(a) I J}\right)\left|p_{[n]}^{-}\right\rangle=0, \\
& \left(\sum_{\{a b\}} \mathbb{P}_{a b}^{I} \partial_{\mathbb{P}_{a b}^{I}}+\sum_{a=1}^{n} \beta_{a} \partial_{\beta_{a}}\right)\left|p_{[n]}^{-}\right\rangle=0,
\end{aligned}
$$

\footnotetext{
${ }^{10}$ We note that due to momentum conservation laws not all $\mathbb{P}_{a b}^{I}$ are independent. It easy to check that the $n$-point vertex involves $n-2$ independent momenta $\mathbb{P}_{a b}^{I}$.
} 
where the notation $\{a b\}$ is used to label the $n-2$ independent momenta $\mathbb{P}_{a b}^{I}$.

(iv) To complete the description of the dynamical generators, we consider the dynamical generator $J^{-I}$. Using commutators of $J^{-I}$ with the kinematical generators, we obtain the representation for $J_{[n]}^{-I}, n \geq 3$ as

$$
J_{[n]}^{-I}=\int d \Gamma_{n}\left(\left\langle\Phi_{[n]} \mid j_{[n]}^{-I}\right\rangle+\frac{1}{n}\left(\sum_{a=1}^{n} \partial_{p_{a}^{I}}\left\langle\Phi_{[n]}\right|\right)\left|p_{[n]}^{-}\right\rangle\right),
$$

where we introduce new densities $j_{[n]}^{-I}$. From the commutators of $J^{-I}$ with the kinematical generators, we learn that the densities $j_{[n]}^{-I}$ depend on the momenta $p_{a}^{I}$ through the momenta $\mathbb{P}_{a b}^{I}$ in (3.7) and satisfy the equations

$$
\begin{aligned}
& \left(\sum_{\{a b\}} \mathbb{P}_{a b}^{I} \partial_{\mathbb{P}_{a b}^{J}}-\mathbb{P}_{a b}^{J} \partial_{\mathbb{P}_{a b}^{I}}+\sum_{a=1}^{n} M^{(a) I J}\right)\left|j_{[n]}^{-K}\right\rangle+\delta^{I K}\left|j_{[n]}^{-J}\right\rangle-\delta^{J K}\left|j_{[n]}^{-I}\right\rangle=0, \\
& \left(\sum_{\{a b\}} \mathbb{P}_{a b}^{I} \partial_{\mathbb{P}_{a b}^{I}}+\sum_{a=1}^{n} \beta_{a} \partial_{\beta_{a}}\right)\left|j_{[n]}^{-K}\right\rangle=0 .
\end{aligned}
$$

To summarize, the commutators between the kinematical and dynamical generators yield the expressions for the dynamical generators (3.3), (3.14), where the densities $p_{[n]}^{-}, j_{[n]}^{-I}$ depend on $\mathbb{P}_{a b}^{I}$, $\beta_{a}$, and spin variables $\alpha$ and satisfy Eqs.(3.12), (3.13), (3.15), (3.16).

To find the densities $p_{[n]}^{-}, j_{[n]}^{-I}$, we consider commutators between the respective dynamical generators; the general strategy of finding these densities consists basically of the following three steps, to be referred to as the light-cone dynamical principle:

a) Find restrictions imposed by commutators of the Poincaré algebra between the dynamical generators. Using these commutators shows that the densities $j_{[n]}^{-I}$ are expressible in terms of the densities $p_{[n]}^{-}$.

b) Require the densities $p_{[n]}^{-}, j_{[n]}^{-I}$ to be polynomials in the momenta $\mathbb{P}_{a b}^{I}$. We refer to this requirement as the light-cone locality condition.

c) Find those densities $p_{[n]}^{-}$that cannot be removed by field redefinitions.

In what follows, we apply the light-cone dynamical principle to study the density $p_{[3]}^{-}$, which we refer to as the cubic interaction vertex.

\section{Equations for cubic interaction vertices}

Although many examples of cubic interaction vertices are known in the literature, constructing cubic interaction vertices for concrete field theoretical models is still a challenging procedure. General methods essentially simplifying the procedure of obtaining cubic interaction vertices were discovered in [53, 54, 55]. In this section we develop the approach in Ref.[55] and demonstrate how our approach allows constructing cubic interaction vertices systematically and relatively straightforwardly.

As was explained above (see (3.8) $)$, the vertex $p_{[3]}^{-}$depends on the momenta $\mathbb{P}_{a b}^{I}$, where $a, b=$ $1,2,3$ label three interacting fields in the cubic interaction vertex. But the momenta $\mathbb{P}_{12}^{I}, \mathbb{P}_{23}^{I}, \mathbb{P}_{31}^{I}$ are not independent. This is, using the momentum conservation laws for $p_{a}^{I}$ and $\beta_{a}$,

$$
p_{1}^{I}+p_{2}^{I}+p_{3}^{I}=0, \quad \beta_{1}+\beta_{2}+\beta_{3}=0,
$$


it is easy to check that $\mathbb{P}_{12}^{I}, \mathbb{P}_{23}^{I}, \mathbb{P}_{31}^{I}$ can be expressed in terms of a new momentum $\mathbb{P}^{I}$ as

$$
\mathbb{P}_{12}^{I}=\mathbb{P}_{23}^{I}=\mathbb{P}_{31}^{I}=\mathbb{P}^{I}
$$

where the new momentum $\mathbb{P}^{I}$ is defined by

$$
\mathbb{P}^{I} \equiv \frac{1}{3} \sum_{a=1}^{3} \check{\beta}_{a} p_{a}^{I}, \quad \check{\beta}_{a} \equiv \beta_{a+1}-\beta_{a+2}, \quad \beta_{a} \equiv \beta_{a+3} .
$$

The use of $\mathbb{P}^{I}$ is advantageous since $\mathbb{P}^{I}$ is manifestly invariant under cyclic permutations of the external line indices $1,2,3$. Therefore the vertex $p_{[3]}^{-}$is eventually a function of $\mathbb{P}^{I}$ :

$$
p_{[3]}^{-}=p_{[3]}^{-}\left(\mathbb{P}, \beta_{a} ; \alpha\right) .
$$

Before discussing the restrictions imposed by the light-cone dynamical principle, we note that the kinematical symmetry equations (3.12), (3.13) take the following form in terms of vertex $p_{[3]}^{-}$(4.4):

$$
\begin{aligned}
& \mathbf{J}^{I J}\left|p_{[3]}^{-}\right\rangle=0, \\
& \left(\mathbb{P}^{I} \partial_{\mathbb{P}^{I}}+\sum_{a=1}^{3} \beta_{a} \partial_{\beta_{a}}\right)\left|p_{[3]}^{-}\right\rangle=0,
\end{aligned}
$$

where we use the notation

$$
\begin{aligned}
& \mathbf{J}^{I J} \equiv \mathbf{L}^{I J}(\mathbb{P})+\mathbf{M}^{I J}, \\
& \mathbf{L}^{I J}(\mathbb{P}) \equiv \mathbb{P}^{I} \partial_{\mathbb{P}^{J}}-\mathbb{P}^{J} \partial_{\mathbb{P}^{I}}, \quad \mathbf{M}^{I J} \equiv \sum_{a=1}^{3} M^{(a) I J} .
\end{aligned}
$$

We now proceed with discussing the restrictions imposed by the light-cone dynamical principle on vertex $p_{[3]}^{-}$(4.4). Following the procedure in the previous section, we first find the restrictions imposed by the Poincaré algebra commutators between the dynamical generators. All that is required is to consider the commutators

$$
\left[P^{-}, J^{-I}\right]=0, \quad\left[J^{-I}, J^{-J}\right]=0,
$$

which in the cubic approximation take the form

$$
\begin{aligned}
& {\left[P_{[2]}^{-}, J_{[3]}^{-I}\right]+\left[P_{[3]}^{-}, J_{[2]}^{-I}\right]=0,} \\
& {\left[J_{[2]}^{-I}, J_{[3]}^{-J}\right]+\left[J_{[3]}^{-I}, J_{[2]}^{-J}\right]=0 .}
\end{aligned}
$$

Equation (4.10) leads to the equation for the densities $\left|p_{[3]}^{-}\left(\mathbb{P}, \beta_{a} ; \alpha\right)\right\rangle$ and $\left|j_{[3]}^{-I}\left(\mathbb{P}, \beta_{a} ; \alpha\right)\right\rangle$,

$$
\mathbf{P}^{-}\left|j_{[3]}^{-I}\right\rangle=\mathbf{J}^{-I \dagger}\left|p_{[3]}^{-}\right\rangle,
$$

where we use the notation

$$
\begin{aligned}
& \mathbf{P}^{-} \equiv \sum_{a=1}^{3} p_{a}^{-}, \quad \mathbf{J}^{-I \dagger} \equiv \sum_{a=1}^{3} J_{a}^{-I \dagger}, \\
& p_{a}^{-} \equiv-\frac{p_{a}^{I} p_{a}^{I}+\mathrm{m}_{a}^{2}}{2 \beta_{a}}, \\
& J_{a}^{-I \dagger} \equiv p_{a}^{I} \partial_{\beta_{a}}-p_{a}^{-} \partial_{p_{a}^{I}}-\frac{1}{\beta_{a}}\left(M^{(a) I J} p_{a}^{J}+\mathrm{m}_{a} M^{(a) I}\right) .
\end{aligned}
$$


$\mathbf{P}^{-}$and the differential operator $\mathbf{J}^{-I \dagger}$ in (4.13) can be expressed in terms of the momentum $\mathbb{P}^{I}$ (see Appendix A):

$$
\begin{aligned}
& \mathbf{P}^{-} \equiv \frac{\mathbb{P}^{I} \mathbb{P}^{I}}{2 \hat{\beta}}-\sum_{a=1}^{3} \frac{\mathrm{m}_{a}^{2}}{2 \beta_{a}} \\
& \mathbf{J}^{-I \dagger}\left|p_{[3]}^{-}\right\rangle=-\frac{1}{3 \hat{\beta}} \mathcal{X}^{I}\left|p_{[3]}^{-}\right\rangle
\end{aligned}
$$

where we use the notation

$$
\begin{aligned}
& \mathcal{X}^{I} \equiv X^{I J} \mathbb{P}^{J}+X^{I}+X \partial_{\mathbb{P}^{I}} \\
& X^{I J} \equiv \sum_{a=1}^{3} \check{\beta}_{a}\left(\beta_{a} \partial_{\beta_{a}} \delta^{I J}-M^{(a) I J}\right) \\
& X^{I} \equiv \sum_{a=1}^{3} \frac{3 \hat{\beta} \mathrm{m}_{a}}{\beta_{a}} M^{(a) I} \\
& X \equiv-\sum_{a=1}^{3} \frac{\hat{\beta}_{\beta_{a}} \mathrm{~m}_{a}^{2}}{2 \beta_{a}} \\
& \hat{\beta} \equiv \beta_{1} \beta_{2} \beta_{3} .
\end{aligned}
$$

Taking (4.17) into account we can rewrite Eq.(4.12) as

$$
\left|j_{[3]}^{-I}\right\rangle=-\frac{1}{3 \hat{\beta} \mathbf{P}^{-}} \mathcal{X}^{I}\left|p_{[3]}^{-}\right\rangle,
$$

which tells us that the density $j_{[3]}^{-I}$ is not an independent quantity but is expressible in terms of vertex $p_{[3]}^{-}$(4.4). By substituting $j_{[3]}^{-I}$ (4.23) into Eq.(4.11), we can verify that Eq.(4.11) is fulfilled. Thus, we exhaust all commutators of the Poincaré algebra in the cubic approximation. Equations (4.5), (4.6) supplemented by relation (4.23) provide the complete list of restrictions imposed by commutators of the Poincaré algebra on the densities $p_{[3]}^{-}, j_{[3]}^{-I}$. We see that the restrictions imposed by commutators of the Poincare algebra by themselves are not sufficient to fix the vertex $p_{[3]}^{-}$uniquely. To choose the physically relevant densities $p_{[3]}^{-}, j_{[3]}^{-I}$, i.e. to fix them uniquely, we impose the light-cone locality condition: we require the densities $p_{[3]}^{-}, j_{[3]}^{-I}$ to be polynomials in $\mathbb{P}^{I}$. As regards the vertex $p_{[3]}^{-}$, we require this vertex to be local (i.e. polynomial in $\mathbb{P}^{I}$ ) from the very beginning. However it is clear from relation (4.23) that a local $p_{[3]}^{-}$does not lead automatically to a local density $j_{[3]}^{-I}$. From (4.23), we see that the light-cone locality condition for $j_{[3]}^{-I}$ amounts to the equation

$$
\mathcal{X}^{I}\left|p_{[3]}^{-}\right\rangle=\mathbf{P}^{-}\left|V^{I}\right\rangle,
$$

where a vertex $\left|V^{I}\right\rangle$ is restricted to be polynomial in $\mathbb{P}^{I}$. In fact, imposing the light-cone locality condition amounts to requiring that the generators of the Poincaré algebra be local functionals of the physical fields with respect to transverse directions.

The last requirement we impose on the cubic interaction vertex is related to field redefinitions. We note that by using local (i.e. polynomial in the transverse momenta) field redefinitions, we 
can remove the terms in the vertex $p_{[3]}^{-}$that are proportional to $\mathbf{P}^{-}$(see Appendix $\mathrm{B}$ ). Since we are interested in the vertex that cannot be removed by field redefinitions, we impose the equation

$$
\left|p_{[3]}^{-}\right\rangle \neq \mathbf{P}^{-}|V\rangle
$$

where a vertex $|V\rangle$ is restricted to be polynomial in $\mathbb{P}^{I}$. We note that Eqs.(4.24), (4.25) amount to the light-cone dynamical principle. If we restrict ourselves to low spin $s=1,2$ field theories, i.e. Yang-Mills and Einstein theories, it can then be verified that the light-cone dynamical principle and Eqs.(4.5), (4.6) allow fixing the cubic interaction vertices unambiguously (up to several coupling constants). It then seems reasonable to use the light-cone dynamical principle and Eqs.(4.5), (4.6) for studying the cubic interaction vertices of higher spin fields.

To summarize the discussion in this section, we collect equations imposed by the kinematical symmetries and the light-cone dynamical principle on vertex $p_{[3]}^{-}(4.4)$ :

$$
\begin{array}{ll}
\mathbf{J}^{I J}\left|p_{[3]}^{-}\right\rangle=0, & \text { so }(d-2) \text { invariance } \\
\left(\mathbb{P}^{I} \partial_{\mathbb{P}}+\sum_{a=1}^{3} \beta_{a} \partial_{\beta_{a}}\right)\left|p_{[3]}^{-}\right\rangle=0, & \beta-\text { homogeneity } \\
\mathcal{X}^{I}\left|p_{[3]}^{-}\right\rangle=\mathbf{P}^{-}\left|V^{I}\right\rangle, & \text { light-cone locality condition } \\
\left|p_{[3]}^{-}\right\rangle \neq \mathbf{P}^{-}|V\rangle, &
\end{array}
$$

where the vertices $|V\rangle$ and $\left|V^{I}\right\rangle$ are restricted to be polynomials in $\mathbb{P}^{I}$. Solving light-cone locality condition (4.28) leads to the representation for the density $\left|j_{[3]}^{-I}\right\rangle(4.23)$ as

$$
\left|j_{[3]}^{-I}\right\rangle=-\frac{1}{3 \hat{\beta}}\left|V^{I}\right\rangle \text {. }
$$

Equations (4.26)-(4.29) constitute a complete system of equations on vertex $p_{[3]}^{-}$(4.4). Equation (4.26) reflects the invariance of the vertex $\left|p_{[3]}^{-}\right\rangle$under $s o(d-2)$ rotations, and Eq.(4.27) tells us that $\left|p_{[3]}^{-}\right\rangle$is a zero-degree homogeneity function in $\mathbb{P}^{I}$ and $\beta_{a}$. Equations (4.28), (4.29) and the representation for the density $\left|j_{[3]}^{-I}\right\rangle$ (4.30) are obtainable from the light-cone dynamical principle.

\subsection{Equations for cubic interaction vertices in the harmonic scheme for an arbitrary realization of the spin degrees of freedom}

Kinematical symmetry equations (4.26), (4.27) present no difficulties. For example, the solution of Eq.(4.26) can be written simply as $p_{[3]}^{-}=p_{[3]}^{-}(\mathcal{I})$, where $\mathcal{I}$ is the complete set of the $s o(d-2)$ algebra invariants, which can be constructed using $\mathbb{P}^{I}$ and the variables describing spin degrees of freedom. A real difficulty is then to choose such a $p_{[3]}^{-}(\mathcal{I})$ that satisfies the light-cone locality condition (4.28) and Eq.(4.29). Since Eqs.(4.28), (4.29) are inconvenient to use it is preferable to recast these equations into some explicit differential equations. In turns out that this becomes possible by using the special, so called harmonic scheme. Our purpose now is therefore to formulate equations for the cubic interaction vertex in the harmonic scheme.

We begin with defining the harmonic scheme. By definition, the vertex $\left|p_{[3]}^{-}\right\rangle$is a polynomial in the momentum $\mathbb{P}^{I}$. It is well known that an arbitrary polynomial in $\mathbb{P}^{I}$ can be made a harmonic polynomial in $\mathbb{P}^{I}$ by adding a suitable polynomial proportional to $\mathbb{P}^{I} \mathbb{P}^{I}$. We also recall that a polynomial proportional to $\mathbb{P}^{I} \mathbb{P}^{I}$ can be generated using field redefinitions. In other words, via 
field redefinitions, the vertex $\left|p_{[3]}^{-}\right\rangle$can be made a harmonic polynomial in $\mathbb{P}^{I}$ (see Appendix B). The scheme in which the vertex $\left|p_{[3]}^{-}\right\rangle$satisfies the harmonic equation

$$
\partial_{\mathbb{P}^{I}} \partial_{\mathbb{P}^{I}}\left|p_{[3]}^{-}\right\rangle=0
$$

is referred to as the harmonic scheme.

We proceed with the discussion of Eqs.(4.28), (4.29) in the harmonic scheme. In the harmonic scheme, Eq.(4.29) is satisfied automatically because a harmonic polynomial in $\mathbb{P}^{I}$ cannot be represented as $\mathbf{P}^{-}|V\rangle$, where $|V\rangle$ is a polynomial in $\mathbb{P}^{I}$. It then remains to analyze the light-cone locality condition (4.28) in the harmonic scheme. It turns out that this condition leads to the differential equations for vertex $p_{[3]}^{-}$(4.4) (see Appendix C):

$$
\left(X^{I J} \mathcal{P}^{J}+X^{I}+\left(X \delta^{I J}+\frac{\hat{\beta}}{2 \widehat{k}^{\prime}+N} \sum_{a=1}^{3} \frac{\mathrm{m}_{a}^{2}}{\beta_{a}} X^{I J}\right) \partial_{\mathbb{P}^{J}}\right)\left|p_{[3]}^{-}\right\rangle=0,
$$

where $X^{I J}, X^{I}, X$ are defined in (4.19)-(4.21) and we use the notation

$$
\mathcal{P}^{I} \equiv \mathbb{P}^{I}-\mathbb{P}^{J} \mathbb{P}^{J} \frac{1}{2 \widehat{k}+N} \partial_{\mathbb{P}^{I}}, \quad \widehat{k} \equiv \mathbb{P}^{I} \partial_{\mathbb{P}^{I}}, \quad N \equiv d-2
$$

In what follows, we refer to Eqs.(4.32) as locality equations. A remarkable property of the harmonic scheme is that it allows writing closed expression for the density $\left|j_{[3]}^{-I}\right\rangle$ in terms of vertex $\left|p_{[3]}^{-}\right\rangle(4.4)$ without specifying the spin operators $M^{I J}, M^{I}$ :

$$
\left|j_{[3]}^{-I}\right\rangle=-\frac{2}{3(2 \widehat{k}+N)} X^{I J} \partial_{\mathbb{P}^{J}}\left|p_{[3]}^{-}\right\rangle .
$$

To summarize, the complete set of equations to be solved in the harmonic scheme is given by (4.26), (4.27), (4.31), (4.32). These equations are used in Section 8 to develop the so called $s o(d-$ 4) light-cone approach.

\subsection{Equations for parity invariant cubic interaction vertices in the minimal scheme and for the oscillator realization of spin degrees of freedom}

In this section, we develop an alternative approach to the analysis of the equations for the cubic interaction vertex (4.26)-(4.29) based on a scheme we refer to as the minimal scheme. This scheme, to be defined below, turns out to be convenient for the classification of cubic interaction vertices.

To proceed, we use the oscillator realization of physical fields in terms of the ket-vectors in (2.9), (2.11). In this case, the spin arguments of vertex $p_{[3]}^{-}$(4.4) denoted by $\alpha$ become oscillators $\alpha_{n}^{(a) I}, \alpha_{n}^{(a)}, a=1,2,3$, and vertex $p_{[3]}^{-}(4.4)$ takes the form 11 :

$$
\left|p_{[3]}^{-}\right\rangle=p_{[3]}^{-}\left(\mathbb{P}^{I}, \beta_{a} ; \alpha_{n}^{(a) I}, \alpha_{n}^{(a)}\right)|0\rangle_{1}|0\rangle_{2}|0\rangle_{3} .
$$

We now analyze Eqs.4.26)-(4.29) in turn.

i) We first analyze the restrictions imposed by the $s o(d-2)$ invariance equations (4.26). These equations tell us that vertex $p_{[3]}^{-}(4.35)$ depends on the $s o(d-2)$ algebra invariants that can be

\footnotetext{
${ }^{11}$ Throughout this paper, unless otherwise specified, the subscripts $m, n, q$ takes the values $1, \ldots, \nu$. The short notation like $p_{[3]}^{-}\left(x^{(a)}\right)$ is used to indicate the dependence of $p_{[3]}^{-}$on $x^{(1)}, x^{(2)}, x^{(3)}$.
} 
constructed using $\mathbb{P}^{I}$ and the oscillators $\alpha_{n}^{(a) I}$. It is clear that the following $s o(d-2)$ invariants can be constructed:

$$
\mathbb{P}^{I} \mathbb{P}^{I}, \quad \alpha_{n}^{(a) I} \mathbb{P}^{I}, \quad \alpha_{n}^{(a) I} \alpha_{m}^{(b) I} .
$$

We note that there are additional invariants constructed using the antisymmetric Levi-Civita symbol $\epsilon^{I_{1} \ldots I_{d-2}}$. The vertices not involving the antisymmetric Levi-Civita symbol are said to be parity invariant vertices 12 , and vertices involving one antisymmetric Levi-Civita symbol are said to be parity violating vertices. We focus on the parity invariant vertices (below we shall discuss the cases where the parity invariant vertices provide a complete list of vertices). Thus, we restrict our attention to the vertex

$$
p_{[3]}^{-}=p_{[3]}^{-}\left(\mathbb{P}^{I} \mathbb{P}^{I}, \beta_{a} ; \alpha_{n}^{(a) I} \mathbb{P}^{I}, \alpha_{n}^{(a)} ; \alpha_{m n}^{(a a+1)}, \alpha_{m n}^{(a a)}\right),
$$

where

$$
\alpha_{m n}^{(a b)} \equiv \alpha_{m}^{(a) I} \alpha_{n}^{(b) I}
$$

ii) The second step is to analyze the restrictions imposed by Eq.4.29). Using field redefinitions, we can remove the terms in (4.37) that are proportional to $\mathbb{P}^{I} \mathbb{P}^{I}$. Thus, we can drop down the dependence on $\mathbb{P}^{I} \mathbb{P}^{I}$ in $p_{[3]}^{-}$(4.37). The scheme in which the vertex $p_{[3]}^{-}$is independent of $\mathbb{P}^{I} \mathbb{P}^{I}$ is said to be the minimal scheme. Obviously, in the minimal scheme, vertex $p_{[3]}^{-}$(4.37), being polynomial in $\mathbb{P}^{I}$, satisfies Eq.(4.29) automatically.

Before analyzing the light-cone locality condition, in place of the variables used in (4.37),

$$
\beta_{a}, \quad \alpha_{n}^{(a) I} \mathbb{P}^{I}, \quad \alpha_{n}^{(a)}, \quad \alpha_{m n}^{(a a+1)}, \quad \alpha_{m n}^{(a a)},
$$

we introduce the new variables

$$
\beta_{a}, \quad B_{n}^{(a)}, \quad \alpha_{n}^{(a)}, \quad \alpha_{m n}^{(a a+1)}, \quad Q_{m n}^{(a a)},
$$

where the new 'improved' $s o(d-2)$ invariants $B_{m}^{(a)}, Q_{m n}^{(a a)}$ are defined by

$$
\begin{aligned}
B_{n}^{(a)} & \equiv \frac{\alpha_{n}^{(a) I} \mathbb{P}^{I}}{\beta_{a}}+\frac{\check{\beta}_{a}}{2 \beta_{a}} \mathrm{~m}_{a} \alpha_{n}^{(a)}, \\
Q_{m n}^{(a a)} & \equiv \alpha_{m n}^{(a a)}+\alpha_{m}^{(a)} \alpha_{n}^{(a)} .
\end{aligned}
$$

The use of the variables $Q_{m n}^{(a a)}(4.40)$ instead of $\alpha_{m n}^{(a a)}$ (4.39) is preferable because the variables $Q_{m n}^{(a a)}$ commute with the spin operators of the $s o(d-1)$ algebra

$$
M^{(a) I J}=\sum_{n=1}^{\nu}\left(\alpha_{n}^{(a) I} \bar{\alpha}_{n}^{(a) J}-\alpha_{n}^{(a) J} \bar{\alpha}_{n}^{(a) I}\right), \quad M^{(a) I}=\sum_{n=1}^{\nu}\left(\alpha_{n}^{(a) I} \bar{\alpha}_{n}^{(a)}-\alpha_{n}^{(a)} \bar{\alpha}_{n}^{(a) I}\right),
$$

i.e. $Q_{m n}^{(a a)}$ are invariants of the $s o(d-1)$ algebra. The advantages of the variables $B_{n}^{(a)}(4.40)$ over the variables $\alpha_{n}^{(a) I} \mathbb{P}^{I}(4.39)$ are to be explained shortly in the course of the analysis of the light-cone locality condition. Thus, we use the 'improved' representation for the vertex

$$
p_{[3]}^{-}=p_{[3]}^{-}\left(\beta_{a}, B_{n}^{(a)}, \alpha_{n}^{(a)} ; \alpha_{m n}^{(a a+1)}, Q_{m n}^{(a a)}\right) .
$$

\footnotetext{
12 The methods of manifest covariantization of light-cone vertices [15]-[19] are most suitable for studying the parity invariant vertices. Thus, we expect that all our parity invariant vertices could be covariantized in a relatively straightforward way.
} 
iii) We next analyze the restrictions imposed by the light-cone locality condition (4.28). For this, we derive the following formula for action of the operator $\mathcal{X}^{I}(4.18)$ on vertex $p_{[3]}^{-}(4.44)$ :

$$
\frac{1}{3 \hat{\beta}} \mathcal{X}^{I}\left|p_{[3]}^{-}\right\rangle=-\mathbf{P}^{-} \sum_{\substack{a=1,2,3 \\ n=1, \ldots, \nu}} \frac{2 \check{\beta}_{a}}{3 \beta_{a}} \alpha_{n}^{(a) I} \partial_{B_{n}^{(a)}}\left|p_{[3]}^{-}\right\rangle+\sum_{\substack{a=1,2,3 \\ n=1, \ldots, \nu}} \frac{1}{\beta_{a}} \alpha_{n}^{(a) I} G_{a n}+\mathbb{P}^{I} E,
$$

where we use the notation

$$
\begin{aligned}
G_{a n} & \equiv\left\{-\frac{1}{2}\left(\mathrm{~m}_{a+1}^{2}-\mathrm{m}_{a+2}^{2}\right) \partial_{B_{n}^{(a)}}+\mathrm{m}_{a} \partial_{\alpha_{n}^{(a)}}\right. \\
& \left.+\sum_{m=1}^{\nu}\left(B_{m}^{(a+1)}+\frac{1}{2} \mathrm{~m}_{a+1} \alpha_{m}^{(a+1)}\right) \partial_{\alpha_{n m}^{(a+1)}}-\left(B_{m}^{(a+2)}-\frac{1}{2} \mathrm{~m}_{a+2} \alpha_{m}^{(a+2)}\right) \partial_{\alpha_{m n}^{(a+2 a)}}\right\}\left|p_{[3]}^{-}\right\rangle, \\
E & \equiv \frac{1}{3 \hat{\beta}} \sum_{a=1}^{3} \check{\beta}_{a} \beta_{a} \partial_{\beta_{a}}\left|p_{[3]}^{-}\right\rangle .
\end{aligned}
$$

It follows from relation (4.45) that the light-cone locality condition (4.28) amounts to the equations

$$
\begin{aligned}
& G_{a n}=0, \quad a=1,2,3 ; \quad n=1, \ldots, \nu ; \\
& E=0 .
\end{aligned}
$$

We note that in deriving relation (4.45) we use the fact that the operator $\mathcal{X}^{I}$ does not act on the variables $Q_{m n}^{(a a)}$ because these variables commute with the spin operators (4.43). The use of the variables $B_{n}^{(a)}$ is advantageous because $B_{n}^{(a)}$ satisfy the equations

$$
\begin{aligned}
& \left(\mathbb{P}^{I} \partial_{\mathbb{P} I}+\sum_{a=1}^{3} \beta_{a} \partial_{\beta_{a}}\right) B_{n}^{(b)}=0, \\
& \sum_{a=1}^{3}\left\{\check{\beta}_{a}\left(\mathbb{P}^{I} \beta_{a} \partial_{\beta_{a}}+\sum_{n=1}^{\nu} \mathbb{P}^{J} \alpha_{n}^{(a) J} \bar{\alpha}_{n}^{(a) I}\right)-\frac{3 \hat{\beta} \mathrm{m}_{a}}{\beta_{a}} \sum_{n=1}^{\nu} \alpha_{n}^{(a)} \bar{\alpha}_{n}^{(a) I}\right\} B_{m}^{(b)}|0\rangle=0 .
\end{aligned}
$$

Equations (4.50) and (4.51) are very helpful for solving the $\beta$-homogeneity equation (4.27) and deriving formula (4.45) respectively.

iv) We finally analyze the restrictions imposed by the $\beta$-homogeneity equation (4.27) and Eq.(4.49). In terms of vertex $p_{[3]}^{-}$(4.44), Eqs.(4.27), (4.49) become

$$
\sum_{a=1}^{3} \beta_{a} \partial_{\beta_{a}} p_{[3]}^{-}=0, \quad \sum_{a=1}^{3} \check{\beta}_{a} \beta_{a} \partial_{\beta_{a}} p_{[3]}^{-}=0 .
$$

Equations (4.52) imply that vertex $p_{[3]}^{-}$(4.44) is independent of $\beta_{a}, a=1,2,3$ :

$$
p_{[3]}^{-}=p_{[3]}^{-}\left(B_{n}^{(a)}, \alpha_{n}^{(a)}, \alpha_{m n}^{(a a+1)}, Q_{m n}^{(a a)}\right),
$$

while Eqs.(4.23), (4.45), (4.48), (4.49) lead to the following expression for $\left|j_{[3]}^{-I}\right\rangle$ :

$$
\left|j_{[3]}^{-I}\right\rangle=\sum_{\substack{a=1,2,3 \\ n=1, \ldots, \nu}} \frac{2 \check{\beta}_{a}}{3 \beta_{a}} \alpha_{n}^{(a) I} \partial_{B_{n}^{(a)}}\left|p_{[3]}^{-}\right\rangle .
$$


Summary of analysis in Eqs.44.26)-(4.29) in the minimal scheme is given in formula (4.53), with the equations still to be solved given in (4.48). Up to this point our treatment has been applied to vertices for massive as well as massless fields. From now on, we separately consider vertices for the massless fields, vertices involving both massless and massive fields, and vertices for the massive fields. The vertices in Sections 5, 7 constitute the complete lists of parity invariant cubic interaction vertices for massless and massive fields in $d \geq 4$ dimensions.

\section{Parity invariant cubic interaction vertices for massless fields}

We begin with discussing the parity invariant cubic interaction vertex for the massless mixedsymmetry fields. We consider vertex (4.53) for three massless fields:

$$
\mathrm{m}_{1}=\mathrm{m}_{2}=\mathrm{m}_{3}=0 .
$$

Equations for the vertex involving three massless fields are obtainable from Eqs.(4.48) by letting $\mathrm{m}_{a} \rightarrow 0, a=1,2,3$, in Eqs.(4.48). The general solution for vertex (4.53) then takes the form (see Appendix D)

$$
p_{[3]}^{-}=p_{[3]}^{-}\left(B_{n}^{(a)} ; \alpha_{m n}^{(a a)} ; Z_{m n q}\right),
$$

where we use the notation

$$
B_{n}^{(a)} \equiv \frac{\alpha_{n}^{(a) I} \mathbb{P}^{I}}{\beta_{a}}, \quad Z_{m n q} \equiv B_{m}^{(1)} \alpha_{n q}^{(23)}+B_{n}^{(2)} \alpha_{q m}^{(31)}+B_{q}^{(3)} \alpha_{m n}^{(12)},
$$

and $\alpha_{m n}^{(a b)}$ is defined in (4.38). This solution provides the complete list of parity invariant cubic interaction vertices for both totally symmetric and mixed-symmetry fields.

We now comment on the solution obtained. Vertex $p_{[3]}^{-}(5.2)$ depends on $B_{n}^{(a)}, \alpha_{m n}^{(a a)}$ and $Z_{m n q}$, which are the respective degree 1, 2, and 3 homogeneous polynomials in oscillators. Henceforth, degree 1,2, and 3 homogeneous polynomials in oscillators are referred to as linear, quadratic, and cubic forms respectively. We emphasize, however, that the contribution of the $\alpha_{m n}^{(a a)}$-terms to the Hamiltonian $P_{[3]}^{-}$vanishes when the ket-vector (2.12) is subjected to the tracelessness constraint. This is, the physical massless fields, being irreps of the $s o(d-2)$ algebra, satisfy the tracelessness constraints

$$
\bar{\alpha}_{m}^{(a) I} \bar{\alpha}_{n}^{(a) I}\left|\phi_{a}^{\mathrm{m}_{a}=0}\right\rangle=0, \quad a=1,2,3 .
$$

It is then clear that the $\alpha_{m n}^{(a a)}$-terms in (5.2) do not contribute to the Hamiltonian $P_{[3]}^{-}(3.3)^{13}$. Thus, in case of massless fields belonging to irreps of the $s o(d-2)$ algebra, vertex (5.2) is governed by the linear forms $B_{n}^{(a)}$ and by the cubic forms $Z_{m n q}$. To understand the remaining important properties of solution (5.2) we consider cubic vertices for massless totally symmetric fields.

\subsection{Cubic interaction vertices for massless totally symmetric fields}

In this section we restrict attention to the parity invariant cubic interaction vertices for massless totally symmetric fields. To consider the totally symmetric fields it suffices to use one sort of

\footnotetext{
${ }^{13}$ We keep $\alpha_{m n}^{(a a)}$-terms in the general solution (5.2) because in certain applications it is convenient to deal with ketvectors $\left|\phi^{\mathrm{m}=0}\right\rangle$, which are not subjected to the tracelessness constraint (5.4). It is clear that such ket-vectors describe a collection of massless fields.
} 
oscillators, i.e. to set $\nu=1$ in (5.2), (5.3). To simplify formulas we drop oscillator's subscript $n=1$ and use the simplified notation $\alpha^{I} \equiv \alpha_{1}^{I}$. The cubic interaction vertex can then be obtained from the general solution (5.2) by using the identifications

$$
\alpha^{(a) I} \equiv \alpha_{1}^{(a) I}, \quad a=1,2,3,
$$

in (5.2) and ignoring contribution of oscillators carrying a subscript $n>1$. Adopting the simplified notation (5.5) for linear forms $B^{(a)} \equiv B_{1}^{(a)}$ (5.3), quadratic forms $\alpha^{(a b)} \equiv \alpha_{11}^{(a b)}(4.38)$, and cubic form $Z \equiv Z_{111}($ (5.3), we obtain

$$
B^{(a)} \equiv \frac{\alpha^{(a) I} \mathbb{P}^{I}}{\beta_{a}}, \quad \alpha^{(a b)} \equiv \alpha^{(a) I} \alpha^{(b) I}, \quad Z \equiv \sum_{a=1}^{3} B^{(a)} \alpha^{(a+1 a+2)}
$$

and vertex (5.2) becomes

$$
p_{[3]}^{-}=p_{[3]}^{-}\left(B^{(a)} ; \alpha^{(a a)} ; Z\right) .
$$

Vertex (5.7) describes interaction of towers of massless fields (2.20). We now obtain vertex for massless totally symmetric $\operatorname{spin} s^{(1)}, s^{(2)}, s^{(3)}$ fields. The massless totally symmetric spin $s^{(a)}$ fields are described by the respective ket-vectors $\left|\phi_{s^{(a)}}^{\mathrm{m}_{a}=0}\right\rangle$. The ket-vectors of massless fields $\left|\phi_{s_{a}(a)}^{\mathrm{m}_{a}=0}\right\rangle$, $a=1,2,3$, are obtainable from (2.15) by replacement $s \rightarrow s^{(a)}, \alpha^{I} \rightarrow \alpha^{(a) I}$ in (2.15). Taking into account that the ket-vectors $\left|\phi_{s^{(a)}}^{\mathrm{m}_{a}=0}\right\rangle$ are the respective degree $s^{(a)}$ homogeneous polynomials in the oscillators $\alpha^{(a) I}$ (see (2.17) ) it is easy to see that the vertex we are interested in must satisfy the equations

$$
\left(\alpha^{(a) I} \bar{\alpha}^{(a) I}-s^{(a)}\right)\left|p_{[3]}^{-}\right\rangle=0, \quad a=1,2,3,
$$

which tell us that the vertex $p_{[3]}^{-}$should be degree $s^{(a)}$ homogeneous polynomial in the oscillators $\alpha^{(a) I}$. Taking into account that the forms $B^{(a)}$ and $Z(5.6)$ are the respective degree 1 and 3 homogeneous polynomials in oscillators, we find the general solution of Eq.(5.8)

$$
p_{[3]}^{-}\left(s^{(1)}, s^{(2)}, s^{(3)} ; k\right)=Z^{\frac{1}{2}(\mathbf{s}-k)} \prod_{a=1}^{3}\left(B^{(a)}\right)^{s^{(a)}+\frac{1}{2}(k-\mathbf{s})},
$$

where we use the notation 14

$$
\mathbf{s} \equiv \sum_{a=1}^{3} s^{(a)}
$$

and integer $k$ is a freedom in our solution. The integer $k$ labels all possible cubic vertices that can be built for massless spin $s^{(1)}, s^{(2)}, s^{(3)}$ fields and has a clear physical interpretation. Taking into account that the forms $B^{(a)}$ and $Z$ (5.6) are degree 1 homogeneous polynomials in the momentum $\mathbb{P}^{I} 15$ it is easy to see that vertex (5.9) is a degree $k$ homogeneous polynomial in $\mathbb{P}^{I}$. To summarize, the vertex $p_{[3]}^{-}\left(s^{(1)}, s^{(2)}, s^{(3)} ; k\right)$ describes interaction of three massless spin $s^{(1)}, s^{(2)}, s^{(3)}$ fields having

\footnotetext{
${ }^{14}$ We ignore the contribution of $\alpha^{(a a)}$-terms (5.7) to vertex (5.9). Because of the tracelessness constraint (see the second relation in (2.18) ) the contribution of these terms to the Hamiltonian $P_{[3]}^{-}(3.3)$ vanishes.

${ }^{15}$ It is this property of the forms $B^{(a)}$ and $Z$ that allows us to introduce the vertex that is the homogeneous polynomial in $\mathbb{P}^{I}$. A completely different type of a situation occurs in the case of massive fields, whose cubic interaction vertices depend on forms that are non-homogeneous polynomials in the $\mathbb{P}^{I}$.
} 
$k$ powers of the momentum $\mathbb{P}^{I}$. In the Lorentz covariant approach, the integer $k$ is equal to the number of the derivatives with respect to space-time coordinates.

We now discuss the restrictions to be imposed on the spin values $s^{(1)}, s^{(2)}, s^{(3)}$ and the integer $k$. The powers of the forms $B^{(a)}$ and $Z$ in (5.9) must be non-negative integers. For this to be the case, it is necessary to impose the following restrictions on the allowed spin values $s^{(1)}, s^{(2)}, s^{(3)}$ and the number of powers of the momentum $\mathbb{P}^{I}$ (the number of the derivatives):

$$
\begin{array}{cc}
k \leq \mathbf{s}, & \mathbf{s}-k \leq 2 s^{(a)}, \quad a=1,2,3, \\
\mathbf{s}-k & \text { even integer. }
\end{array}
$$

Restrictions (5.11) can be rewritten equivalently as

$$
\mathbf{s}-2 s_{\min } \leq k \leq \mathbf{s}, \quad s_{\min } \equiv \min _{a=1,2,3} s^{(a)} .
$$

A few remarks are in order.

i) The restriction $k \leq \mathrm{s}$ in (5.13) expresses the fact that in the minimal scheme, which does not admit $\mathbb{P}^{I} \mathbb{P}^{I}$-terms, the total number of the transverse indices of fields that enter the cubic Hamiltonian $P_{[3]}^{-}$cannot be less than the number of powers of the momentum $\mathbb{P}^{I}$ in the vertex.

ii) If $k=2$, then the restriction $\mathbf{s}-2 s_{\min } \leq 2$ in $(5.13)$ is precisely the restriction that leaves no place for the gravitational interaction of massless higher spin fields $(s>2)$. Indeed, the gravitational interaction of a massless spin $s$ field could be described by the vertex $p_{[3]}^{-}\left(s^{(1)}, s^{(2)}, s^{(3)} ; k\right)$ with $s^{(1)}=s^{(2)}=s>2, s^{(3)}=2, k=2$. For these values $s^{(a)}$ we obtain $s_{\text {min }}=2, \mathbf{s}=2 s+2$ and therefore restrictions (5.13) take the form

$$
2 s-2 \leq k \leq 2 s+2 .
$$

On the one hand, these restrictions tell us that for $s>2$, the gravitational interaction, i.e. the case $k=2$, is not allowed. On the other hand, we see that all allowed cubic interactions vertices for graviton and higher spin $s>2$ fields involve higher derivatives, $k>2$.

iii) Restrictions (5.12), (5.13) lead to a surprisingly simple result for the number of allowed parity invariant cubic interaction vertices $p_{[3]}^{-}\left(s^{(1)}, s^{(2)}, s^{(3)} ; k\right)$. Indeed, we see from (5.12) and (5.13) that for spin values $s^{(1)}, s^{(2)}, s^{(3)}$, the integer $k$ takes the values

$$
k=\mathbf{s}, \mathbf{s}-2, \mathbf{s}-4, \ldots, \mathbf{s}-2 s_{\min }, \quad \text { for } d>4 .
$$

This implies that given spin values $s^{(1)}, s^{(2)}, s^{(3)}$, the number of parity invariant cubic interaction vertices $p_{[3]}^{-}\left(s^{(1)}, s^{(2)}, s^{(3)} ; k\right)$ that can be constructed is given by

$$
\mathrm{N}\left(s^{(1)}, s^{(2)}, s^{(3)}\right)=s_{\min }+1, \quad \text { for } d>4 .
$$


iv) Vertices (5.9), with $k$ in (5.15), constitute the complete list of vertices for $d>4$. For $d=4$, the number of allowed vertices is decreased. This is, if $d=4$ then for spin values $s^{(1)}, s^{(2)}, s^{(3)}$, the integer $k$ takes the values 16

$$
k=\mathbf{s}, \mathbf{s}-2 s_{\text {min }}, \quad \text { for } d=4 .
$$

This implies that for spin values $s^{(1)}, s^{(2)}, s^{(3)}$, the number of parity invariant cubic vertices that can be built for massless fields in four dimensions is equal one if $s_{\min }=0$ and two if $s_{\min } \neq 017$.

v) We comment on the relation of our vertices to the vertices for higher spin fields in $A d S$ space [28]-[30]. Clearly, direct comparison of our vertices with AdS vertices is not possible because of two reasons: 1) Our vertices are defined for fields in flat space; 2) Cubic vertices in AdS are given in terms of some explicit generating function, but vertices for three fields with arbitrary (but fixed) spin values are still not available in the literature. Nevertheless, it seems likely that: 1) All our vertices (5.9), (5.15) (and (5.17) for $d=4$ ) allow a smooth extension to $A d S$ space and these vertices, being supplemented by appropriate cosmological constant dependent corrections, coincide with some $A d S$ vertices; 2) The remaining AdS vertices are singular in the flat space limit and do not have flat space counterparts.

Formula (5.9) not only provides a surprisingly simple form for the vertices of massless higher spin fields but also gives a simple form for the vertices of the well-studied massless low spin $s=0,1,2$ fields. By way of example, we consider cubic vertices that describe the self-interaction of spin $s$ field having $k=s$ powers of $\mathbb{P}^{I}$. In the literature, such cubic vertices are referred to as Yang-Mills like interaction vertices. We consider vertices with $s^{(1)}=s^{(2)}=s^{(3)}=s$ and $k=s$ and formula (5.9) leads to

$$
p_{[3]}^{-}(s, s, s ; s)=Z^{s} .
$$

For spin $s=1$ and $s=2$ fields, these vertices describe the respective cubic interaction vertices of Yang-Mills and Einstein theories,

$$
\begin{aligned}
& p_{[3]}^{-}(1,1,1 ; 1)=Z \sim\left(F_{\mu \nu} F^{\mu \nu}\right)_{[3]}, \\
& p_{[3]}^{-}(2,2,2 ; 2)=Z^{2} \sim(\sqrt{g} R)_{[3]},
\end{aligned}
$$

where the subscript [3] of Yang-Mills and Einstein covariant Lagrangians is used to indicate the cubic vertices of these theories. Taking into account the complicated structure of the cubic vertices of Yang-Mills and Einstein theories in covariant approaches, we see that the light-cone gauge approach gives a simpler representation for such vertices. Another attractive property of the lightcone approach is that it allows treating the interaction vertices of Yang-Mills and Einstein theories on an equal footing.

Formula (5.9) provides a convenient representation for other well-known parity invariant cubic interaction vertices of massless low spin fields. These vertices and their Lorentz covariant counterparts are collected in Table I. In Table II, we present light-cone vertices (5.9) involving higher spin fields whose Lorentz covariant descriptions are available in the literature.

\footnotetext{
${ }^{16}$ For $d=4$, the vertices (5.9) with $\mathbf{s}>k>\mathbf{s}-2 s_{\min }$ (see (5.15)) are proportional to $\mathbb{P}^{2}$ (and can be removed by field redefinitions) or to $\alpha_{a}^{I} \alpha_{a}^{I}$ (and do not contribute to the Hamiltonian in view of tracelessness constraint (2.18)). This can be demonstrated straightforwardly using helicity formalism in Ref.[20].

${ }^{17}$ Values of $k$ (5.17) explain the vanishing of the vertices $p_{[3]}^{-}(2,2,2 ; 4)$ (see Table I) and $p_{[3]}^{-}(3,3,3 ; 5)$ (see table II) in $d=4$. The vanishing of the covariant counterpart of our light-cone vertex $p_{[3]}^{-}(3,3,3 ; 5)$ in $d=4$ was discussed in Ref.[39].

${ }^{18}$ Such vertices for spin $s>2$ fields in $4 d$ flat space were built by using light-cone approach in [20]. Our formula (5.18) gives alternative simple expression for these vertices in $d=4$ and provides an extension to arbitarry $d>4$ dimensions on an equal footing.
} 
Table I. Parity invariant cubic vertices for massless low spin $s=0,1,2$ fields. In the 3 rd column, $\phi$ stands for the scalar field, $F_{\mu \nu}$ and $D_{\mu}$ stand for the respective Yang-Mills field strength and covariant derivative $D_{\mu}=\partial_{\mu}+A_{\mu}$, and $R_{\mu \nu \rho \sigma}$ stands for the Riemann tensor 19

\begin{tabular}{|c|c|c|}
\hline $\begin{array}{c}\text { Spin values and } \\
\text { number of derivatives } \\
s^{(1)}, s^{(2)}, s^{(3)} ; k\end{array}$ & $\begin{array}{c}\text { Light-cone } \\
\text { vertex } \\
\left.p_{[3]}^{-} s^{(1)}, s^{(2)}, s^{(3)} ; k\right)\end{array}$ & $\begin{array}{c}\text { Covariant } \\
\text { Lagrangian }\end{array}$ \\
\hline $0,0,0 ; 0$ & 1 & $\phi^{3}$ \\
\hline $0,0,1 ; 1$ & $B^{(3)}$ & $\left(D_{\mu} \phi D^{\mu} \phi\right)_{[3]}$ \\
\hline $0,0,2 ; 2$ & $\left(B^{(3)}\right)^{2}$ & $\left(\sqrt{g} g^{\mu \nu} \partial_{\mu} \phi \partial_{\nu} \phi\right)_{[3]}$ \\
\hline $0,1,1 ; 2$ & $B^{(2)} B^{(3)}$ & $\left(\phi F_{\mu \nu} F^{\mu \nu}\right)_{[3]}$ \\
\hline $0,1,2 ; 3$ & $B^{(2)}\left(B^{(3)}\right)^{2}$ & $\left(\partial^{\mu} \phi F_{\nu \rho} \omega_{\mu}^{\nu \rho}\right)_{[3]}$ \\
\hline $0,2,2 ; 4$ & $\left(B^{(2)} B^{(3)}\right)^{2}$ & $\left(\sqrt{g} \phi R_{\mu \nu \rho \sigma} R^{\mu \nu \rho \sigma}\right)_{[3]}$ \\
\hline $1,1,1 ; 1$ & $Z$ & $\left(F_{\mu \nu} F^{\mu \nu}\right)_{[3]}$ \\
\hline $1,1,1 ; 3$ & $B^{(1)} B^{(2)} B^{(3)}$ & $\left(F_{\mu \nu} F^{\nu \rho} F_{\rho}^{\mu}\right)_{[3]}$ \\
\hline $1,1,2 ; 2$ & $B^{(3)} Z$ & $\left(\sqrt{g} g^{\mu \rho} g^{\nu \sigma} F_{\mu \nu} F_{\rho \sigma}\right)_{[3]}$ \\
\hline $1,1,2 ; 4$ & $B^{(1)} B^{(2)}\left(B^{(3)}\right)^{2}$ & $\left(\sqrt{g} F_{\mu \nu} F_{\rho \sigma} R^{\mu \nu \rho \sigma}\right)_{[3]}$ \\
\hline $1,2,2 ; 3$ & $B^{(2)} B^{(3)} Z$ & $F_{\mu \nu}\left(\omega^{\mu, \rho \sigma} \omega^{\nu}{ }_{\rho \sigma}-\omega^{\rho, \sigma \mu} \omega_{\rho, \sigma}{ }_{[3]}\right.$ \\
\hline $1,2,2 ; 5$ & $B^{(1)}\left(B^{(2)}\right)^{2}\left(B^{(3)}\right)^{2}$ & $\left(F^{\mu \nu} R_{\mu}^{\rho \sigma \lambda} R_{\nu \rho \sigma \lambda}\right)_{[3]}$ \\
\hline $2,2,2 ; 2$ & $Z^{2}$ & $(\sqrt{g} R)_{[3]}$ \\
\hline $2,2,2 ; 4$ & $B^{(1)} B^{(2)} B^{(3)} Z$ & $\left(\sqrt{g} R_{\mu \nu \rho \sigma} R^{\mu \nu \rho \sigma}\right)_{[3]}$ \\
\hline $2,2,2 ; 6$ & $\left(B^{(1)} B^{(2)} B^{(3)}\right)^{2}$ & $\left(\sqrt{g} R_{\rho \sigma}^{\mu \nu} R_{\lambda \tau}^{\rho \sigma} R_{\mu \nu}^{\lambda \tau}\right)_{[3]}$ \\
\hline
\end{tabular}

We note that vertices with $k=\mathbf{s}$ correspond to gauge theory cubic interaction vertices built entirely in terms of gauge field strengths 20 . The vertices with $k<\mathrm{s}$ cannot be built entirely in terms of gauge field strengths. It is the vertices with $k<\mathrm{s}$ that are difficult to construct in Lorentz covariant approaches. The light-cone approach treats all vertices on an equal footing.

We finish with a discussion of the completeness of solution (5.9). Our solution (5.9) provides the complete list of parity invariant cubic vertices for the massless totally symmetric fields in $d \geq 4$ dimensions. In $d>6$ dimensions, so $(d-2)$-invariants constructed out of the antisymmetric LeviCivita symbol $\epsilon^{I_{1} \ldots I_{d-2}}$, the oscillators $\alpha^{(a) I}$, and the momentum $\mathbb{P}^{I}$ are equal to zero, and therefore

\footnotetext{
${ }^{19} \omega_{\mu}^{\nu \rho}$ and $R_{\mu \nu \rho \sigma}$ in covariant vertices $(1,2,2 ; 3),(1,2,2 ; 5)$ stand for the linearized Lorentz connection, $\omega_{\mu}{ }^{\nu \rho}=$ $-\omega_{\mu}{ }^{\rho \nu}$, and the Riemann tensor of the charged (w.r.t. the Yang-Mills gauge group) spin 2 field. These covariant vertices and the vertex $(0,1,2 ; 3)$ are invariant under linearized on-shell gauge transformations.

${ }^{20}$ Our result for the vertex $p_{[3]}^{-}(2,2,2 ; 6)$ implies that there is only one Lorentz covariant $R_{\ldots .}^{3}$ vertex $\left(R_{\ldots} \ldots\right.$ is the Riemann tensor) that gives a non-trivial contribution to the 3-point scattering amplitude. In Ref.[56], it was demonstrated that this is indeed the case.
} 
there are no parity violating cubic vertices for the massless totally symmetric fields. For $d=4,5,6$ there are nontrivial $s o(d-2)$ invariants constructed out of the antisymmetric Levi-Civita symbol. It turns out that for $d=5$, these invariants allow building parity violating cubic vertices for the massless totally symmetric fields21, while for $d=6$, solution 5.9 provides the complete list of cubic vertices for the massless totally symmetric fields (i.e. there are no parity violating cubic vertices for the massless totally symmetric fields in $d=6$ ). The complete list of cubic vertices for massless fields in $d=5,6$ is to be obtained in the respective Sections 8.1 and 8.2 22.

Table II. Parity invariant cubic interaction vertices for massless higher spin fields.

\begin{tabular}{|c|c|c|}
\hline $\begin{array}{c}\text { Spin values and } \\
\text { number of derivatives } \\
s^{(1)}, s^{(2)}, s^{(3)} ; k\end{array}$ & $\begin{array}{c}\text { Light-cone } \\
\text { vertex } \\
p_{[3]}^{-}\left(s^{(1)}, s^{(2)}, s^{(3)} ; k\right)\end{array}$ & $\begin{array}{c}\text { Covariant } \\
\text { Lagrangian }\end{array}$ \\
\hline $2,2,4 ; 4$ & $\left(B^{(3)}\right)^{2} Z^{2}$ & $\mathcal{L}$ (see Ref.[41]) \\
\hline $3,3,3 ; 3$ & $Z^{3}$ & $\mathcal{L}($ see Ref.[37] \\
\hline $3,3,3 ; 5$ & $B^{(1)} B^{(2)} B^{(3)} Z^{2}$ & $\mathcal{L}($ see Ref.[39] $)$ \\
\hline$s, s, s^{\prime} ; k^{\prime}$ & $\left(B^{(1)} B^{(2)}\right)^{s-s_{\min }}\left(B^{(3)}\right)^{s^{\prime}-s_{\min }} Z^{s_{\min }}$ & $\mathcal{L}($ see Refs.[38, [42] $)$ \\
$k^{\prime}=2 s+s^{\prime}-2 s_{\min }$ & & \\
$s_{\min } \equiv \min \left(s, s^{\prime}\right)$ & & \\
\hline
\end{tabular}

\section{Parity invariant cubic interaction vertices for massless and massive fields}

We now study cubic interaction vertices for massless and massive field. We consider cubic vertices for one massive field and two massless fields and cubic vertices for one massless field and two massive fields. In other words, we consider vertices for fields with the following mass values:

$$
\begin{aligned}
& \mathrm{m}_{1}=\mathrm{m}_{2}=0, \quad \mathrm{~m}_{3} \neq 0 \\
& \mathrm{~m}_{1}=\mathrm{m}_{2} \equiv \mathrm{m} \neq 0, \quad \mathrm{~m}_{3}=0 ; \\
& \mathrm{m}_{1} \neq 0, \quad \mathrm{~m}_{2} \neq 0, \quad \mathrm{~m}_{1} \neq \mathrm{m}_{2}, \quad \mathrm{~m}_{3}=0 .
\end{aligned}
$$

We study these cases in turn.

\subsection{Cubic interaction vertices for two massless and one massive fields}

We start with the cubic interaction vertex (4.53) for three fields with the mass values

$$
\mathrm{m}_{1}=\mathrm{m}_{2}=0, \quad \mathrm{~m}_{3} \neq 0
$$

i.e. the massless fields carry external line indices $a=1,2$, while the massive field corresponds to $a=3$. Equations for the vertex involving two massless fields can be obtained from Eqs.(4.48)

\footnotetext{
${ }^{21}$ Complete list of cubic vertices for massless fields in $4 d$ was obtained in [22] and we do not discuss this case.

${ }^{22}$ Complete list of cubic vertices for massless fields in $d=5,6$ was announced in Refs. [53, 54]. In Refs. [53, 54] we mistakenly thought that our solution (5.9) provides the complete list of cubic vertices for massless fields in $d=5$.
} 
in the limit as $m_{1} \rightarrow 0, m_{2} \rightarrow 0$. The general solution for vertex (4.53) is then found to be (see Appendix D)

$$
p_{[3]}^{-}=p_{[3]}^{-}\left(B_{n}^{(3)} ; Q_{m n}^{(a a+1)}, \alpha_{m n}^{(11)}, \alpha_{m n}^{(22)}, Q_{m n}^{(33)}\right),
$$

where we use the notation 23

$$
\begin{aligned}
B_{n}^{(a)} \equiv \frac{\alpha_{n}^{(a) I} \mathbb{P}^{I}}{\beta_{a}}, \quad a=1,2 ; \quad B_{n}^{(3)} \equiv \frac{\alpha_{n}^{(3) I} \mathbb{P}^{I}}{\beta_{3}}+\frac{\check{\beta}_{3}}{2 \beta_{3}} \mathrm{~m}_{3} \alpha_{n}^{(3)}, \\
Q_{m n}^{(12)} \equiv \alpha_{m n}^{(12)}-\frac{2}{\mathrm{~m}_{3}^{2}} B_{m}^{(1)} B_{n}^{(2)} \\
Q_{m n}^{(23)} \equiv \alpha_{m n}^{(23)}+\frac{\alpha_{n}^{(3)}}{\mathrm{m}_{3}} B_{m}^{(2)}+\frac{2}{\mathrm{~m}_{3}^{2}} B_{m}^{(2)} B_{n}^{(3)}, \\
Q_{m n}^{(31)} \equiv \alpha_{m n}^{(31)}-\frac{\alpha_{m}^{(3)}}{\mathrm{m}_{3}} B_{n}^{(1)}+\frac{2}{\mathrm{~m}_{3}^{2}} B_{m}^{(3)} B_{n}^{(1)},
\end{aligned}
$$

and $\alpha_{m n}^{(a b)}, Q_{m n}^{(a a)}$ are defined in (4.38), (4.42). This solution describes cubic interaction vertices for both totally symmetric and mixed-symmetry fields.

We note that all forms in (6.5) that depend on $\mathbb{P}^{I}$ (the linear forms $B_{m}^{(3)}$ and the quadratic forms $Q_{m n}^{(a a+1)}$ ) are non-homogeneous polynomials in $\mathbb{P}^{I}$. Therefore, as seen from (6.5)-(6.9), there is no possibility to construct a cubic vertex that would be a homogeneous polynomial in $\mathbb{P}^{I}$. In other words, the dependence on the linear forms $B_{m}^{(3)}$ and the quadratic forms $Q_{m n}^{(a a+1)}$ leads to the cubic vertices that are non-homogeneous polynomials in $\mathbb{P}^{I}$. The appearance of massive field interaction vertices involving different powers of derivatives is a well-known fact (see e.g. [57, 58]). Thus, we see that the light-cone formalism gives a very simple explanation to this phenomenon by means of the linear forms $B_{m}^{(3)}$ and the quadratic forms $Q_{m n}^{(a a+1)}$. To understand the remaining characteristic properties of solution (6.5), we consider vertices for the totally symmetric fields.

\subsubsection{Cubic interaction vertices for totally symmetric fields}

In this section, we restrict ourselves to cubic interaction vertices for two massless totally symmetric fields and one massive totally symmetric field. To consider the totally symmetric fields, it is sufficient to use one sort of oscillators and we set $\nu=1$ in (6.5)- 6.9). To simplify the formulas we drop the oscillator's subscript $n=1$ and use the simplified notation for oscillators: $\alpha^{I} \equiv \alpha_{1}^{I}$, $\alpha \equiv \alpha_{1}$. The cubic interaction vertex for totally symmetric fields under consideration can then be obtained from the general solution $(6.5)$ by making the identifications

$$
\alpha^{(a) I} \equiv \alpha_{1}^{(a) I}, \quad a=1,2 ; \quad \alpha^{(3) I} \equiv \alpha_{1}^{(3) I} \quad \alpha^{(3)} \equiv \alpha_{1}^{(3)},
$$

in (6.5)-(6.9) and ignoring the contribution of oscillators carrying a subscript $n>1$. Adopting simplified notation (6.10) for forms (6.6)- (6.9):

$$
B^{(a)} \equiv B_{1}^{(a)}, \quad Q^{(a b)} \equiv Q_{11}^{(a b)}, \quad \alpha^{(a b)} \equiv \alpha_{11}^{(a b)},
$$

we see that vertex (6.5) takes the form

$$
p_{[3]}^{-}=p_{[3]}^{-}\left(B^{(3)} ; Q^{(a a+1)}, \alpha^{(11)}, \alpha^{(22)}, Q^{(33)}\right) .
$$

\footnotetext{
${ }^{23}$ We recall that the short notation like $p_{[3]}^{-}\left(Q^{(a a+1)}\right)$ is used to indicate a dependence of $p_{[3]}^{-}$on $Q^{(12)}, Q^{(23)}, Q^{(31)}$.
} 
Vertex (6.12) describes the interaction of the towers of massive and massless fields (2.19), (2.20). We now obtain vertex for two massless totally symmetric spin $s^{(1)}, s^{(2)}$ fields and one massive totally symmetric spin $s^{(3)}$ field. The massless totally symmetric spin $s^{(1)}$ and $s^{(2)}$ fields are described by respective ket-vectors $\left|\phi_{s^{(1)}}^{\mathrm{m}_{1}=0}\right\rangle$ and $\left|\phi_{s^{(2)}}^{\mathrm{m}_{2}=0}\right\rangle$, while the massive totally symmetric spin $s^{(3)}$ field is described by a ket-vector $\left|\phi_{s(3)}\right\rangle$. The ket-vectors of massless fields $\left|\phi_{s(a)}^{\mathrm{m}_{a}=0}\right\rangle, a=1,2$, can be obtained from (2.15) by the replacement $s \rightarrow s^{(a)}, \alpha^{I} \rightarrow \alpha^{(a) I}, a=1,2$, in (2.15), while the ket-vector of the massive field $\left|\phi_{s}(3)\right\rangle$ can be obtained from (2.14) by the replacement $s \rightarrow s^{(3)}$, $\alpha^{I} \rightarrow \alpha^{(3) I}, \alpha \rightarrow \alpha^{(3)}$ in (2.14). Taking into account that the ket-vectors $\left|\phi_{s}^{\mathrm{m}_{a}=0}\right\rangle, a=1,2$, are the respective degree $s^{(a)}$ homogeneous polynomials in the oscillators $\alpha^{(a) I}$ (see (2.17)), while the ket-vector $\left|\phi_{s^{(3)}}\right\rangle$ is a degree $s^{(3)}$ homogeneous polynomial in the oscillators $\alpha^{(3) I}, \alpha^{(3)}$ (see (2.16) ) it is easy to understand that the vertex we are interested in must satisfy the equations

$$
\begin{aligned}
& \left(\alpha^{(a) I} \bar{\alpha}^{(a) I}-s^{(a)}\right)\left|p_{[3]}^{-}\right\rangle=0, \quad a=1,2, \\
& \left(\alpha^{(3) I} \bar{\alpha}^{(3) I}+\alpha^{(3)} \bar{\alpha}^{(3)}-s^{(3)}\right)\left|p_{[3]}^{-}\right\rangle=0 .
\end{aligned}
$$

These equations tell us that the vertex must be a degree $s^{(a)}$ homogeneous polynomial in the respective oscillators. Taking into account that the forms $B^{(3)}$ and $Q^{(a a+1)}$ are the respective degree 1 and 2 homogeneous polynomials in the oscillators we find the general solution of Eqs. (6.13), (6.14) as 24

$$
p_{[3]}^{-}\left(s^{(1)}, s^{(2)}, s^{(3)} ; x\right)=\left(B^{(3)}\right)^{x} \prod_{a=1}^{3}\left(Q^{(a a+1)}\right)^{y^{(a+2)}},
$$

where integers $y^{(a)}$ are expressible in terms of the $s^{(a)}$ and an integer $x$ by the relations

$$
\begin{aligned}
& y^{(a)}=\frac{\mathbf{s}-x}{2}-s^{(a)}, \quad a=1,2, \\
& y^{(3)}=\frac{\mathbf{s}+x}{2}-s^{(3)} .
\end{aligned}
$$

The integer $x$ expresses the freedom of the solution and labels all possible cubic interaction vertices that can be constructed for the fields under consideration. For vertex (6.15) to be sensible, we impose the restrictions

$$
\begin{aligned}
& x \geq 0, \quad y^{(a)} \geq 0, \quad a=1,2,3 ; \\
& \mathbf{s}-x \quad \text { even integer, }
\end{aligned}
$$

which amount to the requirement that the powers of all forms in (6.15) be non-negative integers. We note that using relations (6.16), (6.17) allows rewriting the restrictions (6.18) as

$$
\max \left(0, s^{(3)}-s^{(1)}-s^{(2)}\right) \leq x \leq s^{(3)}-\left|s^{(1)}-s^{(2)}\right| .
$$

Compared to the vertex for three massless fields (5.9), vertex (6.15) is a non-homogeneous polynomial in $\mathbb{P}^{I}$. An interesting property of vertex (6.15) is that the maximal number of powers of the momentum $\mathbb{P}^{I}$, denoted by $k_{\max }$, is independent of $x$ and is determined only by $\mathbf{s} 26$

$$
k_{\max }=\mathbf{s} .
$$

\footnotetext{
${ }^{24}$ We ignore the contribution of $\alpha^{(11)}-, \alpha^{(22)}-, Q^{(33)}$-terms of 6.12) to vertex 6.15). Because of the tracelessness constraints 2.18 the contribution of these terms to the Hamiltonian $P_{[3]}^{-}(3.3)$ vanishes.

${ }^{25}$ If $x=0$, then restrictions (6.20) become the restrictions well known in the angular momentum theory: $\mid s^{(1)}-$ $s^{(2)} \mid \leq s^{(3)} \leq s^{(1)}+s^{(2)}$.

${ }^{26}$ Expressions for $B^{(3)}$ and $Q^{(a a+1)}$ 6.6) 6.16, 6.17) into account we find 6.21).
} 


\subsection{Cubic interaction vertices for one massless and two massive fields with the same mass values}

The case under consideration is most interesting because it involves the minimal Yang-Mills and gravitational interactions of massive arbitrary spin fields as particular cases. We now consider the cubic interaction vertex (4.53) for one massless field and two massive fields with the same mass values,

$$
\mathrm{m}_{1}=\mathrm{m}_{2} \equiv \mathrm{m} \neq 0, \quad \mathrm{~m}_{3}=0
$$

i.e. the massive fields carry external line indices $a=1,2$, while the massless field corresponds to $a=3$. The analysis of equations for the vertex is straightforward and the general solution is found to be (see Appendix D)

$$
p_{[3]}^{-}=p_{[3]}^{-}\left(L_{n}^{(1)}, L_{n}^{(2)}, B_{n}^{(3)} ; Q_{m n}^{(12)}, Q_{m n}^{(11)}, Q_{m n}^{(22)}, \alpha_{m n}^{(33)} ; Z_{m n q}\right)
$$

where we use the notation

$$
\begin{aligned}
& L_{n}^{(1)} \equiv B_{n}^{(1)}+\frac{1}{2} \mathrm{~m} \alpha_{n}^{(1)}, \quad L_{n}^{(2)} \equiv B_{n}^{(2)}-\frac{1}{2} \mathrm{~m} \alpha_{n}^{(2)}, \\
& B_{n}^{(a)} \equiv \frac{\alpha_{n}^{(a) I} \mathbb{P}^{I}}{\beta_{a}}+\frac{\check{\beta}_{a}}{2 \beta_{a}} \mathrm{~m} \alpha_{n}^{(a)}, \quad a=1,2 ; \\
& B_{n}^{(3)} \equiv \frac{\alpha_{n}^{(3) I} \mathbb{P}^{I}}{\beta_{3}} \\
& Q_{m n}^{(12)} \equiv \alpha_{m n}^{(12)}+\frac{\alpha_{n}^{(2)}}{\mathrm{m}} B_{m}^{(1)}-\frac{\alpha_{m}^{(1)}}{\mathrm{m}} B_{n}^{(2)}, \\
& Z_{m n q} \equiv L_{m}^{(1)} \alpha_{n q}^{(23)}+L_{n}^{(2)} \alpha_{q m}^{(31)}+B_{q}^{(3)}\left(\alpha_{m n}^{(12)}-\alpha_{m}^{(1)} \alpha_{n}^{(2)}\right),
\end{aligned}
$$

and $\alpha_{m n}^{(a b)}, Q_{m n}^{(a a)}$ are defined in (4.38), 4.42). Thus, we see that vertex (6.23) depends, among other things, on linear forms $B_{n}^{(3)}$ (6.26), which are degree 1 homogeneous polynomials in the momentum $\mathbb{P}^{I}$. This implies that cubic interaction vertices that are homogeneous polynomials in $\mathbb{P}^{I}$ can be constructed for certain fields. This also implies that the minimal number of powers of $\mathbb{P}^{I}$ in (6.23) is not equal to zero in general (for example, the dependence on $B_{m}^{(3)}$ leads to an increasing number of powers of the momentum $\mathbb{P}^{I}$ ). All the remaining forms that depend on the momentum $\mathbb{P}^{I}$ and enter the cubic vertex (the linear forms $L_{n}^{(1)}, L_{n}^{(2)}$ and the quadratic forms $Q_{m n}^{(12)}$ ) are nonhomogeneous polynomials in $\mathbb{P}^{I}$. To discuss the remaining important properties of solution (6.23) we restrict attention to cubic vertices for the totally symmetric fields.

\subsubsection{Cubic interaction vertices for totally symmetric fields}

In this section, we restrict ourselves to cubic interaction vertices for the totally symmetric fields with mass values given in (6.22). As usual, we use one sort of oscillators, i.e. we set $\nu=1$ in (6.23)-(6.28) and simplify formulas by dropping the oscillator's subscript $n=1: \alpha^{I} \equiv \alpha_{1}^{I}$, $\alpha \equiv \alpha_{1}$. The cubic interaction vertex for the totally symmetric fields under consideration can be obtained from the general solution (6.23) by making the identifications

$$
\alpha^{(a) I} \equiv \alpha_{1}^{(a) I}, \quad \alpha^{(a)} \equiv \alpha_{1}^{(a)}, \quad a=1,2 ; \quad \alpha^{(3) I} \equiv \alpha_{1}^{(3) I},
$$


in (6.23)-(6.28) and ignoring the contribution of oscillators carrying a subscript $n>1$. Adopting the simplified notation (6.29) for forms (6.24)- (6.28),

$$
L^{(a)} \equiv L_{1}^{(a)}, \quad B^{(a)} \equiv B_{1}^{(a)}, \quad \alpha^{(a b)} \equiv \alpha_{11}^{(a b)}, \quad Q^{(a b)} \equiv Q_{11}^{(a b)}, \quad Z \equiv Z_{111}
$$

we see that vertex (6.23) takes the form

$$
p_{[3]}^{-}=p_{[3]}^{-}\left(L^{(1)}, L^{(2)}, B^{(3)} ; Q^{(12)}, Q^{(11)}, Q^{(22)}, \alpha^{(33)} ; Z\right) .
$$

Vertex (6.31) describes the interaction of the towers of massive and massless fields (2.19), (2.20). We next obtain the vertex for two massive totally symmetric spin $s^{(1)}, s^{(2)}$ fields and one massless totally symmetric spin $s^{(3)}$ field. Two massive totally symmetric spin $s^{(1)}$ and $s^{(2)}$ fields are described by the respective ket-vectors $\left|\phi_{s^{(1)}}\right\rangle$ and $\left|\phi_{s^{(2)}}\right\rangle$, while one massless totally symmetric spin $s^{(3)}$ field is described by a ket-vector $\left|\phi_{s^{(3)}}^{\mathrm{m}_{3}=0}\right\rangle$. The ket-vectors of massive fields $\left|\phi_{s^{(a)}}\right\rangle, a=1,2$, can be obtained from (2.14) by the replacement $s \rightarrow s^{(a)}, \alpha^{I} \rightarrow \alpha^{(a) I}, \alpha \rightarrow \alpha^{(a)}, a=1,2$, in (2.14), while the ket-vector of massless field $\left|\phi_{s^{(3)}}^{\mathrm{m}_{3}=0}\right\rangle$ can be obtained from (2.15) by the replacement $s \rightarrow s^{(3)}, \alpha^{I} \rightarrow \alpha^{(3) I}$ in (2.15). Taking into account that the ket-vectors $\left|\phi_{s^{(a)}}\right\rangle, a=1,2$, are the respective degree $s^{(a)}$ homogeneous polynomials in the oscillators $\alpha^{(a) I}, \alpha^{(a)}$ (see (2.16)), while the ket-vector $\left|\phi_{s^{(3)}}^{\mathrm{m}_{3}=0}\right\rangle$ is a degree $s^{(3)}$ homogeneous polynomial in the oscillator $\alpha^{(3) I}$ (see (2.17)) it is easy to understand that the vertex we are interested in must satisfy the equations

$$
\begin{aligned}
& \left(\alpha^{(a) I} \bar{\alpha}^{(a) I}+\alpha^{(a)} \bar{\alpha}^{(a)}-s^{(a)}\right)\left|p_{[3]}^{-}\right\rangle=0, \quad a=1,2, \\
& \left(\alpha^{(3) I} \bar{\alpha}^{(3) I}-s^{(3)}\right)\left|p_{[3]}^{-}\right\rangle=0 .
\end{aligned}
$$

These equations tell us that the vertex must be a degree $s^{(a)}$ homogeneous polynomial in the respective oscillators. Taking into account that the forms $L^{(1)}, L^{(2)}, B^{(3)}$ (6.30) are degree 1 homogeneous polynomials in the oscillators, while the forms $Q^{(12)}$ and $Z$ (6.30) are respective degree 2 and 3 homogeneous polynomials in the oscillators we find the general solution of Eqs. (6.32), (6.33) as 27

$$
p_{[3]}^{-}\left(s^{(1)}, s^{(2)}, s^{(3)} ; k_{\min }, k_{\max }\right)=\left(L^{(1)}\right)^{x^{(1)}}\left(L^{(2)}\right)^{x^{(2)}}\left(B^{(3)}\right)^{x^{(3)}}\left(Q^{(12)}\right)^{y^{(3)}} Z^{y},
$$

where the parameters $x^{(1)}, x^{(2)}, x^{(3)}, y^{(3)}, y$ are given by

$$
\begin{aligned}
& x^{(1)}=k_{\text {max }}-k_{\text {min }}-s^{(2)}, \\
& x^{(2)}=k_{\text {max }}-k_{\text {min }}-s^{(1)}, \\
& x^{(3)}=k_{\text {min }} \\
& y^{(3)}=\mathbf{s}-2 s^{(3)}-k_{\max }+2 k_{\text {min }}, \\
& y=s^{(3)}-k_{\text {min }}
\end{aligned}
$$

and $\mathbf{s}$ is defined in (5.10). New integers $k_{\min }$ and $k_{\max }$ in (6.34)-6.39) are the freedom in our solution. In general, vertex (6.34) is a non-homogeneous polynomial in the momentum $\mathbb{P}^{I}$ and the integers $k_{\min }$ and $k_{\max }$ are the respective minimal and maximal numbers of powers of the

\footnotetext{
${ }^{27}$ We ignore the contribution of $Q^{(11)}-, Q^{(22)}-, \alpha^{(33)}$-terms of 6.31) to vertex 6.34). Because of the tracelessness constraints (2.18) the contribution of these terms to the Hamiltonian $P_{[3]}^{-}(3.3)$ vanishes.
} 
momentum $\mathbb{P}^{I}$ in 6.34 . As noted above, the minimal number of powers of the momentum $\mathbb{P}^{I}$ is not equal to zero in general. For vertex (6.34) to be sensible, we should impose the restrictions

$$
\begin{array}{ll}
x^{(a)} \geq 0, & a=1,2,3 ; \\
y^{(3)} \geq 0, & y \geq 0,
\end{array}
$$

which amount to requiring the powers of all forms in (6.34) to be non-negative integers. With (6.35)-(6.39), restrictions (6.40), (6.41) can be rewritten in a more convenient form as

$$
\begin{aligned}
k_{\min }+\max _{a=1,2} s^{(a)} & \leq k_{\max } \leq \mathbf{s}-2 s^{(3)}+2 k_{\text {min }}, \\
0 & \leq k_{\min } \leq s^{(3)} .
\end{aligned}
$$

\subsubsection{Minimal Yang-Mills interaction of massive totally symmetric arbitrary spin field}

We now apply our results in Section 6.2.1 to the discussion of the minimal Yang-Mills interaction of the massive totally symmetric arbitrary spin field. We first present the list of all cubic vertices for the massive totally symmetric spin $s$ field interacting with the massless spin 1 field (Yang-Mills field). This is, we consider the vertices (6.34) with the spin values

$$
s^{(1)}=s^{(2)}=s, \quad s^{(3)}=1 .
$$

Restrictions (6.43) lead to two allowed values of $k_{\min }: k_{\min }=0,1$. Substituting these values of $k_{\min }$ in 6.42), we obtain two families of vertices

$$
\begin{array}{lll}
k_{\text {min }}=1, & s+1 \leq k_{\max } \leq 2 s+1, & s \geq 0 ; \\
k_{\text {min }}=0, & s \leq k_{\max } \leq 2 s-1, & s \geq 1 .
\end{array}
$$

We now discuss those vertices from the list in (6.45), (6.46) that correspond to the minimal YangMills interaction of the massive arbitrary spin field. We consider various spin fields in turn.

a) Spin $s=0$ field. The vertices for the spin $s=0$ field fall in the family of vertices given in (6.45). Plugging $s=0$ in (6.45) we obtain $k_{\text {min }}=k_{\max }=1$ and therefore the cubic vertex of the minimal Yang-Mills interaction of the massive scalar field is a degree 1 homogeneous polynomial in derivatives. Relations (6.34)-(6.39) lead to the minimal Yang-Mills interaction of the massive scalar field

$$
p_{[3]}^{-}(0,0,1 ; 1,1)=B^{(3)} .
$$

b) Spin $s \geq 1$ field. All vertices given in (6.45), 6.46) are candidates for the minimal Yang-Mills interaction of the spin $s \geq 1$ field. We therefore impose an additional requirement, which allows us to choose one suitable vertex: given spin $s$, we look for the vertex with the minimal value of $k_{\max }$. It can be seen that such a vertex is given by (6.46) with $k_{\max }=s$. The choice of the vertex from (6.46) implies $k_{\text {min }}=0$ and we obtain from (6.34)-(6.39) the minimal Yang-Mills interaction of the massive spin $s \geq 1$ field 29 ,

$$
p_{[3]}^{-}(s, s, 1 ; 0, s)=\left(Q^{(12)}\right)^{s-1} Z, \quad s \geq 1 .
$$

\footnotetext{
${ }^{28}$ This can be checked by taking into account that the forms $L^{(1)}, L^{(2)}, Q^{(12)}$ and $Z$ are degree 1 polynomials in $\mathbb{P}^{I}$, while the form $B^{(3)}$ is degree 1 homogeneous polynomial in $\mathbb{P}^{I}$ (see (6.24)- 6.28).

${ }^{29}$ A gauge invariant description of the electromagnetic interaction of the massive spin $s=2$ field was obtained in [60]. The application of the approach in [60] to the massive arbitrary spin $s$ field can be found in [61]. The derivation of the electromagnetic interaction of massive spin $s=2,3$ fields from string theory is given in [62, 63]. In these references, the electromagnetic field is treated as an external (non-dynamical) field.
} 
A few remarks are in order.

i) The forms $B^{(3)}(6.24)$ and $Z$ (6.28) have smooth massless limit $(\mathrm{m} \rightarrow 0)$. Therefore, the minimal Yang-Mills interactions of the massive low spin $s=0,1$ fields given in (6.47), (6.48) have a smooth massless limit, as they should. These interactions in the massless limit coincide with the respective interactions of the massless spin $s=0,1$ fields in Table I.

ii) The form $Q^{(12)}$ (6.27) does not have a smooth massless limit $(\mathrm{m} \rightarrow 0)$. This implies that the minimal Yang-Mills interaction of the massive spin $s>1$ field (6.48) does not admit a sensible massless limit; in light-cone approach, it is contribution of $Q^{(12)}$ that explains why the minimal Yang-Mills interaction of the massive spin $s>1$ field does not admit the massless limit. As was expected, the minimal Yang-Mills interaction of the massive spin $s>1$ field (6.48) involves higher derivatives. The appearance of the higher derivatives in (6.48) can be seen from the expression for $Q^{(12)}$ (6.27).

\subsubsection{Gravitational interaction of massive totally symmetric arbitrary spin field}

We proceed with the discussion of the gravitational interaction of the massive totally symmetric arbitrary spin field. We first present the list of all cubic vertices for the massive totally symmetric spin $s$ field interacting with the massless spin 2 field. This is, we consider vertices (6.34) with the spin values

$$
s^{(1)}=s^{(2)}=s, \quad s^{(3)}=2 .
$$

Restrictions (6.43) lead to three allowed values of $k_{\min }: k_{\min }=0,1,2$. Plugging these values of $k_{\text {min }}$ in restrictions (6.42), we obtain three families of vertices

$$
\begin{array}{lll}
k_{\text {min }}=2, & s+2 \leq k_{\text {max }} \leq 2 s+2, & s \geq 0 ; \\
k_{\text {min }}=1, & s+1 \leq k_{\text {max }} \leq 2 s, & s \geq 1 ; \\
k_{\text {min }}=0, & s \leq k_{\text {max }} \leq 2 s-2, & s \geq 2 .
\end{array}
$$

We now discuss those vertices from the list given in 6.50) -6.52) that correspond to the gravitational interaction of the massive arbitrary spin field. We consider various spin fields in turn.

a) Spin $s=0$ field. The gravitational interaction of the massive scalar field is given by (6.50). Plugging $s=0$ in (6.50), we obtain the well-known relation $k_{\min }=k_{\max }=2$, which tells us that the cubic vertex of the gravitational interaction of the massive scalar field is a degree 2 homogeneous polynomial in the derivatives. Formulas 6.34) (6.39) lead to the gravitational interaction of the massive scalar field,

$$
p_{[3]}^{-}(0,0,2 ; 2,2)=\left(B^{(3)}\right)^{2} .
$$

b) Spin $s=1$ field. The obvious candidates for the gravitational interaction vertices of the massive vector field are given in (6.50), (6.51). If $s=1$, then restrictions (6.50) lead to $3 \leq k_{\max } \leq 4$, and therefore vertices (6.50) involve higher derivatives. But from the covariant approach, it is well known that the gravitational interaction of the massive vector field does not involve higher derivatives. We therefore restrict attention to the vertices given in (6.51). Plugging $s=1$ in (6.51) we obtain $k_{\max }=2$. Formulas (6.34)-(6.39) then lead to the gravitational interaction of the massive vector field

$$
p_{[3]}^{-}(1,1,2 ; 1,2)=B^{(3)} Z \text {. }
$$

c) Spin $s \geq 2$ field. All vertices given in (6.50)- 6.52 are candidates for the gravitational interaction of spin $s \geq 2$ field. We should impose some additional requirement that would allow 
us to choose one suitable vertex. Our additional requirement is that given a spin $s$, we look for vertex with the minimal value of $k_{\max }$. It can be seen that such a vertex is given by (6.52) with $k_{\max }=s$. We note that $k_{\min }=0$ and relations (6.34)-(6.39) lead to the gravitational interaction of the massive spin $s \geq 2$ field,

$$
p_{[3]}^{-}(s, s, 2 ; 0, s)=\left(Q^{(12)}\right)^{s-2} Z^{2}, \quad s \geq 2 .
$$

A few remarks are in order.

i) Since the forms $B^{(3)}(6.24)$ and $Z(6.28)$ have a smooth massless limit $(\mathrm{m} \rightarrow 0)$, the gravitational interactions of the massive low spin $s=0,1,2$ fields (6.53)-(6.55) have smooth massless limit, as they should. These gravitational interactions in the massless limit reduce to the corresponding interactions of the massless spin $s=0,1,2$ fields given in Table I.

ii) Since the form $Q^{(12)}$ 6.27) does not have a smooth massless limit $(\mathrm{m} \rightarrow 0)$, the gravitational interaction of the massive higher spin $s>2$ field (6.55) does not admit a sensible massless limit; it is the form $Q^{(12)}$ that explains why the gravitational interaction of the massive higher spin field does not admit the massless limit. Higher derivatives in the gravitational interaction of the massive higher spin field are related to the contribution of $Q^{(12)}(6.27)$.

\subsection{Cubic interaction vertices for one massless and two massive fields with different mass values}

We now consider the cubic interaction vertex (4.53) for fields with the following mass values:

$$
\mathrm{m}_{1} \neq 0, \quad \mathrm{~m}_{2} \neq 0, \quad \mathrm{~m}_{1} \neq \mathrm{m}_{2}, \quad \mathrm{~m}_{3}=0,
$$

i.e. the massive fields carry external line indices $a=1,2$, while the massless field corresponds to $a=3$. Equations for the vertex involving one massless field can be obtained from Eqs. (4.48) in the limit as $\mathrm{m}_{3} \rightarrow 0$. The general solution for vertex (4.53) then takes the form (see Appendix D)

$$
p_{[3]}^{-}=p_{[3]}^{-}\left(L_{n}^{(1)}, L_{n}^{(2)} ; Q_{m n}^{(a a+1)}, Q_{m n}^{(11)}, Q_{m n}^{(22)}, \alpha_{m n}^{(33)}\right),
$$

where we use the notation

$$
\begin{gathered}
L_{n}^{(1)} \equiv B_{n}^{(1)}+\frac{\mathrm{m}_{2}^{2}}{2 \mathrm{~m}_{1}} \alpha_{n}^{(1)}, \quad L_{n}^{(2)} \equiv B_{n}^{(2)}-\frac{\mathrm{m}_{1}^{2}}{2 \mathrm{~m}_{2}} \alpha_{n}^{(2)} \\
B_{n}^{(a)} \equiv \frac{\alpha_{n}^{(a) I} \mathbb{P}^{I}}{\beta_{a}}+\frac{\check{\beta}_{a}}{2 \beta_{a}} \mathrm{~m}_{a} \alpha_{n}^{(a)}, \quad a=1,2 \\
B_{n}^{(3)} \equiv \frac{\alpha_{n}^{(3) I} \mathbb{P}^{I}}{\beta_{3}}, \\
Q_{m n}^{(12)} \equiv \alpha_{m n}^{(12)}+\frac{\alpha_{n}^{(2)}}{\mathrm{m}_{2}} B_{m}^{(1)}-\frac{\alpha_{m}^{(1)}}{\mathrm{m}_{1}} B_{n}^{(2)}, \\
Q_{m n}^{(23)} \equiv \alpha_{m n}^{(23)}+\frac{\mathrm{m}_{2} \alpha_{m}^{(2)}}{\mathrm{m}_{1}^{2}-\mathrm{m}_{2}^{2}} B_{n}^{(3)}-\frac{2}{\mathrm{~m}_{1}^{2}-\mathrm{m}_{2}^{2}} B_{m}^{(2)} B_{n}^{(3)} \\
Q_{m n}^{(31)} \equiv \alpha_{m n}^{(31)}+\frac{\mathrm{m}_{1} \alpha_{n}^{(1)}}{\mathrm{m}_{1}^{2}-\mathrm{m}_{2}^{2}} B_{m}^{(3)}+\frac{2}{\mathrm{~m}_{1}^{2}-\mathrm{m}_{2}^{2}} B_{m}^{(3)} B_{n}^{(1)}
\end{gathered}
$$

\footnotetext{
${ }^{30}$ Gauge invariant formulations of the gravitational interaction of massive fields are studied e.g. in [64, 65]. Interesting discussion of various aspects of the massive spin 2 field in gravitational background may be found in [66, 67].
} 
and $\alpha_{m n}^{(a b)}, Q_{m n}^{(a a)}$ are defined in (4.38), (4.42). An interesting property of the solution obtained is the appearance of expressions like $\mathrm{m}_{1}^{2}-\mathrm{m}_{2}^{2}$ in the denominators of the quadratic forms $Q^{(23)}(6.62)$ and $Q^{(31)}$ 6.63); the forms $Q^{(23)}, Q^{(31)}$ are therefore singular as $\mathrm{m}_{1} \rightarrow \mathrm{m}_{2}$. For this reason, we considered the case of $\mathrm{m}_{1}=\mathrm{m}_{2}$ separately in Section 6.2.

As can be seen from (6.57)-6.63), it is impossible to construct a cubic vertex that would be a homogeneous polynomial in the momentum $\mathbb{P}^{I}$. All forms that depend on $\mathbb{P}^{I}$ and enter the vertex (i.e. $L_{n}^{(1)}, L_{n}^{(2)}$, and $Q_{m n}^{(a a+1)}$ ) are non-homogeneous polynomials in $\mathbb{P}^{I}$. This implies that the cubic vertex is a non-homogeneous polynomial in $\mathbb{P}^{I}$ in general. To understand the remaining characteristic properties of solution (6.57), we consider the vertices for the totally symmetric fields.

\subsubsection{Cubic interaction vertices for totally symmetric fields}

The discussion of cubic interaction vertices for two massive totally symmetric fields with different mass values and one massless totally symmetric field largely follows that in Section 6.2.1. The cubic vertex we are interested in can be obtained from the general solution (6.57) by making identifications 6.29) in 6.57)-6.63) and ignoring the contribution of oscillators carrying a subscript $n>1$. From (6.57), adopting the simplified notation for forms (6.58)-(6.63):

$$
L^{(a)} \equiv L_{1}^{(a)}, \quad B^{(a)} \equiv B_{1}^{(a)}, \quad Q^{(a b)} \equiv Q_{11}^{(a b)}, \quad \alpha^{(a b)} \equiv \alpha_{11}^{(a b)},
$$

we obtain the vertex that describes the interaction of towers of massive and massless totally symmetric fields

$$
p_{[3]}^{-}=p_{[3]}^{-}\left(L^{(1)}, L^{(2)} ; Q^{(a a+1)}, Q^{(11)}, Q^{(22)}, \alpha^{(33)}\right) .
$$

The vertices for two massive totally symmetric spin $s^{(1)}, s^{(2)}$ fields $\left|\phi_{s^{(1)}}\right\rangle,\left|\phi_{s^{(2)}}\right\rangle$ with different mass values and one massless totally symmetric spin $s^{(3)}$ field $\left|\phi_{s^{(3)}}^{\mathrm{m}_{3}=0}\right\rangle$ can be obtained by solving Eqs. 6.32), 6.33) with $p_{[3]}^{-}$given in (6.65). We then obtain the cubic vertex 31

$$
p_{[3]}^{-}\left(s^{(1)}, s^{(2)}, s^{(3)} ; x^{(1)}, x^{(2)}\right)=\left(L^{(1)}\right)^{x^{(1)}}\left(L^{(2)}\right)^{x^{(2)}}\left(Q^{(12)}\right)^{y^{(3)}}\left(Q^{(23)}\right)^{y^{(1)}}\left(Q^{(31)}\right)^{y^{(2)}},
$$

where the parameters $y^{(a)}$ are given by

$$
\begin{aligned}
& y^{(1)}=\frac{1}{2}\left(s^{(2)}+s^{(3)}-s^{(1)}+x^{(1)}-x^{(2)}\right), \\
& y^{(2)}=\frac{1}{2}\left(s^{(1)}+s^{(3)}-s^{(2)}-x^{(1)}+x^{(2)}\right), \\
& y^{(3)}=\frac{1}{2}\left(s^{(1)}+s^{(2)}-s^{(3)}-x^{(1)}-x^{(2)}\right) .
\end{aligned}
$$

Two integers $x^{(1)}, x^{(2)}$ are the freedom of our solution. For fixed spin values $s^{(1)}, s^{(2)}, s^{(3)}$, these integers label all possible cubic interaction vertices that can be built for the fields under consideration. For vertex (6.66) to be sensible we impose the restrictions

$$
\begin{aligned}
& y^{(a)} \geq 0, \quad a=1,2,3 \\
& x^{(1)} \geq 0, \quad x^{(2)} \geq 0, \\
& \mathbf{s}-x^{(1)}-x^{(2)} \quad \text { even integer },
\end{aligned}
$$

\footnotetext{
${ }^{31}$ We ignore the contribution of $Q^{(11)}-, Q^{(22)}-, \alpha^{(33)}$-terms of 6.65 to vertex 6.66). Because of the tracelessness constraints 2.18), the contribution of these terms to the Hamiltonian $P_{[3]}^{-}(3.3)$ vanishes.
} 
which amount to the requirement that the powers of all forms in (6.66) be non-negative integers. The maximal number of powers of $\mathbb{P}^{I}$ in $(6.66)$, which is denoted by $k_{\max }$, is given by 32

$$
k_{\max }=\frac{1}{2}\left(s^{(1)}+s^{(2)}+3 s^{(3)}+x^{(1)}+x^{(2)}\right) .
$$

We note that using (6.67)- (6.69) allows rewriting restrictions 6.70) in the equivalent form 33

$$
\left|s^{(1)}-s^{(2)}-x^{(1)}+x^{(2)}\right| \leq s^{(3)} \leq s^{(1)}+s^{(2)}-x^{(1)}-x^{(2)} .
$$

\section{Parity invariant cubic interaction vertices for massive fields}

We finally consider the cubic interaction vertex (4.53) for three massive fields:

$$
\mathrm{m}_{1} \neq 0, \quad \mathrm{~m}_{2} \neq 0, \quad \mathrm{~m}_{3} \neq 0 .
$$

The general solution for vertex (4.53) is found to be (see Appendix D)

$$
p_{[3]}^{-}=p_{[3]}^{-}\left(L_{n}^{(a)} ; Q_{m n}^{(a a+1)}, Q_{m n}^{(a a)}\right),
$$

where we use the notation

$$
\begin{aligned}
& L_{n}^{(a)} \equiv B_{n}^{(a)}+\frac{\mathrm{m}_{a+1}^{2}-\mathrm{m}_{a+2}^{2}}{2 \mathrm{~m}_{a}} \alpha_{n}^{(a)}, \quad B_{n}^{(a)} \equiv \frac{\alpha_{n}^{(a) I} \mathbb{P}^{I}}{\beta_{a}}+\frac{\check{\beta}_{a}}{2 \beta_{a}} \mathrm{~m}_{a} \alpha_{n}^{(a)}, \\
& Q_{m n}^{(a a+1)} \equiv \alpha_{m n}^{(a a+1)}+\frac{\alpha_{n}^{(a+1)}}{\mathrm{m}_{a+1}} B_{m}^{(a)}-\frac{\alpha_{m}^{(a)}}{\mathrm{m}_{a}} B_{n}^{(a+1)}-\frac{\mathrm{m}_{a+2}^{2}}{2 \mathrm{~m}_{a} \mathrm{~m}_{a+1}} \alpha_{m}^{(a)} \alpha_{n}^{(a+1)},
\end{aligned}
$$

and $\alpha_{m n}^{(a b)}, Q_{m n}^{(a a)}$ are defined in (4.38), (4.42). From the expressions for the quadratic forms $Q_{m n}^{(a a+1)}$ (7.4), it follows that the cubic vertex for massive fields is singular as $\mathrm{m}_{a} \rightarrow 0, a=1,2,3$. The remaining quadratic forms $Q_{m n}^{(a a)}$ do not contribute to the Hamiltonian when the ket-vectors $\left|\phi_{a}\right\rangle$ are restricted to be traceless. We note, however, that it is sometimes convenient to formulate interacting fields theories in terms of ket-vectors that are not subjected to the tracelessness constraint. For example, the ket-vectors of the light-cone gauge string field theories are not subjected to the tracelessness constraint. We now restrict attention to vertices for the totally symmetric fields.

\subsection{Cubic interaction vertices for totally symmetric fields}

To obtain the cubic interaction vertex for the massive totally symmetric fields we simply set $\nu=1$ in relations (7.2)-(7.4). To simplify the formulas we drop the oscillator's subscript $n=1$ and use the simplified notation $\alpha^{I}=\alpha_{1}^{I}, \alpha=\alpha_{1}$. The expression for the cubic vertex can then be obtained from the general solution (7.2) by using the identifications

$$
\alpha^{(a) I} \equiv \alpha_{1}^{(a) I}, \quad \alpha^{(a)} \equiv \alpha_{1}^{(a)}, \quad a=1,2,3,
$$

\footnotetext{
${ }^{32}$ Expressions for $L^{(a)}$ and $Q^{(a a+1)}(6.58)-(6.63)$ imply that $k_{\max }=x^{(1)}+x^{(2)}+2 y^{(1)}+2 y^{(2)}+y^{(3)}$. Relations for $y^{(a)}$ 6.67)-6.69) then lead to 6.73).

${ }^{33}$ If $x^{(1)}=x^{(2)}=0$, then restrictions $(6.74)$ become the restrictions well known in the angular momentum theory: $\left|s^{(1)}-s^{(2)}\right| \leq s^{(3)} \leq s^{(1)}+s^{(2)}$.
} 
in (7.2) and ignoring contribution of oscillators carrying a subscript $n>1$. Adopting the simplified notation (7.5) for linear forms $L^{(a)} \equiv L_{1}^{(a)}, B^{(a)} \equiv B_{1}^{(a)}(7.3)$, and quadratic forms $Q^{(a b)} \equiv Q_{11}^{(a b)}$ (7.4), we see that vertex (7.2) takes the form

$$
p_{[3]}^{-}=p_{[3]}^{-}\left(L^{(a)} ; Q^{(a a+1)}, Q^{(a a)}\right) .
$$

Vertex (7.6) describes the interaction of the towers of massive totally symmetric fields (2.19). We next obtain the vertex for massive totally symmetric spin $s^{(1)}, s^{(2)}, s^{(3)}$ fields. The massive totally symmetric spin $s^{(a)}$ fields are described by the respective ket-vectors $\left|\phi_{s^{(a)}}\right\rangle$. The ket-vectors of massive fields $\left|\phi_{s^{(a)}}\right\rangle, a=1,2,3$, can be obtained from (2.14) by replacement $s \rightarrow s^{(a)}, \alpha^{I} \rightarrow \alpha^{(a) I}$, $\alpha \rightarrow \alpha^{(a)}$ in (2.14). Because $\left|\phi_{s^{(a)}}\right\rangle$ are respective degree $s^{(a)}$ homogeneous polynomials in $\alpha^{(a) I}$, $\alpha^{(a)}$ (see (2.16)), it is obvious that the vertex we are interested in must satisfy the equations

$$
\left(\alpha^{(a) I} \bar{\alpha}^{(a) I}+\alpha^{(a)} \bar{\alpha}^{(a)}-s^{(a)}\right)\left|p_{[3]}^{-}\right\rangle=0, \quad a=1,2,3,
$$

which tell us that the vertex $p_{[3]}^{-}$must be a degree $s^{(a)}$ homogeneous polynomial in the oscillators $\alpha^{(a) I}, \alpha^{(a)}$. Taking into account that the forms $L^{(a)}$ and $Q^{(a a+1)}$ are respective degree 1 and 2 homogeneous polynomials in oscillators we obtain the general solution of Eqs.(7.7) as 34

$$
p_{[3]}^{-}\left(s^{(1)}, s^{(2)}, s^{(3)} ; x^{(1)}, x^{(2)}, x^{(3)}\right)=\prod_{a=1}^{3}\left(L^{(a)}\right)^{x^{(a)}}\left(Q^{(a a+1)}\right)^{y^{(a+2)}},
$$

where integers $y^{(a)}$ are expressible in terms of $s^{(a)}$ and three integers $x^{(a)}$ labeling the freedom of our solution,

$$
y^{(a)}=\frac{1}{2}\left(\mathbf{s}+x^{(a)}-x^{(a+1)}-x^{(a+2)}\right)-s^{(a)}, \quad a=1,2,3,
$$

and $\mathbf{s}$ is given in (5.10). The maximal number of powers of $\mathbb{P}^{I}$ in $(\mathbf{7 . 8})$, denoted by $k_{\text {max }}$, is given by 35

$$
k_{\text {max }}=\frac{1}{2}\left(\mathbf{s}+\sum_{a=1}^{3} x^{(a)}\right) .
$$

Requiring the powers of the forms $L^{(a)}$ and $Q^{(a a+1)}$ in $(7.8)$ to be non-negative integers gives the restrictions

$$
\begin{array}{ll}
x^{(a)} \geq 0, & y^{(a)} \geq 0, \quad a=1,2,3 ; \\
\mathbf{s}+\sum_{a=1}^{3} x^{(a)} & \text { even integer } .
\end{array}
$$

Using relations (7.9) allows rewriting restrictions (7.11) as 36

$$
s^{(3)}-s^{(1)}-s^{(2)}+x^{(1)}+x^{(2)} \leq x^{(3)} \leq s^{(3)}-\left|s^{(1)}-s^{(2)}-x^{(1)}+x^{(2)}\right| .
$$

\footnotetext{
${ }^{34}$ We ignore the contribution of $Q^{(a a)}$-terms of $(7.6)$ to vertex (7.8). Because of the tracelessness constraint (see the first relation in (2.18) ) the contribution of these terms to the Hamiltonian $P_{[3]}^{-}(3.3)$ vanishes.

${ }^{35}$ Expressions for $L^{(a)}$ and $Q^{(a a+1)}$ (7.3), (7.4) imply that $k_{\max }=\sum_{a=1}^{3}\left(x^{(a)}+y^{(a)}\right)$. Taking $y^{(a)}$ (7.9) into account we then find (7.10).

${ }^{36}$ If $x^{(a)}=0, a=1,2,3$, then restrictions $(7.13)$ become the restrictions well known in the angular momentum theory: $\left|s^{(1)}-s^{(2)}\right| \leq s^{(3)} \leq s^{(1)}+s^{(2)}$.
} 


\section{8 so $(\mathrm{d}-4)$ light-cone formalism}

In the preceding sections we constructed parity invariant cubic interaction vertices for massive and massless higher spin fields. We studied cubic vertices for both the mixed-symmetry and totally symmetric fields. For totally symmetric fields in Minkowski space with dimension $d>6$, the antisymmetric Levi-Civita symbol does not give a contribution to cubic vertices and therefore the parity invariant vertices we obtained constitute the complete list of cubic vertices. For totally symmetric fields in $d=4,5,6$ dimensions and mixed-symmetry fields in $d \geq 6$ dimensions, the antisymmetric Levi-Civita symbol admits new invariants and we should therefore develop a method for deriving the complete lists of cubic vertices in a systematic way. In the theories of higher spin fields, it is important to know the complete lists of cubic vertices. This is related to the fact that one needs to use all interaction vertices for constructing full to all orders in coupling constant theories of higher spin fields. We also note that vertices involving the antisymmetric Levi-Civita symbol are unavoidable in supersymmetric theories. The $\mathcal{N}=4,4 d$ supersymmetric Yang-Mills theory in light-cone superspace and most supergravity theories are important examples of such theories. Another very important example of a dynamical system whose cubic vertices involve the antisymmetric Levi-Civita symbol are superstring field theories. Cubic vertices of the superstring field theories take the form $A \exp B$, where the factor $A$ involves the antisymmetric Levi-Civita symbol. To summarize, with the prospects of potentially interesting applications to supersymmetric Yang-Mills theories, supergravity, superstring theory, and supersymmetric higher spin field theories, it is desirable to develop a method for constructing cubic vertices that allows analyzing all possible cubic vertices on an equal footing. In this section we develop such a method. Because one of the characteristic features of our method is reducing the manifest transverse $s o(d-$ $2)$ symmetry to the $s o(d-4)$ symmetry we call it the $s o(d-4)$ light-cone approach 37 .

To develop the $s o(d-4)$ light-cone approach we use equations for cubic interaction vertices in the harmonic scheme (see Section 4.1). To keep the discussion from becoming unwieldy, we restrict our attention to the case of massless fields. All that is then required is to solve the equations given in (4.26), (4.27), (4.31), (4.32):

$$
\begin{aligned}
& \mathbf{J}^{I J}\left|p_{[3]}^{-}\right\rangle=0, \\
& \left(\mathbb{P}^{I} \partial_{\mathbb{P}^{I}}+\sum_{a=1}^{3} \beta_{a} \partial_{\beta_{a}}\right)\left|p_{[3]}^{-}\right\rangle=0, \\
& \partial_{\mathbb{P}^{I}} \partial_{\mathbb{P} I}\left|p_{[3]}^{-}\right\rangle=0, \\
& X^{I J} \mathcal{P}^{J}\left|p_{[3]}^{-}\right\rangle=0, \\
& \left|p_{[3]}^{-}\right\rangle \equiv p_{[3]}^{-}\left(\mathbb{P}, \beta_{a} ; \alpha\right)|0\rangle_{1}|0\rangle_{2}|0\rangle_{3},
\end{aligned}
$$

where the angular momentum $\mathbf{J}^{I J}$ is defined in (4.7), and Eqs.(8.4) are obtainable from Eqs.(4.32) by setting $\mathrm{m}_{a}=0, a=1,2,3$. To proceed, we decompose the momentum $\mathbb{P}^{I}$, which is an $s o(d-2)$

\footnotetext{
${ }^{37}$ In the preceding studies [9], reducing the manifest $s o(d-2)$ symmetry to the $s o(d-4)$ symmetry was used to formulate superfield theory of $I I A$ superstrings. In [9], the reduction was motivated by the desire to obtain an unconstrained superfield formulation. In our study, the main motivation for the reduction is the desire to obtain the most general solution to cubic vertices for arbitrary spin fields in a Poincaré invariant theory. It is worth noticing that our method is especially convenient for studying the interaction vertices of supersymmetric theories whose unconstrained superfield formulation is based on reducing the manifest $s o(d-2)$ symmetry to the $s o(d-4)$ symmetry. The application of our method to the study of $11 d$ supergravity can be found in [59].
} 
vector, as

$$
\mathbb{P}^{I} \rightarrow \mathbb{P}^{i}, \quad \mathbb{P}^{R}, \quad \mathbb{P}^{L}, \quad i=1, \ldots, d-4,
$$

where the momentum $\mathbb{P}^{i}$ is an $s o(d-4)$ vector and complex-valued momenta $\mathbb{P}^{R}, \mathbb{P}^{L}$ are defined by

$$
\mathbb{P}^{R}=\frac{1}{\sqrt{2}}\left(\mathbb{P}^{d-2}+\mathrm{i} \mathbb{P}^{d-3}\right), \quad \mathbb{P}^{L}=\frac{1}{\sqrt{2}}\left(\mathbb{P}^{d-2}-\mathrm{i} \mathbb{P}^{d-3}\right) .
$$

In what follows, in place of the momenta $\mathbb{P}^{i}, \mathbb{P}^{R}, \mathbb{P}^{L}$, we prefer to use a dimensionfull momentum $\mathbb{P}^{L}$ and dimensionless momentum variables $q^{i}, \rho$ defined by

$$
q^{i} \equiv \frac{\mathbb{P}^{i}}{\mathbb{P}^{L}}, \quad \rho \equiv \frac{\mathbb{P}^{i} \mathbb{P}^{i}+2 \mathbb{P}^{R} \mathbb{P}^{L}}{2\left(\mathbb{P}^{L}\right)^{2}}, \quad \frac{\mathbb{P}^{R}}{\mathbb{P}^{L}}=\rho-\frac{q^{2}}{2},
$$

where $q^{2} \equiv q^{i} q^{i}$. In terms of the new momenta, vertex (8.5) takes form

$$
p_{[3]}^{-}=\left(\mathbb{P}^{L}\right)^{k} V\left(q, \rho, \beta_{a} ; \alpha\right),
$$

which implies that the vertex $p_{[3]}^{-}$is a degree $k$ monomial in $\mathbb{P}^{L}$. In terms of momenta $(8.8)$, various components of the orbital momentum (4.8) take the form

$$
\begin{aligned}
& \mathbf{L}^{R L}=q^{i} \partial_{q^{i}}+2 \rho \partial_{\rho}-\mathbb{P}^{L} \partial_{\mathbb{P} L}, \\
& \mathbf{L}^{i j}=q^{i} \partial_{q^{j}}-q^{j} \partial_{q^{i}}, \\
& \mathbf{L}^{L i}=\partial_{q^{i}}, \\
& \mathbf{L}^{R i}=\left(\rho-\frac{q^{2}}{2}\right) \partial_{q^{i}}+q^{i}\left(q^{j} \partial_{q^{j}}+2 \rho \partial_{\rho}-\mathbb{P}^{L} \partial_{\mathbb{P}^{L}}\right) .
\end{aligned}
$$

To demonstrate the main idea of introducing the variable $q^{i}$ we focus on $L i$-part of Eqs.8.1). Plugging vertex $p_{[3]}^{-}(8.9)$ and $\mathbf{L}^{L i}(8.12)$ in the $L i$-part of Eqs.(8.1), we obtain the equation

$$
\left(\partial_{q^{i}}+\mathbf{M}^{L i}\right) V\left(q, \rho, \beta_{a} ; \alpha\right)=0 \text {. }
$$

The solution of Eq.(8.14) is easily found to be

$$
V\left(q, \rho, \beta_{a} ; \alpha\right)=\widehat{E}_{q} \widetilde{V}\left(\rho, \beta_{a} ; \alpha\right), \quad \widehat{E}_{q} \equiv \exp \left(-q^{i} \mathbf{M}^{L i}\right) .
$$

Collecting the above expressions, we obtain the following representation for the vertex $p_{[3]}^{-}$:

$$
p_{[3]}^{-}=\left(\mathbb{P}^{L}\right)^{k} \widehat{E}_{q} \widetilde{V}\left(\rho, \beta_{a} ; \alpha\right),
$$

and note that in terms of the vertex $\widetilde{V}$ the $\beta$-homogeneity equation (8.2) becomes

$$
\left(\sum_{a=1}^{3} \beta_{a} \partial_{\beta_{a}}+k\right) \widetilde{V}\left(\rho, \beta_{a} ; \alpha\right)=0 .
$$

Next step is to find the dependence on the momentum $\rho$. For this, we use the $R L, R i$ and $i j$ parts of Eqs.8.1):

$$
\mathbf{J}^{R L} p_{[3]}^{-}=0, \quad \mathbf{J}^{R i} p_{[3]}^{-}=0, \quad \mathbf{J}^{i j} p_{[3]}^{-}=0 .
$$




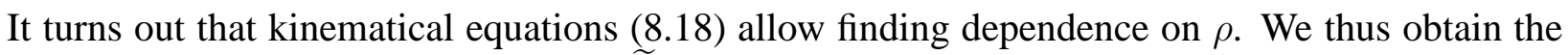
following representation for the vertex $\widetilde{V}$ (see Appendix E):

$$
\begin{aligned}
& \widetilde{V}\left(\rho, \beta_{a} ; \alpha\right)=\widehat{E}_{\rho} \widetilde{V}_{0}\left(\beta_{a} ; \alpha\right), \\
& \widehat{E}_{\rho} \equiv \sum_{n=0}^{k}(-\rho)^{n} \frac{\Gamma\left(\frac{d-4}{2}+k-n\right)}{2^{n} n ! \Gamma\left(\frac{d-4}{2}+k\right)}\left(\mathbf{M}^{L i} \mathbf{M}^{L i}\right)^{n} .
\end{aligned}
$$

In addition, the kinematical equations (8.18) lead to equations for the new vertex $\widetilde{V}_{0}\left(\beta_{a} ; \alpha\right)(8.19)$,

$$
\begin{aligned}
& \left(\mathbf{M}^{R L}-k\right) \widetilde{V}_{0}\left(\beta_{a} ; \alpha\right)=0, \\
& \mathbf{M}^{R i} \widetilde{V}_{0}\left(\beta_{a} ; \alpha\right)=0, \\
& \mathbf{M}^{i j} \widetilde{V}_{0}\left(\beta_{a} ; \alpha\right)=0
\end{aligned}
$$

while the $\beta$-homogeneity equation (8.17) takes the form

$$
\left(\sum_{a=1}^{3} \beta_{a} \partial_{\beta_{a}}+k\right) \widetilde{V}_{0}\left(\beta_{a} ; \alpha\right)=0 .
$$

The dependence on the transverse space momentum $\mathbb{P}^{I}$ is thus found explicitly and we obtain the following representation for the cubic interaction vertex:

$$
p_{[3]}^{-}\left(\mathbb{P}, \beta_{a} ; \alpha\right)=\left(\mathbb{P}^{L}\right)^{k} \widehat{E}_{q} \widehat{E}_{\rho} \widetilde{V}_{0}\left(\beta_{a} ; \alpha\right)
$$

where $\widetilde{V}_{0}$ satisfies Eqs. 8.21)-8.24). One can make sure that vertex (8.25) satisfies the harmonic equation (8.3) (see Appendix E). We now proceed to the last step of our method.

The last step is to find the dependence of the vertex $\widetilde{V}_{0}\left(\beta_{a} ; \alpha\right)$ on the three light-cone momenta, $\beta_{1}, \beta_{2}, \beta_{3}$. Finding the dependence of $\widetilde{V}_{0}\left(\beta_{a} ; \alpha\right)$ on the momenta $\beta_{a}$ is the most difficult point in the framework of the light-cone approach because the vertices $p_{[3]}^{-}$and $\widetilde{V}_{0}\left(\beta_{a} ; \alpha\right)$ are not polynomials in the light-cone momenta $\beta_{a}$ in general, i.e. there is no locality condition with respect to the light-cone coordinate $x^{-}$. But our approach, which is algebraic in nature, allows finding simple representation of the dependence on $\beta_{a}$. We proceed as follows. Because of the second relation in (4.1), the vertex $\widetilde{V}_{0}$ depends on two light-cone momenta. Therefore, we need two equations to find $\widetilde{V}_{0}$. One of equations is given in (8.24). Our basic observation is that the second equation for $\widetilde{V}_{0}$ can be obtained from locality equations (8.4). It is easy to understand if the $s o(d-2)$ invariance equations (8.1) are satisfied then in order to respect all locality equations (8.4) it is sufficient to solve the $L$-part of locality equations $(8.4)$, which in the $s o(d-4)$ notation takes the form

$$
\left(X^{L R} \mathcal{P}^{L}+X^{L i} \mathcal{P}^{i}\right)\left|p_{[3]}^{-}\right\rangle=0 \text {. }
$$

Using the representation for $p_{[3]}^{-}$given in (8.25), we can prove that locality equation (8.26) amounts to requirement that the vertex $\widetilde{V}_{0}$ satisfies the equation (see Appendix E)

$$
\sum_{a=1}^{3} \check{\beta}_{a}\left(\beta_{a} \partial_{\beta_{a}}+M^{(a) R L}\right) \widetilde{V}_{0}\left(\beta_{a} ; \alpha\right)=0 .
$$


We note that the consistence requirement for Eqs.(8.22), (8.27) leads to the equation

$$
\sum_{a=1}^{3} \check{\beta}_{a} M^{(a) R i} \widetilde{V}_{0}\left(\beta_{a} ; \alpha\right)=0 .
$$

It is easy to see that Eqs. (8.21), (8.24), (8.27) allow finding the dependence of $\widetilde{V}_{0}$ on the light-cone momenta $\beta_{a}$ completely:

$$
\widetilde{V}_{0}\left(\beta_{a} ; \alpha\right)=\widehat{E}_{\beta} \bar{V}_{0}(\alpha), \quad \widehat{E}_{\beta} \equiv \prod_{a=1}^{3} \beta_{a}^{-M^{(a) R L}} .
$$

Using (8.29), it can be shown that Eqs.(8.22), (8.28) amount to the following equations for $\bar{V}_{0}(\alpha)$ :

$$
M^{(1) R i} \bar{V}_{0}(\alpha)=M^{(2) R i} \bar{V}_{0}(\alpha)=M^{(3) R i} \bar{V}_{0}(\alpha),
$$

while Eqs.8.21), (8.23) lead to the equations

$$
\begin{aligned}
& \left(\mathbf{M}^{R L}-k\right) \bar{V}_{0}(\alpha)=0, \\
& \mathbf{M}^{i j} \bar{V}_{0}(\alpha)=0 .
\end{aligned}
$$

Collecting all the steps above, we obtain the following representation for the cubic vertex:

$$
p_{[3]}^{-}\left(\mathbb{P}, \beta_{a} ; \alpha\right)=\left(\mathbb{P}^{L}\right)^{k} \widehat{E}_{q} \widehat{E}_{\rho} \widehat{E}_{\beta} \bar{V}_{0}(\alpha),
$$

where the vertex $\bar{V}_{0}(\alpha)$ depends only on the spin degrees of freedom, denoted by $\alpha$, and satisfies Eqs. (8.30)-(8.32). The dependence on the transverse space momentum $\mathbb{P}^{I}$ and the light-cone momenta $\beta_{a}$ is thus fixed explicitly. An attractive feature of the representation (8.33) for the vertex is that it is valid for an arbitrary realization of spin degrees of freedom. Because we used the general form of the angular momentum $\mathbf{J}^{I J}$ in deriving (8.33), the solution for $p_{[3]}^{-}(8.33)$ is universal and is valid for an arbitrary Poincaré invariant theory. Various theories differ by: (i) the spin operators $M^{I J}$; (ii) the vertex $\bar{V}_{0}(\alpha)$ that depends only on spin variables $\alpha$.

We now demonstrate that the remaining Eqs. (8.30)-(8.32) can be recast into a form that admits a purely group theoretical interpretation. For this, we use the well-known fact that each irrep of the $s o(d-2)$ algebra can be realized as an induced representation by inducing from the $s o(d-4) \otimes s o(2)$ subalgebra. Let $M^{I J}$ be the $s o(d-2)$ algebra generators realized in the $s o(d-2)$ algebra irreps labeled by Gelfand-Zetlin labels $s_{1}, s_{2}, \ldots, s_{\nu}$. Then the generators $M^{I J}$ obtained via the method of induced representations take the form

$$
\begin{aligned}
& M^{R i}=\bar{\zeta}^{i}, \\
& M^{R L}=-\zeta \bar{\zeta}+s_{1}, \\
& M^{i j}=\zeta^{i} \bar{\zeta}^{j}-\zeta^{j} \bar{\zeta}^{i}+S^{i j}, \\
& M^{L i}=-\frac{1}{2} \zeta^{2} \bar{\zeta}^{i}+\zeta^{i} \zeta \bar{\zeta}+S^{i j} \zeta^{j}-s_{1} \zeta^{i},
\end{aligned}
$$

where $\zeta \bar{\zeta} \equiv \zeta^{i} \bar{\zeta}^{i}, \zeta^{2} \equiv \zeta^{i} \zeta^{i}$ and $S^{i j}$ stands for the $s o(d-4)$ algebra generators. The generators $S^{i j}$ are realized in the $s o(d-4)$ algebra irreps labeled by the Gelfand-Zetlin labels $s_{2}, \ldots, s_{\nu}$. The oscillators $\zeta^{i}, \bar{\zeta}^{i}$, being vectors of the $s o(d-4)$ algebra, satisfy the commutator $\left[\bar{\zeta}^{i}, \zeta^{j}\right]=\delta^{i j}[38$.

\footnotetext{
${ }^{38}$ Alternative convenient realization of $\zeta^{i}$ and $\bar{\zeta}^{i}$ is to treat $\zeta^{i}$ as complex-valued vector and $\bar{\zeta}^{i}$ as derivative in $\zeta^{i}$ : $\bar{\zeta}^{i} \equiv \partial_{\zeta^{i}}$
} 
To apply the spin operators $M^{I J}$ (8.34)-(8.37) to the analysis of cubic vertices, we attach external line index to all quantities given in (8.34)-(8.37), i.e. we introduce $M^{(a) I J}, \zeta^{(a) i}, S^{(a) i j}$, $s_{1}^{(a)}, a=1,2,3$. Using the representation for the spin operators $M^{(a) R i}$ (8.34) we then obtain the solution of Eqs. (8.30)

$$
\bar{V}_{0}(\alpha)=G\left(\boldsymbol{\zeta}, \alpha_{S}\right), \quad \boldsymbol{\zeta}^{i} \equiv \sum_{a=1}^{3} \zeta^{(a) i},
$$

where $\alpha_{S}$ stands for spin variables related to the $s o(d-4)$ symmetries. Plugging $\bar{V}_{0}(\alpha)(8.38)$ into Eqs.8.31), (8.32) we find the equations for the vertex $G$,

$$
\begin{aligned}
& \left(\mathbf{L}^{i j}(\boldsymbol{\zeta})+\mathbf{S}^{i j}\right) G=0, \quad \mathbf{S}^{i j} \equiv \sum_{a=1}^{3} S^{(a) i j}, \\
& \left(\boldsymbol{\zeta}^{i} \partial_{\boldsymbol{\zeta}^{i}}+k-\sum_{a=1}^{3} s_{1}^{(a)}\right) G=0,
\end{aligned}
$$

where $\mathbf{L}^{i j}(\boldsymbol{\zeta})$ is defined similarly to (4.8). Equations (8.39) for the vertex $G$ are purely group theoretical equations. In the group theoretical language, $G$ is the generating function of Clebsch -Gordan coefficients connecting one vector representation $\zeta^{i}$ and three representations whose spin matrices are given by $S^{(a) i j}, a=1,2,3$. Thus, the final expression for the vertex $p_{[3]}^{-}$is given by

$$
p_{[3]}^{-}=\left(\mathbb{P}^{L}\right)^{k} \widehat{E}_{\rho} V_{0}, \quad V_{0} \equiv \widehat{E}_{q} \widehat{E}_{\beta} G\left(\boldsymbol{\zeta}, \alpha_{S}\right) .
$$

In this formula, the operators $\widehat{E}_{q}, \widehat{E}_{\rho}, \widehat{E}_{\beta}$ are given in (8.15), (8.20), (8.29), while the spin operators $M^{I J}$ are given in (8.34)-(8.37). The vertex $G$ is fixed by Eqs.(8.39), (8.40). We next demonstrate how the general solution 8.41 can be used in concrete applications.

\subsection{Cubic interaction vertices for massless totally symmetric fields in $5 d$ Minkowski space}

$5 d$ flat space is the simplest case where the advantages of the $s o(d-4)$ light-cone approach can be demonstrated. In $5 d$ flat space all physical massless fields are classified by irreps of the $s o(3)$ algebra. Since irreps of the $s o(3)$ algebra are labeled by one label, all massless fields in $5 d$ flat space can be described by totally symmetric tensors fields of the $s o(3)$ algebra; we therefore restrict our attention to the study of cubic interaction vertices for the totally symmetric fields.

For the $5 d$ space, the indices $i, j$ that label $d-4$ directions take one value: $i, j=1$. To simplify our expressions, we use the short notation for the dimensionless momentum $q^{i}(8.8)$ and the spin variable $\zeta^{i}$ :

$$
q \equiv q^{1}, \quad \zeta \equiv \zeta^{1} .
$$

The spin operators $M^{I J}$ (8.34)-8.37) then take the form

$$
M^{R L}=-\zeta \bar{\zeta}+s, \quad M^{R 1}=\bar{\zeta}, \quad M^{L 1}=\frac{1}{2} \zeta^{2} \bar{\zeta}-s \zeta,
$$

where $s \equiv s_{1}$. In considering cubic vertices, the quantities $M^{I J}, \zeta$, and $s$ should be equipped with an external line index to become $M^{(a) I J}, \zeta^{(a)}$, and $s^{(a)}, a=1,2,3$. With this convention, the solution of Eq. (8.40) takes the form

$$
G=\boldsymbol{\zeta}^{\mathbf{s}-k}, \quad \boldsymbol{\zeta} \equiv \sum_{a=1}^{3} \zeta^{(a)}, \quad \mathbf{s} \equiv \sum_{a=1}^{3} s^{(a)} .
$$


Using relations for action of the spin operators $M^{(a) R L}, M^{(a) L 1}$ :

$$
\begin{aligned}
& \prod_{a=1}^{3} \beta_{a}^{-M^{(a) R L}} f(\boldsymbol{\zeta})=\prod_{a=1}^{3} \beta_{a}^{-s^{(a)}} f(\boldsymbol{\beta} \cdot \boldsymbol{\zeta}), \quad \boldsymbol{\beta} \cdot \boldsymbol{\zeta} \equiv \sum_{a=1}^{3} \beta_{a} \zeta^{(a)} \\
& e^{-q\left(\zeta^{2} \bar{\zeta}+b \zeta\right)} f(\zeta)=(1+q \zeta)^{-b} f\left(\frac{\zeta}{1+q \zeta}\right),
\end{aligned}
$$

we obtain the following representation for vertex $V_{0}(8.41)$ :

$$
V_{0}\left(s^{(1)}, s^{(2)}, s^{(3)} ; k\right)=\mathcal{Z}^{\mathbf{s}-k} \prod_{a=1}^{3}\left(\mathcal{B}^{(a)}\right)^{s^{(a)}}
$$

where we use the notation

$$
\mathcal{B}^{(a)} \equiv \frac{1}{\beta_{a}}\left(1+\frac{1}{2} q \zeta^{(a)}\right)^{2}, \quad \mathcal{Z} \equiv \sum_{a=1}^{3} \frac{\beta_{a} \zeta^{(a)}}{1+\frac{1}{2} q \zeta^{(a)}}
$$

For vertex $V_{0}(8.47)$ to be sensible it should be polynomial with respect to the spin variables $\zeta^{(a)}$. We therefore impose the restrictions on spin values $s^{(a)}$ and the number of derivatives $k$ :

$$
\mathbf{s}-k \geq 0, \quad 2 s^{(a)} \geq \mathbf{s}-k, \quad a=1,2,3,
$$

which coincide with those in (5.11). We rewrite restrictions (8.49) as

$$
\mathbf{s}-2 s_{\min } \leq k \leq \mathbf{s}, \quad s_{\min } \equiv \min _{a=1,2,3} s^{(a)} .
$$

Comparing with restrictions (5.11), (5.12), we see that the number $\mathrm{s}-k$ is not restricted to be even integer in the case under consideration. This is, for fixed spin values $s^{(1)}, s^{(2)}, s^{(3)}$, the integer $k$ takes the values (see (8.50) $)$

$$
k=\mathbf{s}, \mathbf{s}-1, \mathbf{s}-2, \ldots, \mathbf{s}-2 s_{\min } .
$$

This implies that for fixed spin values $s^{(1)}, s^{(2)}, s^{(3)}$, the number of allowed vertices $p_{[3]}^{-}\left(s^{(1)}, s^{(2)}, s^{(3)} ; k\right)$ that can be constructed is given by

$$
\mathrm{N}\left(s^{(1)}, s^{(2)}, s^{(3)}\right)=2 s_{\min }+1 .
$$

Comparing (8.52) with (5.16), we conclude that the $s o(d-4)$ formalism gives additional $s_{\text {min }}$ vertices compared with those obtained in the $s o(d-2)$ formalism of Section 5.1 without using the antisymmetric Levi-Civita symbol. It is these additional $s_{\min }$ vertices that could be built using the antisymmetric Levi-Civita symbol $\epsilon^{I J K}$ in the $s o(d-2)(s o(3)$ for $d=5)$ formalism 39 .

To summarize, the complete list of cubic vertices for the massless spin $s^{(1)}, s^{(2)}, s^{(3)}$ fields in $5 d$ space involves $s_{\text {min }}+1$ parity invariant vertices $p_{[3]}^{-}\left(s^{(1)}, s^{(2)}, s^{(3)} ; k\right)$ with the number of derivatives given by

$$
k=\mathbf{s}, \mathbf{s}-2, \ldots, \mathbf{s}-2 s_{\min }, \quad \text { for parity invariant vertices, }
$$

\footnotetext{
39 We recall that vertices not involving the antisymmetric Levi-Civita symbol are referred to as parity invariant vertices, while vertices involving the antisymmetric Levi-Civita symbol are referred to as parity violating vertices.
} 
and $s_{\text {min }}$ parity violating vertices $p_{[3]}^{-}\left(s^{(1)}, s^{(2)}, s^{(3)} ; k\right)$ with the number of derivatives given by

$$
k=\mathbf{s}-1, \mathbf{s}-3, \ldots, \mathbf{s}-2 s_{\min }+1, \quad \text { for parity violating vertices } .
$$

A remarkable property of the $s o(d-4)$ formalism is that it allows constructing both the parity invariant and parity violating vertices on an equal footing. This is especially important in applications to supersymmetric theories that involve vertices of both these types. In Table III we present those parity violating cubic vertices whose Lorentz covariant counterparts are available in the literature 40 . The parity invariant cubic vertices for arbitrary $d \geq 4$ were given in Tables I, II.

Table III. Parity violating cubic interaction vertices for massless totally symmetric fields in $5 d$ space. In the 3rd column, $A \mu$ stands for the Abelian spin 1 field, the matrices $R_{\mu \nu}$ and $\omega_{\mu}$ stand for the Riemann tensor $R_{\mu \nu}^{A B}$ and Lorentz connection $\omega_{\mu}^{A B}$, and $\operatorname{Tr}$ denotes trace over Lorentz indices $A, B$.

\begin{tabular}{|c|c|c|}
\hline $\begin{array}{c}\text { Spin values and } \\
\text { number of derivatives } \\
s^{(1)}, s^{(2)}, s^{(3)} ; k\end{array}$ & $\begin{array}{c}\text { Light-cone } \\
\text { vertex } \\
V_{0}\left(s^{(1)}, s^{(2)}, s^{(3)} ; k\right)\end{array}$ & $\begin{array}{c}\text { Covariant } \\
\text { Lagrangian }\end{array}$ \\
\hline $1,1,1 ; 2$ & $\mathcal{B}^{(1)} \mathcal{B}^{(2)} \mathcal{B}^{(3)} \mathcal{Z}$ & $\epsilon^{\mu \nu \rho \sigma \lambda} F_{\mu \nu} F_{\rho \sigma} A_{\lambda}$ \\
\hline $1,2,2 ; 4$ & $\mathcal{B}^{(1)}\left(\mathcal{B}^{(2)} \mathcal{B}^{(3)}\right)^{2} \mathcal{Z}$ & $\epsilon^{\mu \nu \rho \sigma \lambda} \operatorname{Tr}\left(R_{\mu \nu} R_{\rho \sigma}\right) A_{\lambda}$ \\
\hline $2,2,2 ; 3$ & $\left(\mathcal{B}^{(1)} \mathcal{B}^{(2)} \mathcal{B}^{(3)}\right)^{2} \mathcal{Z}^{3}$ & $\mathcal{L}($ see Ref. $[68])$ \\
\hline $2,2,2 ; 5$ & $\left(\mathcal{B}^{(1)} \mathcal{B}^{(2)} \mathcal{B}^{(3)}\right)^{2} \mathcal{Z}$ & $\epsilon^{\mu \nu \rho \sigma \lambda} \operatorname{Tr}\left(R_{\mu \nu} R_{\rho \sigma} \omega_{\lambda}\right)$ \\
\hline $3,3,3 ; 4$ & $\left(\mathcal{B}^{(1)} \mathcal{B}^{(2)} \mathcal{B}^{(3)}\right)^{3} \mathcal{Z}^{5}$ & $\mathcal{L}(\operatorname{see} \operatorname{Ref} .[40])$ \\
\hline
\end{tabular}

\subsection{Cubic interaction vertices for massless totally symmetric and mixed- symmetry fields in $6 d$ Minkowski space}

In $6 d$ flat space all physical massless fields are classified by irreps of the $s o(4)$ algebra. Since irreps of the $s o(4)$ algebra are labeled by two Gelfand-Zetlin labels $s_{1}, s_{2}, s_{1} \geq\left|s_{2}\right|$, massless fields in $6 d$ flat space are described by $s o(4)$ totally symmetric tensor fields $\left(s_{1} \geq 0, s_{2}=0\right)$ and $s o(4)$ mixed-symmetry tensor fields $\left(s_{2} \neq 0\right)$. For the massless mixed-symmetry fields, $6 d$ flat space is the simplest case where advantages of the $s o(d-4)$ light-cone approach can be demonstrated. Our approach allows us to build cubic vertices for all massless fields on an equal footing.

Since the indices $i, j$ labeling $d-4$ directions take two values for $d=6, i, j=1,2$, we prefer to use the complex coordinates

$$
x=\frac{1}{\sqrt{2}}\left(x^{1}+\mathrm{i} x^{2}\right), \quad \bar{x}=\frac{1}{\sqrt{2}}\left(x^{1}-\mathrm{i} x^{2}\right),
$$

in place of $d-4$ coordinates $x^{1}, x^{2}$. In the complex coordinates, the indices $i, j$ range over $x$ and $\bar{x}$ and the dimensionless momentum $q^{i}(8.8)$ and the spin variable $\zeta^{i}$ are decomposed as

$$
q^{i}=q^{x}, q^{\bar{x}} ; \quad \zeta^{i}=\zeta^{x}, \zeta^{\bar{x}}
$$

\footnotetext{
${ }^{40}$ In the literature, we have not found the parity violating covariant Lagrangian for low spin $s=1,2$ fields that corresponds to our light-cone vertex $V_{0}(1,1,2 ; 3)$. The covariant Lagrangian in the 4th line of Table III is invariant only under linearized gauge transformations.
} 
while the generator of the $s o(2)$ algebra $S^{i j}$ is expressed in terms of Gelfand-Zetlin label $s_{2}$,

$$
S^{x \bar{x}}=s_{2} .
$$

This implies the following representation for the spin operators $M^{L i}$ (8.37):

$$
M^{L \bar{x}}=\left(\zeta^{\bar{x}}\right)^{2} \bar{\zeta}^{x}-\left(s_{1}+s_{2}\right) \zeta^{\bar{x}}, \quad M^{L x}=\left(\zeta^{x}\right)^{2} \bar{\zeta}^{\bar{x}}-\left(s_{1}-s_{2}\right) \zeta^{x} .
$$

We note that the Gelfand-Zetlin labels $s_{1}$ and $s_{2}$ of the $s o(4)$ algebra irreps can be related to labels $j_{1}, j_{2}$ of irreps of $s o(3)_{1}$ and $s o(3)_{2}$ algebras that enter the decomposition $s o(4)=s o(3)_{1} \oplus s o(3)_{2}$ :

$$
j_{1}=\frac{1}{2}\left(s_{1}+s_{2}\right), \quad j_{2}=\frac{1}{2}\left(s_{1}-s_{2}\right) .
$$

To study cubic vertices the quantities $j_{1}, j_{2}, \zeta^{i}$ should be equipped with external line index $a=$ $1,2,3$, i.e. we should introduce $j_{1}^{(a)}, j_{2}^{(a)}, \zeta^{(a) i}$. With this convention, the solution of Eqs. (8.39), (8.40) takes the form

$$
G=\left(\boldsymbol{\zeta}^{\bar{x}}\right)^{\mathbf{j}_{1}-\frac{k}{2}}\left(\boldsymbol{\zeta}^{x}\right)^{\mathbf{j}_{2}-\frac{k}{2}}, \quad \boldsymbol{\zeta}^{i} \equiv \sum_{a=1}^{3} \zeta^{(a) i}, \quad \mathbf{j}_{\sigma} \equiv \sum_{a=1}^{3} j_{\sigma}^{(a)}
$$

Using spin operators (8.58) and relations $(8.45),(8.46)$, we obtain the cubic vertex

$$
V_{0}\left(j_{\sigma}^{(a)} ; k\right)=\prod_{\sigma=1,2} \mathcal{Z}_{\sigma}^{\mathbf{j}_{\sigma}-\frac{k}{2}} \prod_{\substack{a=1,2,3 \\ \sigma=1,2}}\left(\mathcal{B}_{\sigma}^{(a)}\right)^{j_{\sigma}^{(a)}}
$$

where we use the notation

$$
\begin{gathered}
\mathcal{B}_{\sigma}^{(a)} \equiv \frac{1}{\beta_{a}}\left(1+q_{\sigma} \zeta_{\sigma}^{(a)}\right)^{2}, \quad \mathcal{Z}_{\sigma} \equiv \sum_{a=1}^{3} \frac{\beta_{a} \zeta_{\sigma}^{(a)}}{1+q_{\sigma} \zeta_{\sigma}^{(a)}} \\
q_{1} \equiv q^{x}, \quad q_{2} \equiv q^{\bar{x}}, \quad \zeta_{1}^{(a)} \equiv \zeta^{(a) \bar{x}}, \quad \zeta_{2}^{(a)} \equiv \zeta^{(a) x} .
\end{gathered}
$$

For vertex $V_{0}(8.61)$ to be polynomial in the spin variables $\zeta_{\sigma}^{(a)}$, we impose the restrictions

$$
\begin{array}{ll}
2\left(\mathbf{j}_{\sigma}-2 j_{\sigma}^{(a)}\right) \leq k \leq 2 \mathbf{j}_{\sigma}, & a=1,2,3 ; \quad \sigma=1,2 ; \\
2 \mathbf{j}_{\sigma}-k \quad \text { even integers }, & \sigma=1,2 .
\end{array}
$$

Restrictions (8.64) amount to the restrictions

$$
2 \max _{\sigma=1,2}\left(\mathbf{j}_{\sigma}-2 \min _{a=1,2,3} j_{\sigma}^{(a)}\right) \leq k \leq 2 \min _{\sigma=1,2} \mathbf{j}_{\sigma}
$$

From (8.65), (8.66), we see that for fixed spin values $j_{\sigma}^{(a)}$, the number of cubic interaction vertices $p_{[3]}^{-}\left(j_{\sigma}^{(a)} ; k\right)$ that can be constructed is given by

$$
\mathbf{N}\left(j_{\sigma}^{(a)}\right)=\min _{\sigma=1,2} \mathbf{j}_{\sigma}-\max _{\sigma=1,2}\left(\mathbf{j}_{\sigma}-2 \min _{a=1,2,3} j_{\sigma}^{(a)}\right)+1 .
$$

To summarize, for fixed spin values $j_{\sigma}^{(a)}$, restrictions (8.65), (8.66) define all possible (parity invariant and parity violating) cubic vertices that can be built for the massless totally symmetric and mixed-symmetry fields in $6 d$ flat space. The number of these vertices is given by (8.67). 
We now restrict our attention to the totally symmetric fields and compare the complete list of vertices obtained using the $s o(d-4)$ method in this section and the list of parity invariant vertices obtained by the $s o(d-2)$ method in Section 5.1. All that is required is to compare the restrictions (8.65), (8.66) for the totally symmetric fields and restrictions (5.12), (5.13). To adapt restrictions (8.65), (8.66) to the totally symmetric fields we set $s_{2}^{(a)}=0, a=1,2,3$. Relations (8.59) then give $j_{1}^{(a)}=j_{2}^{(a)}=s_{1}^{(a)} / 2$. Using the identification $s_{1}^{(a)} \equiv s^{(a)}$, we see that restrictions (8.65), (8.66) for the totally symmetric fields coincide with those of the $s o(d-2)$ approach, (5.12), (5.13). This implies that for massless totally symmetric fields in $6 d$, the complete list of cubic vertices obtained using the $s o(d-4)$ method in this section coincides with the list of parity invariant cubic vertices obtained using the $s o(d-2)$ method in Section 5.1, i.e. all cubic vertices for the massless totally symmetric fields in $6 d$ are parity invariant. Thus, in contrast to the $5 d$ case the antisymmetric Levi-Civita symbol does not lead to new cubic vertices for the massless totally symmetric fields in $6 d$.

In a manifestly Lorentz covariant formulation, the massless mixed-symmetry fields in $6 d$ flat space are described by a set of the tensor fields whose $S O(5,1)$ space-time tensor indices have the structure of a Young tableaux with two rows. The study of interaction vertices for massless mixed-symmetry fields for arbitrary $d$ in the framework of covariant approach can be found in [69].

\section{Conclusions}

Using the light-cone formalism we have developed various methods for constructing cubic interaction vertices for higher spin fields propagating in flat space. We applied these methods to construct a wide class of cubic interaction vertices for massless and massive arbitrary spin fields. For mixed-symmetry fields in space of arbitrary dimension, we obtained the generating function of the parity invariant cubic vertices. We believe that this generating function involves all possible parity invariant vertices. To classify these vertices (i.e. to find restrictions on powers of derivatives in a vertex for three fields carrying various values of spins) it is necessary to single out irreducible components of the reducible sets of fields as this was done in the case of totally symmetric fields. It seems likely that the easiest way to do that is to apply the $s o(d-4)$ method to the generating solution for vertices. We hope to study this issue elsewhere. We emphasize that the generating form of the cubic vertices is very convenient for studying higher order interaction corrections in the theories of higher spin fields. We believe that the study these corrections will allow us to find the generating function explicitly.

Our results should have a number of the following interesting applications and generalizations.

i) We studied interaction vertices for bosonic fields. It would be interesting to extend the methods developed in this paper to the case of fermionic fields and apply these results to interaction vertices of the closed superstring field theory.

ii) The light-cone gauge formulation of free fields in $A d S$ space was developed in [34]-[36]. It would be interesting to extend the methods in this paper to study cubic interaction vertices for fields propagating in $A d S$ space. This should be relatively straightforward because the light-cone gauge formulation of the field dynamics in $A d S$ space provides certain simplifications.

iii) Another interesting application is related to certain massless (nonsupersymmetric) triplets in $d=11$, the dimension of M-theory. It was found in [70] that some irreps of the $s o(9)$ algebra naturally group together into triplets to be referred to as Euler triplets which are such that bosonic and fermionic degrees of freedom match up the same way as in $11 d$ supergravity (see also [71]- 
[73]). Later on it was conjectured that these triplets might be organized in a relativistic theory so that this theory would presumably be finite. The methods we developed in this paper and those we used for studying 11d supergravity [59] admit a straightforward generalization to higher spin Euler triplets. We hope to return to these problems in future publications.

Acknowledgments. We thank N. Boulanger for informative communications and A. Semikhatov for useful comments. This work was supported by the INTAS project 03-51-6346, by the RFBR Grant No.05-02-17654, RFBR Grant for Leading Scientific Schools, Grant No. LSS-4401.2006.2 and Russian Science Support Foundation.

\section{Appendix A Derivation of expressions for $\mathcal{X}^{I}($ (4.18)$)$.}

In this Appendix we derive formulas (4.17), (4.18). To this end we note the relation

$$
p_{a}^{I}=-\frac{\beta_{a} \check{\beta}_{a}}{3 \hat{\beta}} \mathbb{P}^{I}+\frac{\beta_{a}}{3} \widetilde{P}^{I}, \quad \widetilde{P}^{I} \equiv \sum_{a=1}^{3} \frac{p_{a}^{I}}{\beta_{a}} .
$$

Making use of (A.1) it is easy to derive the following helpful relations:

$$
\begin{aligned}
& \sum_{a=1}^{3} p_{a}^{I} \check{p}_{a}^{J}=\mathbb{P}^{I} \widetilde{P}^{J}-\mathbb{P}^{J} \widetilde{P}^{I}, \quad \check{p}_{a}^{I} \equiv p_{a+1}^{I}-p_{a+2}^{I}, \\
& \sum_{a=1}^{3} \check{\beta}_{a} p_{a}^{-}=-\widetilde{P}^{I} \mathbb{P}^{I}-\sum_{a=1}^{3} \frac{\check{\beta}_{a}}{2 \beta_{a}} \mathrm{~m}_{a}^{2}, \\
& \sum_{a=1}^{3} \frac{1}{\beta_{a}} M^{(a) I J} p_{a}^{J}=-\frac{1}{3 \hat{\beta}} \sum_{a=1}^{3} \check{\beta}_{a} M^{(a) I J} \mathbb{P}^{J}+\frac{1}{3} \mathbf{M}^{I J} \widetilde{P}^{J} .
\end{aligned}
$$

In Eq.4.12) the operator $\mathbf{J}^{-I \dagger}(4.13)$ is realized as differential operator with respect to the momenta $p_{a}^{I}, \beta_{a}$, which acts on the vertex $p_{[3]}^{-}\left(p_{a}, \beta_{a} ; \alpha\right)=p_{[3]}^{-}\left(\mathbb{P}, \beta_{a} ; \alpha\right)$. To derive formulas (4.17), (4.18) we should realize the operator $\mathbf{J}^{-I^{\dagger}}$ as differential operator with respect to the momenta $\mathbb{P}^{I}, \beta_{a}$, which acts on vertex $p_{[3]}^{-}\left(\mathbb{P}, \beta_{a} ; \alpha\right)$ (4.4). To this end we consider an action of different pieces of the operator $\mathbf{J}^{-I \dagger}(4.13)$ on the vertex $p_{[3]}^{-}\left(p_{a}, \beta_{a} ; \alpha\right)=p_{[3]}^{-}\left(\mathbb{P}, \beta_{a} ; \alpha\right)$ and obtain the relations

$$
\begin{aligned}
\sum_{a=1}^{3} p_{a}^{-} \partial_{p_{a}^{I}} p_{[3]}^{-}\left(p_{b}, \beta_{b} ; \alpha\right) & =\frac{1}{3} \sum_{a=1}^{3} \check{\beta}_{a} p_{a}^{-} \partial_{\mathbb{P}^{I}} p_{[3]}^{-}\left(\mathbb{P}, \beta_{b} ; \alpha\right) \\
& =\left(-\frac{1}{3} \widetilde{P}^{J} \mathbb{P}^{J}-\sum_{a=1}^{3} \frac{\check{\beta}_{a}}{6 \beta_{a}} \mathrm{~m}_{a}^{2}\right) \partial_{\mathbb{P}^{I}} p_{[3]}^{-}\left(\mathbb{P}, \beta_{b} ; \alpha\right) \\
\sum_{a=1}^{3} p_{a}^{I} \partial_{\beta_{a}} p_{[3]}^{-}\left(p_{b}, \beta_{b} ; \alpha\right) & =\sum_{a=1}^{3}\left(-\frac{1}{3 \hat{\beta}} \mathbb{P}^{I} \check{\beta}_{a} \beta_{a} \partial_{\beta_{a}}+\frac{1}{3} \widetilde{P}^{I} \beta_{a} \partial_{\beta_{a}}\right) p_{[3]}^{-}\left(p_{b}, \beta_{b} ; \alpha\right) \\
& =\frac{1}{3}\left(\mathbb{P}^{J} \widetilde{P}^{I}-\mathbb{P}^{I} \widetilde{P}^{J}\right) \partial_{\mathbb{P}^{J}} p_{[3]}^{-}\left(\mathbb{P}, \beta_{b} ; \alpha\right) \\
& +\sum_{a=1}^{3}\left(-\frac{1}{3 \hat{\beta}} \mathbb{P}^{I} \check{\beta}_{a} \beta_{a} \partial_{\beta_{a}}+\frac{1}{3} \widetilde{P}^{I} \beta_{a} \partial_{\beta_{a}}\right) p_{[3]}^{-}\left(\mathbb{P}, \beta_{b} ; \alpha\right) .
\end{aligned}
$$


In the 2nd line of (A.5) we use formula (A.3). In the first line of (A.6) we use formula (A.1), while in the 2 nd and 3rd lines of (A.6) we use the relations

$$
\sum_{a=1}^{3} \beta_{a} \partial_{\beta_{a}} \mathbb{P}^{I}=\mathbb{P}^{I}, \quad \sum_{a=1}^{3} \check{\beta}_{a} \beta_{a} \partial_{\beta_{a}} \mathbb{P}^{I}=\hat{\beta} \widetilde{P}^{I}
$$

Collecting the expressions (A.4)-(A.6) in $\mathbf{J}^{-I^{\dagger}}(4.13$ ) and taking into account Eqs.(4.5), (4.6) we note that all terms proportional to the momentum $\widetilde{P}^{I}$ are cancelled. The remaining terms then lead to the desired representation for $\mathbf{J}^{-I \dagger}\left|p_{[3]}^{-}\right\rangle$given in (4.17), (4.18).

\section{Appendix B Field redefinitions in light-cone approach}

In this appendix we discuss field redefinitions in the framework of Hamiltonian light-cone approach. Let $\phi$ be generic field. We make a field redefinition $\phi \rightarrow \widetilde{\phi}$ :

$$
\widetilde{\phi}=\phi+\sum_{n=2}^{\infty} \widetilde{\phi}_{[n]}[\phi]
$$

where $\widetilde{\phi}_{[n]}$ stands for $n$ - point contribution (having $n$ powers of the generic field $\phi$ ) to $\widetilde{\phi}$. The $\widetilde{\phi}_{[n]}$ is restricted to be local (with respect to transverse directions) functional in $\phi$. In the Hamiltonian light-cone approach we are allowed to make the field redefinitions (B.1) that satisfy the following two basic requirements: i) The field $\widetilde{\phi}$ should satisfy the light-cone canonical commutator (2.31). Field redefinitions that respect the light-cone canonical commutator (2.31) will be referred to as light-cone canonical transformations; ii) The field redefinitions (B.1) should preserve structure of the kinematical generators $(2.30)$, i.e. the field $\widetilde{\phi}$ should satisfy the equation $\widetilde{G}^{\text {kin }}=G^{k i n}[\phi]$, where $\widetilde{G} \equiv G[\widetilde{\phi}]$.

The field redefinitions (B.1) that satisfy these requirements can be introduced by using a standard procedure. Let $F[\phi]$ be generating functional of the light-cone canonical transformations. This functional has an expansion

$$
F[\phi]=\sum_{n=3}^{\infty} F_{[n]}[\phi],
$$

where $F_{[n]}[\phi]$ stands for $n$ - point contribution to $F[\phi]$ (see ( $(\underline{B .15})$ for explicit expression). We then introduce one parametric flow

$$
\partial_{s} \phi_{s}=\left[\phi_{s}, F_{s}\right], \quad F_{s} \equiv F\left[\phi_{s}\right]
$$

and note that the generic field $\phi$ and the canonically transformed field $\widetilde{\phi}(\mathbb{B} .1)$ are given by

$$
\phi \equiv \phi_{s=0}, \quad \widetilde{\phi} \equiv \phi_{s=1} .
$$

It is easy to check that the field $\widetilde{\phi}(\mathrm{B} .4)$ satisfies the light-cone canonical commutator (2.31).

We now focus on the light-cone canonical transformations that maintain the kinematical generators. Any generator $G=G[\phi]$ being canonically transformed takes the form

$$
\widetilde{G}=G_{s=1}, \quad G_{s} \equiv G\left[\phi_{s}\right],
$$


where $G_{s}$ satisfies the differential equation and the initial condition

$$
\partial_{s} G_{s}=\left[G_{s}, F_{s}\right], \quad G_{s=0}=G .
$$

Making use of (B.6) and the Taylor series expansion for $\widetilde{G}$

$$
\widetilde{G}=\left.\sum_{n=1}^{\infty} \frac{1}{n !} \partial_{s}^{n} G_{s}\right|_{s=1},
$$

we obtain the following expansion for the canonically transformed generator $\widetilde{G}(\underline{B .5})$ :

$$
\widetilde{G}=\sum_{n=1}^{\infty} \frac{1}{n !}[\ldots[G, F], \ldots F]=G+[G, F]+\frac{1}{2}[[G, F], F]+\ldots .
$$

Then using the expansion for $F[\phi](\underline{B .2})$ and expansions for the generic generator $G$ and the canonically transformed generator $\widetilde{G}$,

$$
G=\sum_{n=2}^{\infty} G_{[n]}, \quad \widetilde{G}=\sum_{n=2}^{\infty} \widetilde{G}_{[n]},
$$

we obtain the following relations for the leading terms:

$$
\begin{aligned}
& \widetilde{G}_{[2]}=G_{[2]}, \\
& \widetilde{G}_{[3]}=G_{[3]}+\left[G_{[2]}, F_{[3]}\right] \\
& \widetilde{G}_{[4]}=G_{[4]}+\left[G_{[2]}, F_{[4]}\right]+\left[G_{[3]}, F_{[3]}\right]+\frac{1}{2}\left[\left[G_{[2]}, F_{[3]}\right], F_{[3]}\right] .
\end{aligned}
$$

Now we are ready to find the light-cone canonical transformations that maintain the kinematical generators. Since the generic kinematical generators $G^{k i n}=G_{[2]}^{k i n}$ are quadratic in the field $\phi$, all that is required is to find generating function $F[\phi]$ that satisfies the equation:

$$
\widetilde{G}_{[2]}^{k i n}=G_{[2]}^{k i n}, \quad \widetilde{G}_{[n]}=0, \quad \text { for all } n \geq 3 .
$$

It is clear that suitable generating functional $F=F[\phi]$ should satisfy the equations:

$$
\left[G_{[2]}^{k i n}, F\right]=0 .
$$

By using the representation for the $n$ - point contribution to $F[\phi]$ (see expansion (

$$
F_{[n]}=\int d \Gamma_{n}\left\langle\Phi_{[n]} \mid f_{[n]}\right\rangle, \quad n \geq 3,
$$

and procedure of Section 2 we find from $(\underline{\mathrm{B} .14})$ that the densities $f_{[n]}$ are functions of $\mathbb{P}_{a b}^{I}, \beta_{a}, \alpha$,

$$
f_{[n]}=f_{[n]}\left(\mathbb{P}_{a b}, \beta_{a} ; \alpha\right),
$$

(where $\alpha$ stands for spin D.o.F) and satisfy the equations

$$
\begin{aligned}
& \left(\sum_{\{a b\}} \mathbb{P}_{a b}^{I} \partial_{\mathbb{P}_{a b}^{J}}-\mathbb{P}_{a b}^{J} \partial_{\mathbb{P}_{a b}^{I}}+\sum_{a=1}^{n} M^{(a) I J}\right)\left|f_{[n]}\right\rangle=0, \\
& \left(\sum_{\{a b\}} \mathbb{P}_{a b}^{I} \partial_{\mathbb{P}_{a b}^{I}}+\sum_{a=1}^{n} \beta_{a} \partial_{\beta_{a}}\right)\left|f_{[n]}\right\rangle=\left|f_{[n]}\right\rangle .
\end{aligned}
$$


We finish with the discussion of dependence of the cubic Hamiltonian $P_{[3]}^{-}$on the field redefinitions. From (B.11), we see that field redefinitions at cubic order are entirely governed by density $f_{[3]}$. The density $f_{[3]}=f_{[3]}\left(\mathbb{P}, \beta_{a} ; \alpha\right)(\operatorname{see}(\underline{B} .16)$ and (4.2) $)$ satisfies equations ( $\operatorname{see}(\underline{\mathrm{B} .17})$, (B.18) for $n=3$ )

$$
\mathbf{J}^{I J}\left|f_{[3]}\right\rangle=0, \quad\left(\mathbb{P}^{I} \partial_{\mathbb{P}^{I}}+\sum_{a=1}^{3} \beta_{a} \partial_{\beta_{a}}\right)\left|f_{[3]}\right\rangle=\left|f_{[3]}\right\rangle,
$$

where the angular momentum $\mathbf{J}^{I J}$ is given in (4.7). Adopting relation (B.11) for the case of $G=P^{-}$and making use of formulas (3.3), (B.15) for $n=3$, it is seen that the cubic interaction vertex $p_{[3]}^{-}$, being subjected to the field redefinitions, takes the form

$$
\widetilde{p}_{[3]}^{-}=p_{[3]}^{-}-\mathbf{P}^{-} f_{[3]},
$$

where $\mathbf{P}^{-}$is given in (4.16). From ( terms, $q=1,2, \ldots$, in the density $\widetilde{p}_{[3]}^{-}$are scheme dependent and can therefore be removed (or created) by appropriate choice of $f_{[3]}$.

\section{Appendix C Derivation of the locality equations (4.32)}

We start with the operator $\mathcal{X}^{I}$ given in (4.18)-(4.21) and note that $\mathcal{X}^{I}$ (4.18) can be decomposed into non-harmonic and harmonic parts

$$
\begin{aligned}
& \mathcal{X}^{I}=\mathcal{X}_{\text {non-harm }}^{I}+\mathcal{X}_{\text {harm }}^{I}, \\
& \mathcal{X}_{\text {non-harm }}^{I} \equiv 2 \hat{\beta} \mathbf{P}^{-} \frac{1}{2 \widehat{k}+N} X^{I J} \partial_{\mathbb{P}^{J}}, \\
& \mathcal{X}_{\text {harm }}^{I} \equiv X^{I J} \mathcal{P}^{J}+X^{I}+\left(X \delta^{I J}+\frac{\hat{\beta}}{2 \widehat{k}+N} \sum_{a=1}^{3} \frac{\mathrm{m}_{a}^{2}}{\beta_{a}} X^{I J}\right) \partial_{\mathbb{P}^{J}},
\end{aligned}
$$

where $\mathbf{P}^{-}$is given in (4.16). We note that the action of $\mathcal{X}_{\text {harm }}^{I}$ (C.3) on harmonic polynomial in $\mathbb{P}^{I}$ gives a harmonic polynomial. This is easily seen from the relations

$$
\Delta \mathcal{P}^{I}=\left(\mathbb{P}^{I}-\mathbb{P}^{J} \mathbb{P}^{J} \frac{1}{2 \widehat{k}+N+4} \partial_{\mathbb{P} I}\right) \Delta, \quad \Delta \partial_{\mathbb{P} I}=\partial_{\mathbb{P} I} \Delta, \quad \Delta \equiv \partial_{\mathbb{P} I} \partial_{\mathbb{P} I}
$$

Using (C.1)-C.3), we obtain

$$
\mathcal{X}^{I}\left|p_{[3]}^{-}\right\rangle=2 \hat{\beta} \mathbf{P}^{-} \frac{1}{2 \widehat{k}+N} X^{I J} \partial_{\mathbb{P}^{J}}\left|p_{[3]}^{-}\right\rangle+\mathcal{X}_{\text {harm }}^{I}\left|p_{[3]}^{-}\right\rangle .
$$

Since the vertex $\left|p_{[3]}^{-}\right\rangle$is chosen to be a harmonic polynomial in $\mathbb{P}^{I}$, the expression $\mathcal{X}_{\text {harm }}^{I}\left|p_{[3]}^{-}\right\rangle$in (C.5) is also a harmonic polynomial in $\mathbb{P}^{I}$. But harmonic polynomial in $\mathbb{P}^{I}$ cannot be represented as $\mathbf{P}^{-} V$, where $V$ is a polynomial in $\mathbb{P}^{I}$. This implies that in order to respect the light-cone locality condition (4.28), we should impose the equations $\mathcal{X}_{\text {harm }}^{I}\left|p_{[3]}^{-}\right\rangle=0$, which are nothing but the locality equations (4.32). The locality equations and relations (C.5), (4.23) imply the representation for $\left|j_{[3]}^{-I}\right\rangle$ given in (4.34). 


\section{Appendix D Derivation of cubic interaction vertices}

Derivation of vertex (5.2). Equations to be solved are obtainable from Eqs.(4.48), (4.46) by setting $\mathrm{m}_{1}=\mathrm{m}_{2}=\mathrm{m}_{3}=0$ in (4.46). Since the ket-vectors of massless fields (2.12) are independent of the scalar oscillators $\alpha_{n}$, the scalar oscillators $\alpha_{n}^{(a)}$ in (4.53) do not contribute to the Hamiltonian (3.3) and therefore we ignore dependence on $\alpha_{n}^{(a)}$-terms in 4.53$)$. Thus, we start with the vertex and equations

$$
\begin{aligned}
& p_{[3]}^{-}=p_{[3]}^{-}\left(B_{n}^{(a)} ; \alpha_{m n}^{(a a+1)}, \alpha_{m n}^{(a a)}\right), \\
& \sum_{m=1}^{\nu}\left(B_{m}^{(a+1)} \partial_{\alpha_{n m}^{(a a+1)}}-B_{m}^{(a+2)} \partial_{\alpha_{m n}^{(a+2 a)}}\right) p_{[3]}^{-}=0 .
\end{aligned}
$$

To analyze Eqs.(D.2) it is convenient to introduce new variables defined by

$$
x_{m n}^{(a a+1)} \equiv \frac{\alpha_{m n}^{(a a+1)}}{B_{m}^{(a)} B_{n}^{(a+1)}} .
$$

In terms of these new variables, vertex $p_{[3]}^{-}(\overline{\mathrm{D} .1})$ and Eqs.(D.2) take the form

$$
\begin{aligned}
& p_{[3]}^{-}=p_{[3]}^{-}\left(B_{n}^{(a)} ; x_{m n}^{(12)}, x_{m n}^{(23)}, x_{m n}^{(31)} ; \alpha_{m n}^{(a a)}\right) \\
& \sum_{m=1}^{\nu}\left(\partial_{x_{n m}^{(12)}}-\partial_{x_{m n}^{(31)}}\right) p_{[3]}^{-}=0 \\
& \sum_{m=1}^{\nu}\left(\partial_{x_{n m}^{(23)}}-\partial_{x_{m n}^{(122}}\right) p_{[3]}^{-}=0 \\
& \sum_{m=1}^{\nu}\left(\partial_{x_{n m}^{(31)}}-\partial_{x_{m n}^{(23)}}\right) p_{[3]}^{-}=0
\end{aligned}
$$

We solve Eqs. (D.5)-(D.7) in turn.

i) To find general solution of Eq.(D.5) we consider the appropriate characteristic equations

$$
d x_{n 1}^{(12)}=\ldots=d x_{n \nu}^{(12)}=-d x_{1 n}^{(31)}=\ldots=-d x_{\nu n}^{(31)} .
$$

Integrals of these equations are given by

$$
\tilde{y}_{n n^{\prime}}^{(12)} \equiv x_{n n^{\prime}}^{(12)}-x_{n 1}^{(12)}, \quad n^{\prime}=2, \ldots, \nu ; \quad \tilde{y}_{m n}^{(31)} \equiv x_{m n}^{(31)}+x_{n 1}^{(12)},
$$

and this implies that the general solution of Eqs.(D.5) takes the form

$$
p_{[3]}^{-}=p_{[3]}^{-}\left(B_{n}^{(a)} ; \tilde{y}_{m n^{\prime}}^{(12)}, x_{m n}^{(23)}, \tilde{y}_{m n}^{(31)} ; \alpha_{m n}^{(a a)}\right) .
$$

ii) Now we are going to find restrictions imposed by Eqs.(D.7) on the general solution (D.10). To this end we rewrite Eqs.(D.7) in terms of vertex $p_{[3]}^{-}(\underline{D .10})$ :

$$
\sum_{m=1}^{\nu}\left(\partial_{\tilde{y}_{n m}^{(31)}}-\partial_{x_{m n}^{(23)}}\right) p_{[3]}^{-}=0 .
$$

\footnotetext{
${ }^{41}$ Derivatives with respect to the scalar oscillators $\alpha_{n}^{(a)}$ vanish in (4.48), (4.46) when $\mathrm{m}_{a} \rightarrow 0$, i.e. ignoring dependence on $\alpha_{n}^{(a)}$ in the cubic interaction vertex is a self-consistent procedure in solving Eq. (4.48).
} 
As before, we solve Eqs. (D.11) by using the method of characteristic equations and we find

$$
p_{[3]}^{-}=p_{[3]}^{-}\left(B_{n}^{(a)} ; \tilde{y}_{m n^{\prime}}^{(12)}, \tilde{y}_{n^{\prime} n}^{(23)}, y_{m n}^{(31)} ; \alpha_{m n}^{(a a)}\right),
$$

where new variables $\tilde{y}_{n^{\prime} n}^{(23)}$ and $y_{m n}^{(31)}$ are defined by

$$
\tilde{y}_{n^{\prime} n}^{(23)} \equiv x_{n^{\prime} n}^{(23)}-x_{1 n}^{(23)}, \quad y_{m n}^{(31)} \equiv \tilde{y}_{m n}^{(31)}+x_{1 m}^{(23)} .
$$

iii) To solve the remaining Eqs.(D.6) we should rewrite these equations in terms of vertex $p_{[3]}^{-}$ (D.12). To this end by using the relations (see (D.9), (D.13)

$$
\tilde{y}_{m n^{\prime}}^{(12)} \equiv x_{m n^{\prime}}^{(12)}-x_{m 1}^{(12)}, \quad \tilde{y}_{n^{\prime} n}^{(23)}=x_{n^{\prime} n}^{(23)}-x_{1 n}^{(23)}, \quad y_{m n}^{(31)}=x_{m n}^{(31)}+x_{n 1}^{(12)}+x_{1 m}^{(23)},
$$

we prove that Eqs. (‥6) amount to the following equations for vertex $p_{[3]}^{-}(\overline{\mathrm{D} .12})$ :

$$
\sum_{m=1}^{\nu}\left(\partial_{\tilde{y}_{m n^{\prime}}^{(12)}}-\partial_{\tilde{y}_{n^{\prime} m}^{(23)}}\right) p_{[3]}^{-}=0, \quad n^{\prime}=2, \ldots, \nu \text {. }
$$

Using the method of characteristic equations we obtain the solution of Eqs.(D.15)

$$
p_{[3]}^{-}=p_{[3]}^{-}\left(B_{n}^{(a)} ; y_{m^{\prime} n^{\prime}}^{(12)}, y_{m^{\prime} n}^{(23)}, y_{m n}^{(31)} ; \alpha_{m n}^{(a a)}\right),
$$

where new variables $y_{m^{\prime} n^{\prime}}^{(12)}$ and $y_{m^{\prime} n}^{(23)}$ are defined by

$$
y_{m^{\prime} n^{\prime}}^{(12)}=\tilde{y}_{m^{\prime} n^{\prime}}^{(12)}-\tilde{y}_{1 n^{\prime}}^{(12)}, \quad y_{m^{\prime} n}^{(23)}=\tilde{y}_{m^{\prime} n}^{(23)}+\tilde{y}_{1 m^{\prime}}^{(12)}, \quad m^{\prime}, n^{\prime}=2, \ldots, \nu .
$$

Thus, the solution of Eqs.(D.5)-(D.7) is given by formula (D.16). Representation of the variables $y_{m^{\prime} n^{\prime}}^{(12)}, y_{m^{\prime} n}^{(23)}, y_{m n}^{(31)}(\underline{\mathrm{D} .16})$ in terms of the generic variables $x_{m n}^{(a b)}(\underline{\mathrm{D} .4})$ is given by

$$
\begin{gathered}
y_{m^{\prime} n^{\prime}}^{(12)}=x_{m^{\prime} n^{\prime}}^{(12)}-x_{m^{\prime} 1}^{(12)}-x_{1 n^{\prime}}^{(12)}+x_{11}^{(12)}, \quad y_{m^{\prime} n}^{(23)}=x_{m^{\prime} n}^{(23)}-x_{1 n}^{(23)}+x_{1 m^{\prime}}^{(12)}-x_{11}^{(12)}, \\
y_{m n}^{(31)}=x_{m n}^{(31)}+x_{n 1}^{(12)}+x_{1 m}^{(23)} .
\end{gathered}
$$

To express $p_{[3]}^{-}(\underline{\mathrm{D} .16})$ in terms of more convenient new independent variables we note the relations

$$
\begin{aligned}
& y_{m^{\prime} n^{\prime}}^{(12)}=x_{m^{\prime} n^{\prime} 1}-x_{m^{\prime} 11}-x_{1 n^{\prime} 1}+x_{111}, \\
& y_{m^{\prime} 1}^{(23)}=x_{1 m^{\prime} 1}-x_{111}, \quad y_{m^{\prime} n^{\prime}}^{(23)}=x_{1 m^{\prime} n^{\prime}}-x_{11 n^{\prime}}, \\
& y_{11}^{(31)}=x_{111}, \quad y_{1 m^{\prime}}^{(31)}=x_{m^{\prime} 11}, \quad y_{m^{\prime} 1}^{(31)}=x_{11 m^{\prime}}, \quad y_{m^{\prime} n^{\prime}}^{(31)}=x_{n^{\prime} 1 m^{\prime}},
\end{aligned}
$$

where the new independent variables

$$
x_{111}, \quad x_{m^{\prime} 11}, \quad x_{1 m^{\prime} 1}, \quad x_{11 m^{\prime}}, \quad x_{1 m^{\prime} n^{\prime}} \quad x_{m^{\prime} 1 n^{\prime}}, \quad x_{m^{\prime} n^{\prime} 1},
$$

are defined by the relations

$$
x_{m n q} \equiv x_{m n}^{(12)}+x_{n q}^{(23)}+x_{q m}^{(31)} .
$$

Note that a number of the independent variables (D.22) is equal to $3 \nu^{2}-3 \nu+1$, while the variables $x_{m n q}$ (D.23) constitute overflow basis of variables. We prefer to exploit overflow basis of the variables (D.23) because this basis is defined symmetrically with respect to all sort of oscillators. The variables ( $(\overline{\mathrm{D} .23})$, being non-polynomial with respect to oscillators, admit the representation

$$
x_{m n q}=\frac{Z_{m n q}}{B_{m}^{(1)} B_{n}^{(2)} B_{q}^{(3)}},
$$


where the cubic forms $Z_{m n q}(5.3)$ are polynomial in oscillators. In view of (D.24), we can use the variables $B_{n}^{(a)}$ and $Z_{m n q}$ in place of $B_{n}^{(a)}$ and $x_{m n q}$. This gives vertex in (5.2).

Derivation of vertex (6.5). Equations to be solved are obtainable from Eqs.(4.48), (4.46) by plugging values of $\mathrm{m}_{a}$ (6.4) in Eq.(4.46). Since the ket-vectors of massless fields (2.12) are independent of the scalar oscillators $\alpha_{n}$, the scalar oscillators $\alpha_{n}^{(1)}, \alpha_{n}^{(2)}$ in (4.53) do not contribute to the Hamiltonian (3.3) and we can therefore ignore dependence on $\alpha_{n}^{(1)}-, \alpha_{n}^{(2)}$-terms in (4.53) 42 . Thus, we start with the vertex and equations

$$
\begin{aligned}
& p_{[3]}^{-}=p_{[3]}^{-}\left(B_{n}^{(a)}, \alpha_{n}^{(3)}, \alpha_{m n}^{(a a+1)}, \alpha_{m n}^{(11)}, \alpha_{m n}^{(22)}, Q_{m n}^{(33)}\right), \\
& \left\{\frac{1}{2} \mathrm{~m}_{3}^{2} \partial_{B_{n}^{(1)}}+\sum_{m=1}^{\nu} B_{m}^{(2)} \partial_{\alpha_{n m}^{(12)}}-\left(B_{m}^{(3)}-\frac{1}{2} \mathrm{~m}_{3} \alpha_{m}^{(3)}\right) \partial_{\alpha_{m n}^{(31)}}\right\} p_{[3]}^{-}=0, \\
& \left\{-\frac{1}{2} \mathrm{~m}_{3}^{2} \partial_{B_{n}^{(2)}}+\sum_{m=1}^{\nu}\left(B_{m}^{(3)}+\frac{1}{2} \mathrm{~m}_{3} \alpha_{m}^{(3)}\right) \partial_{\alpha_{n m}^{(23)}}-B_{m}^{(1)} \partial_{\alpha_{m n}^{(12)}}\right\} p_{[3]}^{-}=0, \\
& \left\{\mathrm{~m}_{3} \partial_{\alpha_{n}^{(3)}}+\sum_{m=1}^{\nu} B_{m}^{(1)} \partial_{\alpha_{n m}^{(31)}}-B_{m}^{(2)} \partial_{\alpha_{m n}^{(23)}}\right\} p_{[3]}^{-}=0 .
\end{aligned}
$$

Introducing in place of $\alpha_{m n}^{(23)}, \alpha_{m n}^{(31)}$ new variables $\widetilde{Q}_{m n}^{(23)}, \widetilde{Q}_{m n}^{(31)}$ defined by

$$
\widetilde{Q}_{m n}^{(23)}=\alpha_{m n}^{(23)}+\frac{\alpha_{n}^{(3)}}{\mathrm{m}_{3}} B_{m}^{(2)}, \quad \widetilde{Q}_{m n}^{(31)}=\alpha_{m n}^{(31)}-\frac{\alpha_{m}^{(3)}}{\mathrm{m}_{3}} B_{n}^{(1)},
$$

we recast vertex (D.25) and Eqs.(D.26)-(D.28) into the form

$$
\begin{aligned}
& p_{[3]}^{-}=p_{[3]}^{-}\left(B_{n}^{(a)}, \alpha_{n}^{(3)}, \alpha_{m n}^{(12)}, \widetilde{Q}_{m n}^{(23)}, \widetilde{Q}_{m n}^{(31)}, \alpha_{m n}^{(11)}, \alpha_{m n}^{(22)}, Q_{m n}^{(33)}\right), \\
& \left\{\frac{1}{2} \mathrm{~m}_{3}^{2} \partial_{B_{n}^{(1)}}+\sum_{m=1}^{\nu} B_{m}^{(2)} \partial_{\alpha_{n m}^{(12)}}-B_{m}^{(3)} \partial_{\widetilde{Q}_{m n}^{(31)}}\right\} p_{[3]}^{-}=0, \\
& \left\{-\frac{1}{2} \mathrm{~m}_{3}^{2} \partial_{B_{n}^{2}}+\sum_{m=1}^{\nu} B_{m}^{(3)} \partial_{\widetilde{Q}_{n m}^{(23)}}-B_{m}^{(1)} \partial_{\alpha_{m n}^{(12)}}\right\} p_{[3]}^{-}=0, \\
& \mathrm{~m}_{3} \partial_{\alpha_{n}^{(3)}} p_{[3]}^{-}=0 .
\end{aligned}
$$

Equations (D.33) tell us that vertex $p_{[3]}^{-}(\overline{\mathrm{D} .30})$ does not depend on $\alpha_{n}^{(3)}$. Keeping this in mind and introducing in place of $\alpha_{m n}^{(12)}, \widetilde{Q}_{m n}^{(23)}, \widetilde{Q}_{m n}^{(31)}$ new variables $Q_{m n}^{(a a+1)}, a=1,2,3$, defined by

$$
\begin{gathered}
Q_{m n}^{(12)}=\alpha_{m n}^{(12)}-\frac{2}{\mathrm{~m}_{3}^{2}} B_{m}^{(1)} B_{n}^{(2)} \\
Q_{m n}^{(23)}=\widetilde{Q}_{m n}^{(23)}+\frac{2}{\mathrm{~m}_{3}^{2}} B_{m}^{(2)} B_{n}^{(3)}, \quad Q_{m n}^{(31)}=\widetilde{Q}_{m n}^{(31)}+\frac{2}{\mathrm{~m}_{3}^{2}} B_{m}^{(3)} B_{n}^{(1)},
\end{gathered}
$$

we recast vertex $p_{[3]}^{-}(\overline{D .30})$ and the remaining Eqs.(D.31), (D.32) into the form

$$
\begin{aligned}
& p_{[3]}^{-}=p_{[3]}^{-}\left(B_{n}^{(a)}, Q_{m n}^{(12)}, Q_{m n}^{(23)}, Q_{m n}^{(31)}, \alpha_{m n}^{(11)}, \alpha_{m n}^{(22)}, Q_{m n}^{(33)}\right), \\
& \mathrm{m}_{3}^{2} \partial_{B_{n}^{(1)}} p_{[3]}^{-}=0, \quad \mathrm{~m}_{3}^{2} \partial_{B_{n}^{(2)}} p_{[3]}^{-}=0 .
\end{aligned}
$$

\footnotetext{
${ }^{42}$ Derivatives with respect to the scalar oscillators $\alpha_{n}^{(1)}, \alpha_{n}^{(2)}$ vanish in 4.48, 4.46) when $\mathrm{m}_{1}=\mathrm{m}_{2}=0$, i.e. ignoring dependence on $\alpha_{n}^{(1)}, \alpha_{n}^{(2)}$ in the cubic interaction vertex is a self-consistent procedure in solving Eq. (4.48).
} 
Equations (D.36) tell us that vertex $p_{[3]}^{-}(\mathrm{D} .35)$ does not depend on $B_{n}^{(1)}$ and $B_{n}^{(2)}$. Keeping this in mind and inserting $\widetilde{Q}_{m n}^{(23)}, \widetilde{Q}_{m n}^{(31)}(\mathrm{D} .29)$ in expressions for $Q_{m n}^{(23)}, Q_{m n}^{(31)}(\mathrm{D} .34)$ we obtain vertex (6.5)- (6.9).

Derivation of vertex (6.57). Equations to be solved are obtainable from Eqs.(4.48), (4.46) by plugging values of $\mathrm{m}_{a}(6.56)$ in Eq.(4.46). Since the ket-vector of massless field (2.12) is independent of the scalar oscillators $\alpha_{n}$, the scalar oscillators in $\alpha_{n}^{(3)}$ (4.53) do not contribute to the Hamiltonian (3.3) and we can therefore ignore dependence on $\alpha_{n}^{(3)}$-terms in (4.53) 43 . Thus, we start with the vertex and equations

$$
\begin{aligned}
& p_{[3]}^{-}=p_{[3]}^{-}\left(B_{n}^{(a)}, \alpha_{n}^{(1)}, \alpha_{n}^{(2)}, \alpha_{m n}^{(a a+1)}, Q_{m n}^{(11)}, Q_{m n}^{(22)}, \alpha_{m n}^{(33)}\right), \\
& \left\{-\frac{1}{2} \mathrm{~m}_{2}^{2} \partial_{B_{n}^{(1)}}+\mathrm{m}_{1} \partial_{\alpha_{n}^{(1)}}+\sum_{m=1}^{\nu}\left(B_{m}^{(2)}+\frac{1}{2} \mathrm{~m}_{2} \alpha_{m}^{(2)}\right) \partial_{\alpha_{n m}^{(12)}}-B_{m}^{(3)} \partial_{\alpha_{m n}^{(31)}}\right\} p_{[3]}^{-}=0, \\
& \left\{\frac{1}{2} \mathrm{~m}_{1}^{2} \partial_{B_{n}^{(2)}}+\mathrm{m}_{2} \partial_{\alpha_{n}^{(2)}}+\sum_{m=1}^{\nu} B_{m}^{(3)} \partial_{\alpha_{n m}^{(23)}}-\left(B_{m}^{(1)}-\frac{1}{2} \mathrm{~m}_{1} \alpha_{m}^{(1)}\right) \partial_{\alpha_{m n}^{(12)}}\right\} p_{[3]}^{-}=0, \\
& \left\{-\frac{\mathrm{m}_{1}^{2}-\mathrm{m}_{2}^{2}}{2} \partial_{B_{n}^{(3)}}+\sum_{m=1}^{\nu}\left(B_{m}^{(1)}+\frac{1}{2} \mathrm{~m}_{1} \alpha_{m}^{(1)}\right) \partial_{\alpha_{n m}^{(31)}}-\left(B_{m}^{(2)}-\frac{1}{2} \mathrm{~m}_{2} \alpha_{m}^{(2)}\right) \partial_{\alpha_{m n}^{(23)}}\right\} p_{[3]}^{-}=0 .
\end{aligned}
$$

Introducing in place of $B_{m}^{(1)}, B_{m}^{(2)}$ the variables $L_{m}^{(1)}, L_{m}^{(2)}$ defined in (6.58) we recast vertex $p_{[3]}^{-}$ (D.37) and Eqs.(D.38)-(D.40) into the form

$$
\begin{aligned}
& p_{[3]}^{-}=p_{[3]}^{-}\left(L_{n}^{(1)}, L_{n}^{(2)}, B_{n}^{(3)}, \alpha_{n}^{(1)} \alpha_{n}^{(2)}, \alpha_{m n}^{(a a+1)}, Q_{m n}^{(11)}, Q_{m n}^{(22)}, \alpha_{m n}^{(33)}\right), \\
& \left\{\mathrm{m}_{1} \partial_{\alpha_{n}^{(1)}}+\sum_{m=1}^{\nu}\left(L_{m}^{(2)}+\frac{\mathrm{m}_{1}^{2}+\mathrm{m}_{2}^{2}}{2 \mathrm{~m}_{2}} \alpha_{m}^{(2)}\right) \partial_{\alpha_{n m}^{(12)}}-B_{m}^{(3)} \partial_{\alpha_{m n}^{(31)}}\right\} p_{[3]}^{-}=0, \\
& \left\{\mathrm{~m}_{2} \partial_{\alpha_{n}^{(2)}}+\sum_{m=1}^{\nu} B_{m}^{(3)} \partial_{\alpha_{n m}^{(23)}}-\left(L_{m}^{(1)}-\frac{\mathrm{m}_{1}^{2}+\mathrm{m}_{2}^{2}}{2 \mathrm{~m}_{1}} \alpha_{m}^{(1)}\right) \partial_{\alpha_{m n}^{(12)}}\right\} p_{[3]}^{-}=0, \\
& \left\{-\frac{\mathrm{m}_{1}^{2}-\mathrm{m}_{2}^{2}}{2} \partial_{B_{n}^{(3)}}+\sum_{m=1}^{\nu}\left(L_{m}^{(1)}+\frac{\mathrm{m}_{1}^{2}-\mathrm{m}_{2}^{2}}{2 \mathrm{~m}_{1}} \alpha_{m}^{(1)}\right) \partial_{\alpha_{n m}^{(31)}}-\left(L_{m}^{(2)}+\frac{\mathrm{m}_{1}^{2}-\mathrm{m}_{2}^{2}}{2 \mathrm{~m}_{2}} \alpha_{m}^{(2)}\right) \partial_{\alpha_{m n}^{(23)}}\right\} p_{[3]}^{-}=0 .
\end{aligned}
$$

Introducing in place of $\alpha_{m n}^{(a a+1)}, a=1,2,3$ new variables $Q_{m n}^{(12)}, \widetilde{Q}_{m n}^{(23)}, \widetilde{Q}_{m n}^{(31)}$ defined by

$$
\begin{aligned}
& Q_{m n}^{(12)}=\alpha_{m n}^{(12)}+\frac{\alpha_{n}^{(2)}}{\mathrm{m}_{2}} L_{m}^{(1)}-\frac{\alpha_{m}^{(1)}}{\mathrm{m}_{1}} L_{n}^{(2)}-\frac{\mathrm{m}_{1}^{2}+\mathrm{m}_{2}^{2}}{2 \mathrm{~m}_{1} \mathrm{~m}_{2}} \alpha_{m}^{(1)} \alpha_{n}^{(2)}, \\
& \widetilde{Q}_{m n}^{(23)}=\alpha_{m n}^{(23)}-\frac{\alpha_{m}^{(2)}}{\mathrm{m}_{2}} B_{n}^{(3)}, \quad \widetilde{Q}_{m n}^{(31)}=\alpha_{m n}^{(31)}+\frac{\alpha_{n}^{(1)}}{\mathrm{m}_{1}} B_{m}^{(3)},
\end{aligned}
$$

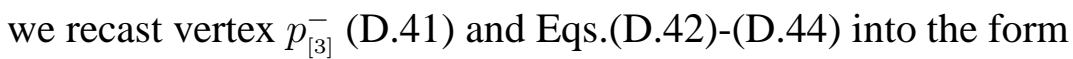

$$
\begin{aligned}
& p_{[3]}^{-}=p_{[3]}^{-}\left(L_{n}^{(1)}, L_{n}^{(2)}, B_{n}^{(3)}, \alpha_{n}^{(1)} \alpha_{n}^{(2)}, Q_{m n}^{(12)}, \widetilde{Q}_{m n}^{(23)}, \widetilde{Q}_{m n}^{(31)}, Q_{m n}^{(11)}, Q_{m n}^{(22)}, \alpha_{m n}^{(33)}\right), \\
& \mathrm{m}_{1} \partial_{\alpha_{n}^{(1)}} p_{[3]}^{-}=0, \quad \mathrm{~m}_{2} \partial_{\alpha_{n}^{(2)}} p_{[3]}^{-}=0, \\
& \left\{-\frac{\mathrm{m}_{1}^{2}-\mathrm{m}_{2}^{2}}{2} \partial_{B_{n}^{(3)}}+\sum_{m=1}^{\nu} L_{m}^{(1)} \partial_{\widetilde{Q}_{n m}^{(31)}}-L_{m}^{(2)} \partial_{\widetilde{Q}_{m n}^{(23)}}\right\} p_{[3]}^{-}=0 .
\end{aligned}
$$

\footnotetext{
${ }^{43}$ Derivatives with respect to the scalar oscillators $\alpha_{n}^{(3)}$ vanish in 4.48, 4.46) when $\mathrm{m}_{3}=0$, i.e. ignoring dependence on $\alpha_{n}^{(3)}$ in the cubic interaction vertex is a self-consistent procedure in solving Eq. (4.48).
} 
Equations (D.47) tell us that vertex $p_{[3]}^{-}(\underline{\mathrm{D} .46})$ does not depend on $\alpha_{n}^{(1)}, \alpha_{n}^{(2)}$. Keeping this in mind and introducing in place of $\widetilde{Q}_{m n}^{(23)}, \widetilde{Q}_{m n}^{(31)}$ the new variables $Q_{m n}^{(23)}, Q_{m n}^{(31)}$ defined by

$$
Q_{m n}^{(23)}=\tilde{Q}_{m n}^{(23)}-\frac{2}{\mathrm{~m}_{1}^{2}-\mathrm{m}_{2}^{2}} B_{n}^{(3)} L_{m}^{(2)}, \quad Q_{m n}^{(31)}=\tilde{Q}_{m n}^{(31)}+\frac{2}{\mathrm{~m}_{1}^{2}-\mathrm{m}_{2}^{2}} B_{m}^{(3)} L_{n}^{(1)},
$$

we recast vertex $p_{[3]}^{-}(\overline{\mathrm{D} .46})$ and the remaining Eqs.(D.48) into the form

$$
\begin{aligned}
& p_{[3]}^{-}=p_{[3]}^{-}\left(L_{n}^{(1)}, L_{n}^{(2)}, B_{n}^{(3)}, Q_{m n}^{(12)}, Q_{m n}^{(23)}, Q_{m n}^{(31)}, Q_{m n}^{(11)}, Q_{m n}^{(22)}, \alpha_{m n}^{(33)}\right), \\
& \left(\mathrm{m}_{1}^{2}-\mathrm{m}_{2}^{2}\right) \partial_{B_{n}^{(3)}} p_{[3]}^{-}=0 .
\end{aligned}
$$

Equations (D.51) tell us that vertex $p_{[3]}^{-}\left(\overline{\mathrm{D} .50)}\right.$ ) does not depend on $B_{n}^{(3)}$. Keeping this in mind and inserting $\widetilde{Q}_{m n}^{(23)}, \widetilde{Q}_{m n}^{(31)}(\mathrm{D} .45)$ in expressions for $Q_{m n}^{(23)}, Q_{m n}^{(31)}(\mathrm{D.49})$ we obtain vertex in (6.57)-(6.63).

Derivation of vertex (6.23). Vertex and appropriate equations to be solved are obtainable from (D.37)-(D.40) by setting $\mathrm{m}_{1}=\mathrm{m}_{2}=\mathrm{m}$. Repeating procedure, we used to solve equations (D.38)-(D.40), we obtain vertex (D.46) and Eqs.(D.47), while Eqs.(D.48) take the form

$$
\sum_{m=1}^{\nu}\left(L_{m}^{(1)} \partial_{\widetilde{Q}_{n m}^{(31)}}-L_{m}^{(2)} \partial_{\widetilde{Q}_{m n}^{(23)}}\right) p_{[3]}^{-}=0 .
$$

The solution of Eqs.(D.47), (D.52) is given by

$$
\begin{aligned}
& p_{[3]}^{-}=p_{[3]}^{-}\left(L_{n}^{(1)}, L_{n}^{(2)}, B_{n}^{(3)}, Q_{m n}^{(12)},, Q_{m n}^{(11)}, Q_{m n}^{(22)}, \alpha_{m n}^{(33)} ; \widetilde{Z}_{m n q}\right), \\
& \widetilde{Z}_{m n q} \equiv L_{m}^{(1)} \widetilde{Q}_{n q}^{(23)}+L_{n}^{(2)} \widetilde{Q}_{q m}^{(31)} .
\end{aligned}
$$

In place of the form $\widetilde{Z}_{m n q}(\mathrm{D.54})$, we prefer to use the form $Z_{m n q}$ defined by

$$
Z_{m n q}=\widetilde{Z}_{m n q}+Q_{m n}^{(12)} B_{q}^{(3)}
$$

Inserting $\widetilde{Z}_{m n q}(\mathrm{D} .54)$ and $\widetilde{Q}_{m n}^{(23)}, \widetilde{Q}_{m n}^{(31)}(\mathrm{D} .45)$ in (D.55) we obtain vertex (6.23)-(6.28).

Derivation of vertex (7.2). Equations to be solved are given in (4.48), (4.46) in which we keep $\mathrm{m}_{a} \neq 0, a=1,2,3$. If we introduce variables $L_{n}^{(a)}(7.3)$ in place of $B_{n}^{(a)}$, then vertex (4.53) and Eqs.(4.48) take the form

$$
\begin{aligned}
p_{[3]}^{-}=p_{[3]}^{-}( & \left.L_{n}^{(a)}, \alpha_{n}^{(a)}, \alpha_{m n}^{(a a+1)}, Q_{m n}^{(a a)}\right), \\
\left\{\mathrm{m}_{a} \partial_{\alpha_{n}^{(a)}}+\right. & \sum_{m=1}^{\nu}\left(L_{m}^{(a+1)}+\frac{\mathrm{m}_{a+1}^{2}+\mathrm{m}_{a}^{2}-\mathrm{m}_{a+2}^{2}}{2 \mathrm{~m}_{a+1}} \alpha_{m}^{(a+1)}\right) \partial_{\alpha_{n m}^{(a a+1)}} \\
& \left.-\left(L_{m}^{(a+2)}-\frac{\mathrm{m}_{a+2}^{2}+\mathrm{m}_{a}^{2}-\mathrm{m}_{a+1}^{2}}{2 \mathrm{~m}_{a+2}} \alpha_{m}^{(a+2)}\right) \partial_{\alpha_{m n}^{(a+2 a)}}\right\} p_{[3]}^{-}=0 .
\end{aligned}
$$

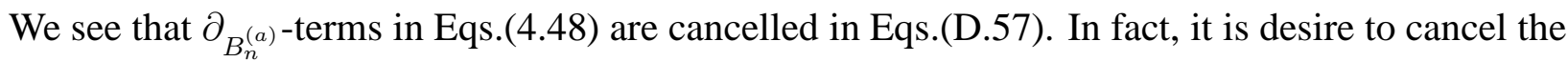
$\partial_{B_{n}^{(a)}}$-terms that motivates usage of the variables $L_{n}^{(a)}$. Now, in place of variables $\alpha_{m n}^{(a a+1)}$ in (D.56), we introduce new variables $Q_{m n}^{(a a+1)}$ defined by

$$
Q_{m n}^{(a a+1)} \equiv \alpha_{m n}^{(a a+1)}+\frac{\alpha_{n}^{(a+1)}}{\mathrm{m}_{a+1}} L_{m}^{(a)}-\frac{\alpha_{m}^{(a)}}{\mathrm{m}_{a}} L_{n}^{(a+1)}+\frac{\mathrm{m}_{a+2}^{2}-\mathrm{m}_{a}^{2}-\mathrm{m}_{a+1}^{2}}{2 \mathrm{~m}_{a} \mathrm{~m}_{a+1}} \alpha_{m}^{(a)} \alpha_{n}^{(a+1)} .
$$


In terms of new variables, vertex $p_{[3]}^{-}(\underline{D .56})$ and Eqs.(D.57) take the form

$$
\begin{aligned}
& p_{[3]}^{-}=p_{[3]}^{-}\left(L_{n}^{(a)}, \alpha_{n}^{(a)}, Q_{m n}^{(a a+1)}, Q_{m n}^{(a a)}\right), \\
& \mathrm{m}_{a} \partial_{\alpha_{n}^{(a)}} p_{[3]}^{-}=0, \quad a=1,2,3 .
\end{aligned}
$$

Because of $\mathrm{m}_{a} \neq 0$ Eqs.(D.60) tell us that vertex $p_{[3]}^{-}\left(\overline{\mathrm{D} .59)}\right.$ does not depend on $\alpha_{n}^{(a)}, a=1,2,3$. Inserting $L_{n}^{(a)}(7.3)$ in (D.58) we obtain vertex $p_{[3]}^{-}$given in (7.2) $-(7.4)$.

\section{Appendix E Derivation of relation (8.19) and Eq.(8.27).}

To derive the representation (8.19) we use $R L, R i$ and $i j$ parts of Eqs.(8.1) given in (8.18). Acting with the angular momentum $\mathbf{J}^{I J}(4.7)$ on vertex $p_{[3]}^{-}(8.16)$, we find the relations

$$
\begin{aligned}
& \mathbf{J}^{R L} p_{[3]}^{-}=\left(\mathbb{P}^{L}\right)^{k} E_{q}\left(\mathbf{M}^{R L}+2 \rho \partial_{\rho}-k\right) \tilde{V}, \\
& \mathbf{J}^{R i} p_{[3]}^{-}=q^{i} \mathbf{J}^{R L} p_{[3]}^{-}+\left(\mathbb{P}^{L}\right)^{k} E_{q}\left(\mathbf{M}^{R i}-\rho \mathbf{M}^{L i}+q^{j} \mathbf{M}^{i j}\right) \widetilde{V}, \\
& \mathbf{J}^{i j} p_{[3]}^{-}=\left(\mathbb{P}^{L}\right)^{k} E_{q} \mathbf{M}^{i j} \widetilde{V} .
\end{aligned}
$$

From these relations, it is easily seen that Eqs.(8.18) amount to the following equations:

$$
\begin{aligned}
& \left(\mathbf{M}^{R L}+2 \rho \partial_{\rho}-k\right) \widetilde{V}=0, \\
& \left(\mathbf{M}^{R i}-\rho \mathbf{M}^{L i}\right) \widetilde{V}=0, \\
& \mathbf{M}^{i j} \tilde{V}=0
\end{aligned}
$$

From relations $(8.8)$ and the fact that the vertex $p_{[3]}^{-}$is degree $k$ homogeneous polynomial in $\mathbb{P}^{I}$, it follows that $\widetilde{V}$ should be degree $k$ polynomial in the momentum $\rho$, i.e. we can use the expansion

$$
\widetilde{V}\left(\rho, \beta_{a} ; \alpha\right)=\sum_{n=0}^{k} \rho^{n} \widetilde{V}_{n}\left(\beta_{a} ; \alpha\right) .
$$

Plugging this expansion in Eq. $($ E.5 $)$ we get the following equations:

$$
\begin{aligned}
& \mathbf{M}^{R i} \widetilde{V}_{n}=\mathbf{M}^{L i} \widetilde{V}_{n-1}, \quad n=1, \ldots, k, \\
& \mathbf{M}^{R i} \widetilde{V}_{0}=0 \\
& \mathbf{M}^{L i} \widetilde{V}_{k}=0
\end{aligned}
$$

while Eqs.(E.4), (E.6) lead to the respective equations for $\widetilde{V}_{0}$ given in (8.21), (8.23). In view of Eq.(E.9), we therefore conclude that $\widetilde{V}_{0}$ should satisfy Eqs.(8.21)-(8.23). Now we focus on Eqs.(E.8), which tell us that $\widetilde{V}_{n}$ can be expressed in terms of $\widetilde{V}_{0}$. Making use of Eqs.(E.8), (E.6) one can make sure that $\widetilde{V}_{n}$ can be presented in the form

$$
\widetilde{V}_{n}=f_{n}\left(\mathbf{M}^{L j} \mathbf{M}^{L j}\right)^{n} \widetilde{V}_{0}, \quad f_{0}=1 .
$$

Now, making use of Eqs.(8.21)-(8.23) and commutators

$$
\left[\mathbf{M}^{R i},\left(\mathbf{M}^{L j} \mathbf{M}^{L j}\right)^{n}\right]=\left(\mathbf{M}^{L j} \mathbf{M}^{L j}\right)^{n-1} 2 n\left(\mathbf{M}^{L j} \mathbf{M}^{j i}-\mathbf{M}^{L i} \mathbf{M}^{R L}-\left(\frac{N^{\prime}}{2}-n\right) \mathbf{M}^{L i}\right)
$$


we get

$$
\mathbf{M}^{R i}\left(\mathbf{M}^{L j} \mathbf{M}^{L j}\right)^{n} \widetilde{V}_{0}=-n\left(N^{\prime}+2 k-2 n\right)\left(\mathbf{M}^{L j} \mathbf{M}^{L j}\right)^{n-1} \mathbf{M}^{L i} \widetilde{V}_{0},
$$

where $N^{\prime} \equiv d-4$. Making use of (E.13), (E.11) in Eqs. (E.8) gives the equations for $f_{n}$,

$$
\frac{f_{n-1}}{f_{n}}=-n\left(N^{\prime}+2 k-2 n\right) \text {. }
$$

The solution of these equations with $f_{0}=1$ is easily found to be

$$
f_{n}=(-)^{n} \frac{\Gamma\left(\frac{N^{\prime}}{2}+k-n\right)}{2^{n} n ! \Gamma\left(\frac{N^{\prime}}{2}+k\right)},
$$

where $\Gamma$ is the Euler gamma function. Collecting all the steps of the derivation given in relations (E.7), (E.11), (E.15) we arrive at the solution given in (8.19), (8.20). Note also that taking into account the solution for $\widetilde{V}_{k}$ and Eq.(E.10) we get the additional equation to be imposed on the vertex $\widetilde{V}_{0}$ :

$$
\mathbf{M}^{L i}\left(\mathbf{M}^{L j} \mathbf{M}^{L j}\right)^{k} \widetilde{V}_{0}=0 .
$$

Our experience led us to a conclusion that Eq.(E.16) is satisfied automatically provided we satisfied all the equations above-discussed. Therefore, we have not displayed Eq.(E.16) in Section 8 ,

Having derived vertex (8.25), we are ready to demonstrate that this vertex satisfies the harmonic equation (8.3). To this end we rewrite operator $\partial_{\mathbb{P}^{I}} \partial_{\mathbb{P}^{I}}$ in terms of the momenta $\mathbb{P}^{L}, q^{i}, \rho(8.8)$

$$
\partial_{\mathbb{P}^{I}} \partial_{\mathbb{P}^{I}}=\left(\mathbb{P}^{L}\right)^{-2}\left(\left(2 \mathbb{P}^{L} \partial_{\mathbb{P}^{L}}+N-4-2 \rho \partial_{\rho}\right) \partial_{\rho}+\partial_{q^{i}} \partial_{q^{i}}\right) .
$$

Acing with $\partial_{\mathbb{P}^{I}} \partial_{\mathbb{P}^{I}}(\underline{\mathrm{E} .17})$ on vertex $(\underline{8.25})$ and using solution for $f_{n}(\underline{\mathrm{E} .15})$, we obtain

$$
\partial_{\mathbb{P}^{I}} \partial_{\mathbb{P}^{I}}\left|p_{[3]}^{-}\right\rangle=\left(\mathbb{P}^{L}\right)^{k-2} E_{q}(-\rho)^{k} f_{k}\left(\mathbf{M}^{L j} \mathbf{M}^{L j}\right)^{k+1} \widetilde{V}_{0} .
$$

From this formula and (E.16), we see that the harmonic equation $(8.3)$ is satisfied indeed.

We now demonstrate that the locality equation (8.26) leads to Eq.(8.27). Making use of notation adopted in (C.3) we rewrite the locality equation (8.26) as

$$
\mathcal{X}_{\text {harm }}^{L}\left|p_{[3]}^{-}\right\rangle=0, \quad \mathcal{X}_{\text {harm }}^{L} \equiv X^{L R} \mathcal{P}^{L}+X^{L i} \mathcal{P}^{i} .
$$

The operator $\mathcal{X}_{\text {harm }}^{L}$, being differential operator in the momentum $\mathbb{P}^{I}$, can be rewritten in terms of the momenta $\mathbb{P}^{L}, q^{i}, \rho(8.8)$. To this end we use chain rules

$$
\begin{aligned}
\mathcal{P}^{L} & =\mathbb{P}^{L}\left(1-\frac{2}{2 \widehat{k}+N-2} \rho \partial_{\rho}\right), \\
\mathcal{P}^{i} & =\mathbb{P}^{L}\left(q^{i}\left(1-\frac{2}{2 \widehat{k}+N-2} \rho \partial_{\rho}\right)-\frac{2}{2 \widehat{k}+N-2} \rho \partial_{q^{i}}\right),
\end{aligned}
$$

where the operator $\widehat{k}(4.33)$ takes the form $\widehat{k}=\mathbb{P}^{L} \partial_{\mathbb{P}^{L}}$. The differential operators $\mathcal{P}^{L}, \mathcal{P}^{i}$ in (E.19) are acting on the vertex that is degree $k$ monomial in $\mathbb{P}^{L}$ (8.33). Therefore in expressions for $\mathcal{P}^{L}$, $\mathcal{P}^{i}($ E.20), (E.21) we can use the identification $\widehat{k}=k$. By using this identification and expressions for $X^{I J}$ (4.19) we recast the operator $\mathcal{X}_{\text {harm }}^{L}$ into the form

$$
\mathcal{X}_{\text {harm }}^{L}=\sum_{a=1}^{3} \check{\beta}_{a}\left(\beta_{a} \partial_{\beta_{a}}+M^{(a) R L}-q^{i} M^{(a) L i}\right) \mathcal{P}^{L}+\frac{2 \mathbb{P}^{L} \rho}{2 k+N-2} \check{\beta}_{a} M^{(a) L i} \partial_{q^{i}}
$$


Acting with this operator on vertex $\left|p_{[3]}^{-}\right\rangle(\underline{8.25})$, we obtain sequence of the relations

$$
\begin{aligned}
\mathcal{X}_{\text {harm }}^{L}\left|p_{[3]}^{-}\right\rangle & =\left(\mathbb{P}^{L}\right)^{k} \widehat{E}_{q} \sum_{a=1}^{3}\left(\check{\beta}_{a}\left(\beta_{a} \partial_{\beta_{a}}+M^{(a) R L}\right) \mathcal{P}^{L}-\frac{2 \mathbb{P}^{L} \rho \check{\beta}_{a} M^{(a) L i}}{2 k+N-2} \mathbf{M}^{L i}\right) \widehat{E}_{\rho} \widetilde{V}_{0} \\
& =\left(\mathbb{P}^{L}\right)^{k} \widehat{E}_{q} \widehat{E}_{\rho} \mathcal{P}^{L} \sum_{a=1}^{3} \check{\beta}_{a}\left(\beta_{a} \partial_{\beta_{a}}+M^{(a)} R L\right) \widetilde{V}_{0} \\
& -\frac{2\left(\mathbb{P}^{L} \rho\right)^{k+1} \widehat{E}_{q}}{2 k+N-2} f_{k} \sum_{a=1}^{3} \check{\beta}_{a} M^{(a) L i} \mathbf{M}^{L i}\left(\mathbf{M}^{L j} \mathbf{M}^{L j}\right)^{k} \widetilde{V}_{0}
\end{aligned}
$$

where $f_{k}$ is given in (E.15). From the 2 nd line of relations $(\overline{E .23})$ we see that Eq. (8.26) (or (E.19) gives the desired Eq.(8.27). Expression in the 3rd line in (E.23) is equal to zero due to Eq.(E.16). 


\section{References}

[1] P. A. M. Dirac, Rev. Mod. Phys. 21, 392 (1949).

[2] S. Weinberg, Phys. Rev. 150, 1313 (1966).

[3] J. B. Kogut and D. E. Soper, Phys. Rev. D 1, 2901 (1970).

[4] P. Goddard, J. Goldstone, C. Rebbi and C. B. Thorn, Nucl. Phys. B 56, 109 (1973).

[5] M. Kaku and K. Kikkawa, Phys. Rev. D 10, 1110 (1974).

[6] J. F. L. Hopkinson, R. W. Tucker and P. A. Collins, Phys. Rev. D 12, 1653 (1975).

[7] M. B. Green and J. H. Schwarz, Nucl. Phys. B 218, 43 (1983).

[8] M. B. Green, J. H. Schwarz and L. Brink, Nucl. Phys. B 219, 437 (1983).

[9] M. B. Green and J. H. Schwarz, Nucl. Phys. B 243, 475 (1984).

[10] L. Brink, O. Lindgren and B. E. W. Nilsson, Nucl. Phys. B 212, 401 (1983).

[11] S. Mandelstam, Nucl. Phys. B 213, 149 (1983).

[12] L. Brink and A. Tollsten, Nucl. Phys. B 249, 244 (1985).

[13] M. B. Green and J. H. Schwarz, Phys. Lett. B 122, 143 (1983).

[14] L. Brink, M. B. Green and J. H. Schwarz, Nucl. Phys. B 223, 125 (1983).

[15] H.Hata, K.Itoh, T.Kugo, H.Kunitomo and K.Ogawa, Phys. Rev. D 34, 2360 (1986).

[16] H.Hata, K.Itoh, T.Kugo, H.Kunitomo and K.Ogawa, Phys. Rev. D 35, 1318 (1987).

[17] W. Siegel and B. Zwiebach, Nucl. Phys. B 282, 125 (1987).

[18] W. Siegel and B. Zwiebach, Phys. Lett. B 184, 325 (1987).

[19] W. Siegel, "Introduction To String Field Theory," arXiv:hep-th/0107094.

[20] A. K. H. Bengtsson, I. Bengtsson and L. Brink, Nucl. Phys. B 227, 31 (1983).

[21] A. K. H. Bengtsson, I. Bengtsson and L. Brink, Nucl. Phys. B 227, 41 (1983).

[22] A. K. H. Bengtsson, I. Bengtsson and N. Linden, Class. Quant. Grav. 4, 1333 (1987).

[23] E. S. Fradkin and R. R. Metsaev, Class. Quant. Grav. 8, L89 (1991).

[24] S.J.Brodsky, H.C.Pauli and S.S.Pinsky, Phys.Rept. 301, 299 (1998) hep-ph/9705477

[25] B. de Wit, J. Hoppe and H. Nicolai, Nucl. Phys. B 305, 545 (1988).

[26] E. Bergshoeff, E. Sezgin, Y. Tanii and P. K. Townsend, Annals Phys. 199, 340 (1990).

[27] O. Bergman and C. B. Thorn, Phys. Rev. D 52, 5980 (1995) [arXiv:hep-th/9506125].

[28] E. S. Fradkin and M. A. Vasiliev, Phys. Lett. B 189, 89 (1987).

[29] M. A. Vasiliev, Phys. Lett. B 243, 378 (1990).

[30] M. A. Vasiliev, Phys. Lett. B 567, 139 (2003) [arXiv:hep-th/0304049].

[31] M. A. Vasiliev, Comptes Rendus Physique 5, 1101 (2004) [arXiv:hep-th/0409260].

[32] X. Bekaert, S. Cnockaert, C. Iazeolla and M. A. Vasiliev, arXiv:hep-th/0503128.

[33] M. A. Vasiliev, Sakharov memorial lectures in physics, vol.1, 427-445; Moscow 1991

[34] R. R. Metsaev, Nucl. Phys. B 563, 295 (1999) [arXiv:hep-th/9906217].

[35] R. R. Metsaev, Phys. Lett. B 531, 152 (2002) [arXiv:hep-th/0201226].

[36] R. R. Metsaev, Phys. Lett. B 590, 95 (2004) [arXiv:hep-th/0312297].

[37] F. A. Berends, G. J. H. Burgers and H. Van Dam, Z. Phys. C 24, 247 (1984). 
[38] F. A. Berends, G. J. H. Burgers and H. van Dam, Nucl. Phys. B 260, 295 (1985).

[39] X. Bekaert, N. Boulanger and S. Cnockaert, JHEP 0601, 052 (2006) arXiv:hep-th/0508048].

[40] N. Boulanger, S. Leclercq and S. Cnockaert, Phys. Rev. D 73, 065019 (2006) [arXiv:hep-th/0509118].

[41] S. Deser and Z. Yang, Class. Quant. Grav. 7, 1491 (1990).

[42] F. A. Berends, G. J. H. Burgers and H. van Dam, Nucl. Phys. B 271, 429 (1986).

[43] S. Weinberg, Phys. Rev. 181, 1893 (1969).

[44] S. Weinberg, Phys. Rev. 133, B1318 (1964).

[45] S. Weinberg, Phys. Rev. 134, B882 (1964).

[46] T. Hurth and K. Skenderis, Nucl. Phys. B 541, 566 (1999) [arXiv:hep-th/9803030].

[47] J. E. Paton and H. M. Chan, Nucl. Phys. B 10, 516 (1969).

[48] R. R. Metsaev, Mod. Phys. Lett. A 6, 2411 (1991).

[49] M. Goroff and J. H. Schwarz, Phys. Lett. B 127, 61 (1983).

[50] T. Hori, Nucl. Phys. B 274, 401 (1986).

[51] C. Aragone and A. Khoudeir, Class. Quant. Grav. 7, 1291 (1990).

[52] A. A. Tseytlin, Phys. Lett. B 185, 59 (1987).

[53] R. R. Metsaev, Class. Quant. Grav. 10, L39 (1993).

[54] R. R. Metsaev, Phys. Lett. B 309, 39 (1993).

[55] R. R. Metsaev, Mod. Phys. Lett. A 8, 2413 (1993).

[56] R. R. Metsaev and A. A. Tseytlin, Phys. Lett. B 185, 52 (1987).

[57] S. N. Gupta and W. W. Repko, Phys. Rev. 165, 1415 (1968)

[58] S. Ferrara, M. Porrati and V. L. Telegdi, Phys. Rev. D 46, 3529 (1992).

[59] R. R. Metsaev, Phys. Rev. D 71, 085017 (2005) [arXiv:hep-th/0410239].

[60] S.M.Klishevich and Y.M.Zinovev, Phys.Atom.Nucl. 61, 1527 (1998); hep-th/9708150.

[61] S. M. Klishevich, Int. J. Mod. Phys. A 15, 535 (2000) [arXiv:hep-th/9810228].

[62] P. C. Argyres and C. R. Nappi, Phys. Lett. B 224, 89 (1989).

[63] S. M. Klishevich, Int. J. Mod. Phys. A 15, 395 (2000) [arXiv:hep-th/9805174].

[64] A. Cucchieri, M. Porrati and S. Deser, Phys. Rev. D 51, 4543 (1995) [arXiv:hep-th/9408073].

[65] S. M. Klishevich, Class. Quant. Grav. 16, 2915 (1999) [arXiv:hep-th/9812005].

[66] I. L. Buchbinder, D. M. Gitman, V. A. Krykhtin and V. D. Pershin, Nucl. Phys. B 584, 615 (2000) [arXiv:hep-th/9910188].

[67] I. L. Buchbinder, D. M. Gitman and V. D. Pershin, Phys. Lett. B 492, 161 (2000) hep-th/0006144

[68] N. Boulanger and L. Gualtieri, Class. Quant. Grav. 18, 1485 (2001) [arXiv:hep-th/0012003].

[69] X. Bekaert, N. Boulanger and S. Cnockaert, J. Math. Phys. 46, 012303 (2005) [arXiv:hep-th/0407102].

[70] T. Pengpan and P. Ramond, Phys. Rept. 315, 137 (1999) [arXiv:hep-th/9808190].

[71] L. Brink, P. Ramond and X. z. Xiong, JHEP 0210, 058 (2002) [hep-th/0207253].

[72] L. Brink, "Euler multiplets and light-cone supersymmetry," arXiv:hep-th/0203008

[73] L. Brink and P. Ramond, "Dirac equations, light cone supersymmetry, and superconformal algebras," arXiv:hep-th/9908208. 